MASSACHUSETTS INSTITUTE OF TECHNOLOGY METEOROLOGICAL PAPERS, VOL. I, NO. 3

\title{
THERMODYNAMICS APPLIED TO AIR MASS ANALYSIS
}

\author{
BY \\ C.-G. ROSSBY
}

CAMBRIDGE, MASSACHUSETTS

1932 


\section{INTRODUCTION}

CINCE the beginning of 1929 systematic work has been carried on at the Massachusetts $\checkmark$ Institute of Technology to develop practical methods for the identification and characterization of air masses with the aid of upper air soundings of pressure, temperature and humidity. A brief report on this work was published in October 1930'. It was then shown that by plotting against each other two meteorological elements, which under certain well defined conditions are recognized as conservative, namely, specific humidity and potential temperature, curves are obtained which, in winter time, to a high extent remain unchanged and characteristic of the individual air masses. In view of this property the curves were named "invariant curves." Since the invariance is restricted to the winter season, but the curves always may be advantageously used to determine the vertical structure and life history of air masses, they shall, in the following, be referred to as "characteristic curves."

The report also stated that by means of characteristic curves a new method had been created of indicating certain differences in stability between the principal American air masses. These differences may be expressed in terms of the variation with elevation of specific entropy, and it was therefore decided to continue the investigation and to include in it a study of the equivalent-potential temperature, which, in an easily comprehensible form, measures the specific entropy of moist air. The excellent results obtained by Robitzsch ${ }^{2}$ through the introduction of equivalent-potential temperature into practical meteorological work lent additional support to this decision.

Through the courtesy of the Chief of the Weather Bureau, Professor C. F. Marvin, we obtained photostat copies of the meteorograph records for the periods October 1, 1929 to March 31, 1930 and July 1 to August 31, 1930 from the five Weather Bureau kite stations, Ellendale, Broken Arrow, Groesbeck, Royal Center, and Due West. In addition, the Navy Department placed at our disposal upper air data from the naval air stations at San Diego, Seattle and Pensacola for parts of the periods referred to above. In all about thirteen hundred soundings were obtained for the investigation. To supplement these records with observations from New England, the Meteorological Department of the Massachusetts Institute of Technology is now (since November 16, 1931) making regular airplane soundings at Boston and the material thus collected will be included in the final analysis.

To facilitate the discussion of these data, tables for the calculation of the equivalentpotential temperature were prepared. Since potential temperatures and specific humidities (or mixing ratios) have to be calculated for the construction of characteristic curves, it was decided to express the equivalent-potential temperature in terms of these two arguments.

Everybody connected with the meteorological course as instructor, assistant or graduate student during the winter 1930-1931 has taken some part in the calculation of the tables for the equivalent-potential temperature or in the evaluation of the kite

1 Earl, K. and Turner, T. A., A Graphical Means of Identifying Airmasses, Massachusetts Institute of Technology Professional Notes, No. 4, Cambridge, Mass., 1930.

2 Robitzsch, M., Die Verwertung der durch aerologische Versuche gewonnenen Feuchtigkeitsdaten zur Diagnose der jeweiligen atmosfärischen Zustände, Die Arbeiten des Preussischen Aeronautischen Observatoriums bei Lindenberg, XVI. Band, Wissenschaftliche Abhandlungen, Heft C, Braunschweig, 1928. 
and airplane records. The final analysis of the upper air data, including a study of the properties of the principal American air masses; is being undertaken by Dr. H. C. Willett. The object of the present communication is primarily to develop certain advantageous methods of attack of air mass problems and only incidentally to present results concerning the structure of American air masses. Specifically, the purpose is threefold:

1. To present simple, consistent definitions of the equivalent-potential temperature and the equivalent temperature and to discuss theoretically their most important properties as well as the general properties of the characteristic curve.

2. To illustrate by examples the use of characteristic curves and equivalent-potential temperature diagrams in the analysis of air masses.

3. To present tables and diagrams for the equivalent-potential temperature in terms of potential temperature and mixing ratio.

For the sake of those readers who are unwilling to wade through lengthy mathematical derivations before arriving at some practical conclusions, mathematical theory and applications are kept apart in the following presentation. Thus the third section contains a non-mathematical discussion of selected characteristic curves in conjunction with a study of the corresponding synoptic charts. Section one is devoted to theoretical definitions. In this section not all the results are new, but the definitions given are more rigid and consistent than those usually presented. Section two is devoted to the theoretical solution of a problem of atmospheric convection with the aid of equivalent-potential temperatures. Section four consists entirely of tables, diagrams and plates.

I am obliged to Lt. T. J. Raftery, U.S.N., and to Lt. W. M. Graesser, U.S.N., who calculated part of the data discussed in section three. Their results were originally presented in an (unpublished) thesis on file at the Massachusetts Institute of Technology.

Cambridge, Massachusetts

December, 1931 


\section{LIST OF SYMBOLS}

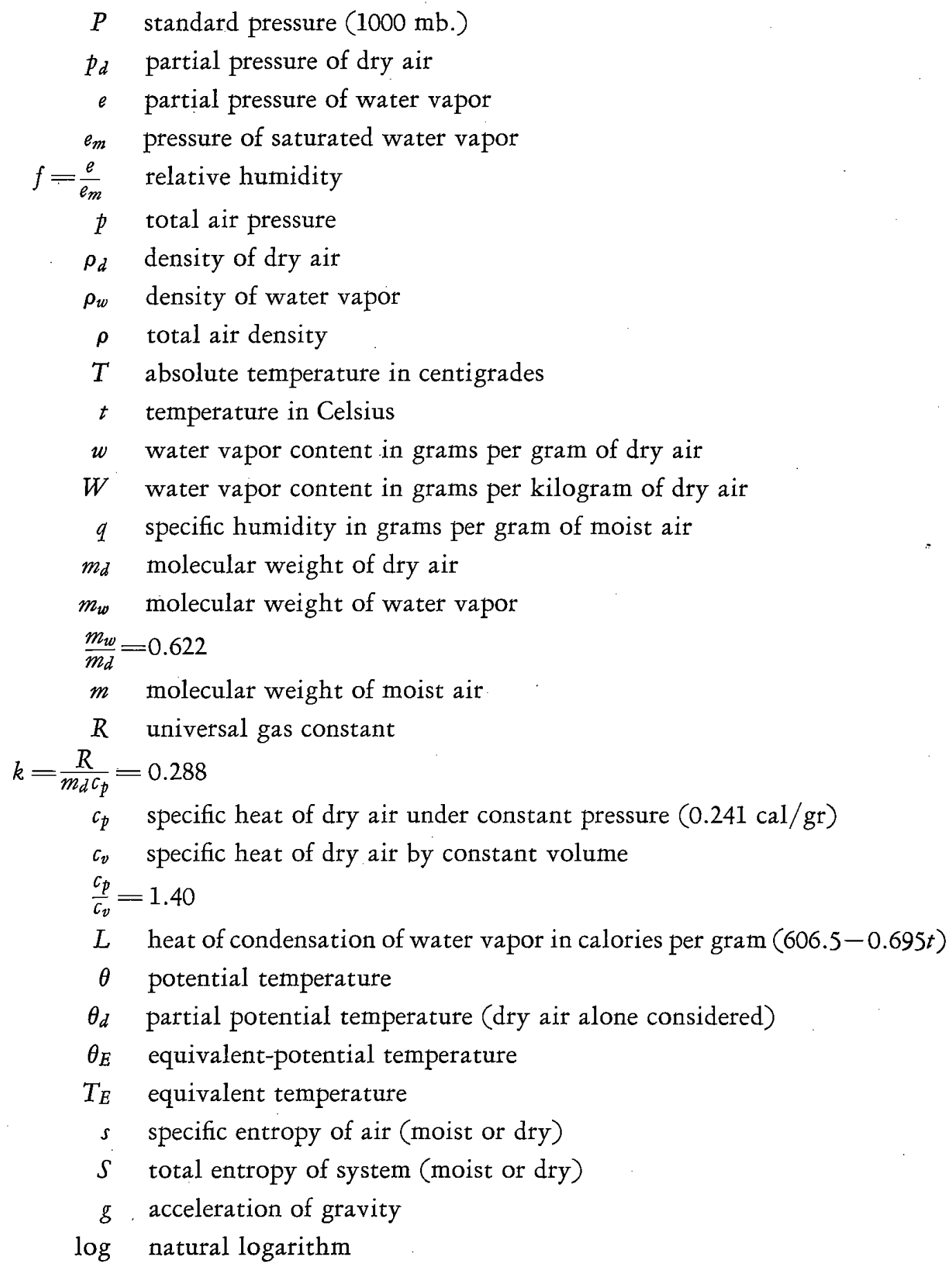





\section{Chapter I}

\section{THEORY OF THE EQUIVALENT-POTENTIAL TEMPERATURE AND THE EQUIVALENT-POTENTIAL TEMPERATURE DIAGRAM}

$T^{\mathrm{N}}$ N THE atmosphere a small, initially unsaturated element is given, containing one gram of dry air and $w$ grams of water vapor. This element is permitted to expand under gradually decreasing pressure. Due to mechanical cooling, part of the water vapor will condense. It is assumed that the liquid water drops out of the element immediately upon condensation. Throughout the process, no heat is added, and no heat leaves the element except as contained in the precipitating water; this latter amount of heat is supposed to be negligibly small. On account of the prevalence of subcooled water in the atmosphere we may assume that the condensation takes place in the form of liquid water even below the freezing point; thus the heat of fusion is not available to raise the temperature of the air. Hence the process outlined follows the "pseudo-adiabatic" rain stage curve.

We then define the equivalent-potential temperature as the ultimate potential temperature assumed by the element when all its water vapor has been removed. This value of the potential temperature is approached asymptotically as the pressure in the element decreases toward zero.

\section{WATER VAPOR CONTENT}

To express our definition analytically we must first review the definitions of some auxiliary quantities. The water vapor content or mixing ratio (w) is expressed in terms of the number of grams of water vapor associated with one gram of dry air. Assuming. the perfect gas law to hold for dry air and water vapor alike, we have, by definition,

$$
w=\frac{\rho_{w}}{\rho_{d}}=\frac{m_{w}}{m_{d}} \cdot \frac{e}{p_{d}}=0.622 \cdot \frac{e_{m}}{p_{d}} \cdot f
$$

or

$$
w=w_{m} \cdot f, \quad\left(f=\frac{e}{e_{m}}\right)
$$

$w_{m}$ signifying the maximum water vapor content possible at the given pressure and temperature, $f$ being the relative humidity. The quantity $w$ is closely related to, but not the same as, the specific humidity $q$, which measures the amount of vapor associated with one gram of moist air. Thus,

$$
q=\frac{\rho_{w}}{\rho_{w}+\rho_{d}}=\frac{w}{1+w} .
$$

The value of $w$ seldom exceeds 0.02 (20 grams of water per kilogram of dry air). Considering the inaccuracy of our humidity readings, we are therefore generally permitted to neglect $w$ in the denominator. Hence approximately,

$$
q=w
$$




\section{Potential and Partial Potential Temperature}

The potential temperature $\theta$ is defined by Poisson's equation

$$
\frac{T}{\theta}=\left(\frac{p}{P}\right)^{k} . \quad\left(k=\frac{R}{m_{d} c_{p}}\right)
$$

\section{*}

The exponent $k$ should be corrected for the presence of water vapor but the correction is insignificant. It is possible and, for our purpose, preferable to introduce another potential temperature $\theta_{d}$, which we may name the partial potential temperature since it is calculated from the partial pressure of dry air. It is defined by

$$
\frac{T}{\theta_{d}}=\left(\frac{p_{d}}{P}\right)^{k}
$$

Since

$$
p=p_{d}+e
$$

it follows that

$$
\frac{T}{\theta}=\left(\frac{p_{d}+e}{P}\right)^{k}
$$

or

$$
\frac{T}{\theta}=\left(\frac{p_{d}}{P}\right)^{k}\left(1+\frac{e}{p_{d}}\right)^{k}
$$

If this last equation is combined with $(1)$ and $(6)$, one obtains

$$
\frac{T}{\theta}=\frac{T}{\theta_{d}}\left(1+\frac{m_{d}}{m_{w}} w\right)^{k}
$$

or

$$
\theta_{d}=\theta\left(1+\frac{m_{d}}{m_{w}} w\right)^{k}
$$

which latter equation for all practical purposes may be written

$$
\theta_{d}=\theta\left(1+k \frac{m_{d}}{m_{w}} w\right)=\theta\left(1+\frac{R}{m_{w} c_{p}} w\right)
$$

As long as the element remains unsaturated $\theta$ and $w$, and therefore also $\theta_{d}$, will remain unaffected by adiabatic changes of state. To determine $\theta_{d}$ from $\theta$ it is necessary to add a correction term

$$
\Delta \theta_{d}=\theta\left[\left(1+\frac{m_{d}}{m_{w}} w\right)^{k}-1\right]
$$

This correction term is tabulated in Table B. When the potential temperature is deter- . mined graphically from the adiabatic chart, the partial potential temperature may be obtained directly by entering the chart with the partial pressure $p_{d}$ and the temperature $T$ as arguments.

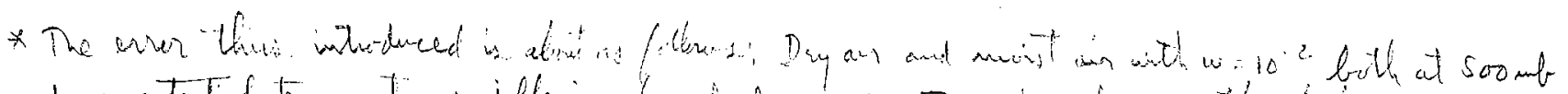

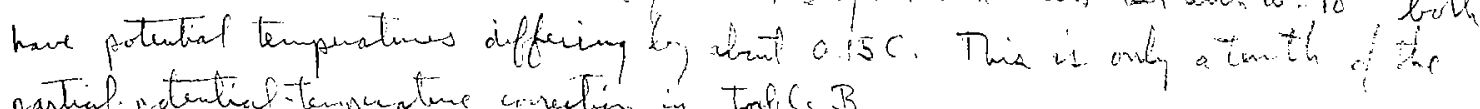

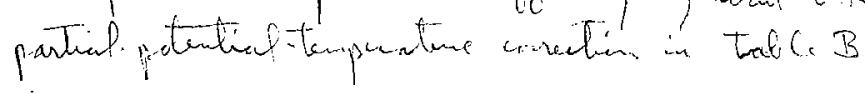




\section{Temperature at Condensation Level}

The temperature at which an adiabatically expanded air particle reaches the condensation point must be determined in order to permit the calculation of the equivalentpotential temperature. Initially the element has the partial pressure $p_{d}$, the temperature $T$ and the moisture content $w$. At the condensation level the corresponding quantities are $p_{o d}, T_{o}$ and $w$, the moisture content $w$ and the partial potential temperature $\theta_{d}$ remaining unchanged up to this point. From Poisson's equation it then follows that

$$
{\frac{p_{o d}}{T_{o}}}^{k}={\frac{p_{d}}{T}}^{k}={\frac{P}{\theta_{d}}}^{k} .
$$

At the condensation level,

$$
w=\frac{m_{w}}{m_{d}} \cdot \frac{e_{o m}}{p_{o d}}
$$

where $e_{o m}$ represents the saturation vapor pressure at the temperature $T_{0}$. Thus

$$
p_{o d}=\frac{m_{w}}{m_{d}} \cdot \frac{e_{o m}}{w}
$$

and

$$
\frac{\left(\frac{m_{w}}{m_{d}} \cdot \frac{e_{o m}}{w}\right)^{k}}{T_{o}}=\frac{P^{k}}{\theta_{d}}
$$

or

$$
\left(\frac{m_{w}}{m_{d} P}\right)^{k} \cdot{\frac{e_{o m}}{T_{o}}}^{k}={\frac{w^{k}}{\theta_{d}}}^{k}
$$

The left side of this equation we have plotted as a function of $T_{0}$. For $e_{o m}$ the values given by Robitzsch ${ }^{3}$ were used (Table A). Since these values are given in millimeters instead of millibars and since $P$ is equal to $1000 \mathrm{mb}$, equation (18) may be written

$$
\left(\frac{4}{3} \frac{m_{w}}{m_{d}}\right)^{k} \cdot \frac{e_{o m}^{k}}{T_{0}}=\frac{W^{k}}{\theta_{d}}, \quad\left(e_{o m} \text { in mm } H g\right)
$$

where $W$ represents the number of grams of water vapor associated with one kilogram of dry air. Equation (19) may then be solved graphically. For the sake of convenience both members may be multiplied by $10^{k}, 100^{k}$ or $1000^{k}$ and the values of the quantities $N^{k}$ taken from a set of tables calculated by T. H. Doerr. ${ }^{4}$

${ }^{3}$ Robitzsch, op. cit. in fn. 2.

4 Doerr, T. N., Tables of 0.288th Powers, Quarterly Journal of the Royal Meteorological Society, Vol. 47, pp. 196-202, London, 1921. 


\section{The Equivalent-Potential Temperature}

It is now easy to determine the equivalent-potential temperature. The characteristic differential equation of the pseudo-adiabatic rain stage 5 is, in our notations,

$$
c_{p} \frac{d T}{T}-\frac{R}{m_{d}} \frac{d p_{d}}{p_{d}}+d\left(\frac{L w}{T}\right)=0
$$

We may at once integrate the above equation. Thus

$$
c_{p} \log T-\frac{R}{m_{d}} \log p_{d}+\frac{L w}{T}=C . \quad(C=\text { constant })
$$

From the definition of the partial potential temperature ( 6 ) we find

$$
c_{p} \log \theta_{d}-c_{p} \log T=\frac{R}{m_{d}} \log P-\frac{R}{m_{d}} \log p_{d}
$$

The characteristic equation may therefore be written

$$
c_{p} \log \theta_{d}-\frac{R}{m_{d}} \log P+\frac{L w}{T}=C
$$

As the pressure decreases towards zero and the water drops out, the potential temperature of the element asymptotically approaches the equivalent-potential temperature. Thus

$$
c_{p} \log \theta_{E}-\frac{R}{m_{d}} \log P=C
$$

and, consequently,

$$
c_{p} \log \theta_{E}=c_{p} \log \theta_{d}+\frac{L w}{T}
$$

or

$$
\theta_{E}=\theta_{d} \mathrm{e}^{\frac{L w}{c_{p} T}} \quad(\mathrm{e}=\text { base of natural logarithms })
$$

for any point on the pseudo-adiabatic curve. Obviously $\theta_{E}$ is constant along a pseudoadiabatic line.

If the element is unsaturated, having a partial potential temperature $\theta_{d}$ and a vapor content $w$, its equivalent-potential temperature is evidently obtained from

$$
\theta_{E}=\theta_{d} \mathrm{e}^{\frac{L_{o} w}{c_{p} T_{o}}}
$$

where $L_{o}$, the heat of condensation, is taken at the temperature $T_{o}$. The latter quantity represents the temperature at the condensation level; it depends upon $w$ and $\theta_{d}$ and may be calculated by means of (19). Thus, to obtain the equivalent-potential temperature

${ }^{5}$ Humphreys, W. J., Physics of the Air, Second Edition, p. 257, McGraw-Hill Book Company, New York and London, 1929. The equation given by Humphreys differs slightly from the exact form derived by J. Fjeldstad, but the discrepancy between the two is small. Humphreys' formula has the advantage of greater simplicity. Fjeldstad's equation is given in Geofysiske Publikasjoner, Vol. III, No. 13, Oslo, 1925. 
from the partial potential temperature a correction term $\Delta \theta_{E}$ has to be added which is given by

$$
\Delta \theta_{E}=\theta_{d}\left(\mathrm{e}^{\frac{L_{o} w}{c_{p} T_{o}}}-1\right)
$$

$\Delta \theta_{E}$ is tabulated in Table C. It was mentioned above that $L_{0}$, the heat of condensation, varies with the temperature $T_{o}$. This variation ${ }^{6}$ may be expressed in the form

$$
L_{o}=606.5-0.695\left(T_{o}-273\right) \text {. }
$$

In calculating $\Delta \theta_{E}$ for different values of $w$ and $\theta_{d}$ we combined (19) and (29) and determined graphically the value of $\frac{L_{o}}{c_{p} T_{o}}$ for different values of $w$ and $\theta_{d}$.

\section{Equivalent Temperature}

The equivalent temperature $T_{E}$ we shall here define as the temperature which a particle of air would assume if it were lifted pseudo-adiabatically until all its moisture had been removed and then were brought back dry-adiabatically to its original dry air pressure. From this definition it follows that

$$
\frac{T_{E}}{\theta_{E}}=\left(\frac{p_{d}}{P}\right)^{k}=\frac{T}{\theta_{d}}
$$

or

$$
T_{E}=T \cdot \frac{\theta_{E}}{\theta_{d}}
$$

Thus the equivalent temperature stands in the same relation to the equivalent-potential temperature as the ordinary temperature to the partial potential temperature. The product of $c_{p}$ in the difference between the equivalent temperature and the actual temperature,

$$
\Delta T_{E}=T\left(\mathrm{e}^{\frac{L_{o} w}{c_{p} T_{o}}}-1\right),
$$

may in a way be said to measure the latent heat of the water vapor originally present in the air. The quantity $\Delta T_{E}$ we shall call the equivalent temperature difference. It is well to notice that the equivalent temperature difference defined above is a function not only of the temperature and the water vapor content but also of the pressure; analytically this is apparent from the fact that a change in pressure affects a change in the temperature $T_{0}$ at which condensation is reached.

It is readily seen that the equivalent temperature difference may be written in the form

$$
\Delta T_{E}=\frac{T}{\theta_{d}} \cdot \Delta \theta_{E}
$$

and calculated from Table C. Frequently the moisture content of the air, and therefore the exponent in (32) is so small that it is permissible to write

$$
\Delta T_{E}=\frac{T}{T_{o}} \frac{L_{o} w}{c_{p}}
$$

\footnotetext{
${ }^{6}$ Robitzsch, op. cit. in fn. 2.
} 
The expressions for the equivalent temperature difference given in (33) and (34) suffer from the disadvantage that they depend upon three variables, $w, T$ and $p_{d}$ (or $f$, the relative humidity). However, the ratio $\frac{T}{T_{0}}$ will be close to unity if the relative humidity of the air particle is high. Then (34) reduces to

$$
\Delta T_{E}=\frac{L_{o} w}{c_{p}}, \quad\left(\therefore T_{E}=T+\frac{L_{o} w}{c_{p}}\right)
$$

which expression, if the slight variation of the heat of condensation $\left(L_{0}\right)$ with temperature be disregarded, depends on one variable only, namely, the water vapor content $w$. The approximate expression (35) is identical with the definition given by Robitzsch ${ }^{7}$ for the equivalent temperature difference. The advantage of the strict definitions of equivalentpotential temperature and equivalent temperature set forth in the preceding discussion may be found in the fact that they are based upon well-defined processes by means of which either of these temperatures may be realized.

\begin{tabular}{|c|c|c|c|c|c|c|c|}
\hline & & $=10 g r$ & $t=+$ & $=289$ & $e_{m}=18.174 \mathrm{mb}$ & & \\
\hline$p_{d}$ & $f \%$ & $\theta_{d}$ & $\theta_{E}$ & $T_{o}$ & $\Delta_{1}=T\left(e^{\frac{L_{o w}}{C_{p} T_{0}}}-1\right)$ & $\Delta_{2}=\frac{T}{T_{0}} \frac{L_{0} w}{c_{p}}$ & $\Delta_{3}=\frac{L u}{c_{p}}$ \\
\hline 1000 & 88 & 289.0 & 315.1 & 287 & 26.1 & 25.0 & 25.2 \\
\hline 900 & 80 & 297.9 & 325.0 & 285 & 26.3 & 25.2 & 25.2 \\
\hline 800 & 71 & 308.2 & 336.6 & 283 & 26.6 & 25.5 & 25.2 \\
\hline 700 & 62 & 320.3 & 350.1 & 280 & 26.9 & 25.7 & 25.2 \\
\hline
\end{tabular}

TABLE 1

TABLE 2

\begin{tabular}{c|c|c|c|c|c|c|c}
\multicolumn{9}{c}{$w=10 \mathrm{gr} / \mathrm{kg}$} & $t=+26^{\circ} \mathrm{C}=299^{\circ} \mathrm{A}$ & $\left(e_{m}=33.604 \mathrm{mb}.\right)$ \\
\hline 1000 & 48 & 299.0 & 326.3 & 284 & 27.3 & 26.1 & 25.2 \\
\hline 900 & 43 & 308.2 & 336.6 & 282 & 27.6 & 26.3 & 25.2 \\
\hline 800 & 38 & 318.8 & 348.5 & 280 & 27.8 & 26.6 & 25.2 \\
\hline 700 & 33 & 331.3 & 362.6 & 278 & 28.2 & 26.9 & 25.2 \\
\hline \hline
\end{tabular}

TABLE 3

$w=5 g r / \mathrm{kg} \quad t=+26^{\circ} \mathrm{C}=299^{\circ} \mathrm{A} \quad\left(e_{n}=33.604 \mathrm{mb}.\right)$

\begin{tabular}{l|l|l|l|l||c|c|c}
\hline 1000 & 24 & 299.0 & 313.1 & 272 & 14.1 & 13.8 & 12.6 \\
\hline 900 & 22 & 308.2 & 322.9 & 271 & 14.3 & 14.0 & 12.6 \\
\hline 800 & 19 & 318.8 & 334.2 & 269 & 14.4 & 14.1 & 12.6 \\
\hline 700 & 17 & 331.3 & 347.4 & 267 & 14.5 & 14.2 & 12.6 \\
\hline
\end{tabular}

In the calculation of $\Delta_{3}$ it was assumed that $L=606.5$.

7 Robitzsch, op. cit. in fn. 2. 
It is easy to show that the variation of $\Delta T_{E}$ with pressure is slight and generallv of the same order of magnitude as the errors due to inaccuracies in the reading of the relative humidity and therefore in the calculated value of the water vapor content $w$. As an illustration some equivalent temperature differences have been calculated and reproduced in tables $1,2,3$. There is a slight increase of the equivalent temperature difference with decrease of pressure, but in all cases the variation caused by a drop in pressure from $1000 \mathrm{mb}$ to $700 \mathrm{mb}$ is less than four per cent of the total equivalent temperature difference.

The variation of the equivalent temperature difference with temperature can be seen from a comparison of table 1 with table 2, for corresponding pressures. Other quantities being equal, the equivalent temperature difference increases with increasing temperature, but the variation in $\Delta T_{E}$ corresponding to a temperature increase of $10^{\circ}$ is in all tabulated cases less than five per cent of the total value of $\Delta T_{E}$. Thus also the variation with temperature may generally be disregarded.

It follows from the preceding discussion that the variation of the equivalent temperature difference with temperature and pressure is such that this difference, ceteris paribus, is greater the lower the relative humidity. To understand this variation we must first consider the temperature at the condensation level. This temperature is determined by (19), which equation after substitution of $p_{d}$ and $T$ for $\theta_{d}$ may be written

$$
C \cdot \frac{e_{o m} k}{T_{o}}=\frac{w^{k} \cdot p d^{k}}{T},
$$

$C$ denoting a constant. The left side of this equation increases steadily with $T_{0}$. Thus, for a constant water vapor content $w$, the condensation temperature $T_{0}$ increases with increasing initial pressure $\left(p_{d}\right)$ of the element and decreases with increasing initial temperature $T$.

Now consider the following simplified process. A particle containing one gram of dry air and $w$ grams of water vapor is expanded adiabatically from $\left(p_{d}, T\right)$ to the condensation point $\left(p_{o d}, T_{0}\right)$. A definite, very small fraction of the moisture content, $\Delta w$, is then allowed to condense, precipitate and raise the temperature of the element by the amount $\Delta T_{o}=\frac{L_{0} \Delta w}{c_{p}}$. Thereupon the element is brought back, dry-adiabatically, to the original pressure $p_{d}$. To obtain the equivalent temperature difference $\Delta T_{E}$ for this condensation process it is necessary to calculate the amount of energy gained in the two adiabatic processes involved. This amount is obviously equal to

for the expansion and

$$
-c_{v}\left(T-T_{o}\right)
$$

$$
c_{\nu}\left[\left(T+\Delta T_{E}-\left(T_{o}+\Delta T_{0}\right)\right]\right.
$$

for the compression. From Poisson's equation it follows that these two quantities may be written

and

$$
-c_{v} T_{o}\left[\left(\frac{p_{d}}{p_{o d}}\right)^{k}-1\right]
$$

$$
c_{v}\left(T_{o}+\Delta T_{o}\right)\left[\left(\frac{p_{d}}{p_{o d}}\right)^{k}-1\right] .
$$


The algebraic sum of these two quantities,

$$
c_{v} \Delta T_{0}\left[\left(\frac{p_{d}}{p_{o d}}\right)^{k}-1\right]
$$

represents a gain and is stored up by the element as heat. By definition, the equivalent temperature difference is equal to the sum of the temperature increase due to condensation,

$$
\Delta T_{o}=\frac{L_{o} \Delta w}{c_{p}}
$$

and the temperature increase brought about mechanically,

$$
\Delta T_{o}\left[\left(\frac{p_{d}}{p_{o d}}\right)^{k}-1\right]
$$

Thus

$$
\Delta T_{E}=\Delta T_{o}\left(\frac{p_{d}}{p_{o d}}\right)^{k}=\frac{T}{T_{o}} \cdot \Delta T_{o}
$$

Now, if the initial pressure is kept constant while the initial temperature is raised, the temperature and therefore also the pressure at the condensation level decreases ( $w$ is all the time constant). Thus the net gain of work increases and the equivalent temperature difference increases. In the second place, assume the initial temperature $T$ to remain constant, while the initial pressure is decreased. The condensation temperature $T_{o}$ again decreases. It is easily seen that the work of compression as well as the work of expansion increases. The equivalent temperature difference increases as may be seen immediately

from the expression $\Delta T_{E}=\frac{T}{T_{o}} \cdot \Delta T_{o}$.

Thus the cause of the variation of the equivalent temperature difference with relative humidity lies in the fact that this difference, as here defined, measures not only the latent heat of the water vapor present but also a certain amount of work done by the surroundings on the element. This amount of work increases with decreasing relative humidity.

\section{Equivalent-Potential Temperature Diagrams}

A graphical representation of the equivalent-potential temperature is given in Plate I. The water vapor content is plotted along the horizontal axis. The partial potential temperature is set off, logarithmically, on the vertical axis. Since the latter quantity is connected with the specific entropy of dry air by the formula

$$
s=c_{p} \log \theta_{d}
$$

linear distances on the vertical axis represent differences in specific entropy.

The lines of constant equivalent-potential temperature ( $\theta_{E}$-lines) have been drawn with two degree intervals. The incomplete section at the bottom of the diagram represents saturated air at pressures higher than those normally occurring at sea level $(>1100 \mathrm{mb})$. 
In this diagram an unsaturated element of air undergoing adiabatic expansion will be represented by a characteristic point $\left(w, \theta_{d}\right)$, which will remain stationary until the level of condensation is reached. If the further expansion of the particle takes place pseudo-adiabatically, its characteristic point will move upward along the line of constant equivalent-potential temperature ( $\theta_{E}$-line) through the starting point. If the element is again compressed, its characteristic point will remain stationary at the last point reached during the preceding expansion, since no liquid water is available for evaporation and the compression therefore takes place dry-adiabatically.

\section{Characteristic Curves}

Temperature, pressure and humidity data obtained through aerological soundings in a given vertical air column may easily be converted into partial potential temperatures and water vapor contents by means of formulae (1) and (6). It should be noticed that this conversion can be made without a preceding evaluation of the altitudes corresponding to the various pressure levels. If the partial potential temperatures and water vapor contents for this particular sounding are plotted against each other on the equivalentpotential temperature diagram, one obtains the characteristic curve for this air column. Below some of the genetal properties of these curves are derived.

A thin horizontal stratum, within which the moisture content varies from $w$ to $w+d w$ and the potential temperature from $\theta_{d}$ to $\theta_{d}+d \theta_{d}$ may be represented on the equivalentpotential temperature diagram by a short straight line element. The layer may be compressed or expanded or it may be stretched or contracted laterally so that its vertical depth changes, but as long as these various changes take place adiabatically and no condensation occurs, the water vapor content and the partial potential temperature of each individual point will obviously remain unchanged. Thus the characteristic curve of the given infinitesimal layer will remain the same even though the latter may be stretched until its thickness becomes a fraction of what it originally was. This result holds also for finite vertical air columns consisting of a great number of superimposed very thin strata, each undergoing a different change of the type described above. Thus we find the following law:

I. The characteristic curve for a vertical air column of finite beight remains invariant with respect to arbitrary adiabatic deformations provided no condensation (or evaporation) takes place and provided no new strata are introduced or otbers made to disappear during the deformation of the original air column.

It is obvious that the invariance of the characteristic curve makes it an exceedingly useful tool in following the displacement of individual air masses across the synoptic chart. There are other elements which may be used for the same purpose (for instance, atmospheric suspensions of various kinds) but the two quantities selected above $\left(\theta_{d}\right.$ and $\left.w\right)$ are the only ones which result in a characteristic curve of definite dynamic significance. A similar method of identification, based on the construction of so-called ST-diagrams, has been in use in physical oceanography for years. Salinities and temperatures for corresponding depths are plotted against each other. In the diagram thus obtained, salinity obviously takes the place occupied by the moisture content in the atmospheric characteristic curve, since both indicate the percentual composition of the medium which is being studied. In the oceanographic problem there is no compressibility to consider; therefore the ordinary temperature may be used in place of the potential temperature. With the aid

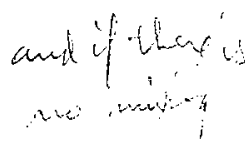


of ST-diagrams Helland-Hansen ${ }^{8}$ has been successful in establishing the Mediterranean origin of certain strata in the Atlantic, west of Gibraltar.

Next it may be asked how the characteristic curve varies from one part to another within a given air mass. As long as the air mass is strictly homogeneous, horizontally, the characteristic curve will obviously remain the same from one vertical air column to another. It was pointed out above that the characteristic curve for a given stratum is independent of its thickness. It follows that the characteristic curve will remain the same throughout the air mass even though the individual strata may vary in thickness from vertical to vertical (station to station), provided the succession of strata is exactly the same along each vertical. This property of the characteristic curves may also be stated in the following manner:

II. The characteristic curves for a given air mass will have overlapping parts which are identical provided the surfaces of constant partial potential temperature and the surfaces of constant water vapor content coincide throughout the air mass.

\section{Stratificatron}

It is well known that if a given air layer of finite thickness is thoroughly stirred and if the amount of moisture present is so small that no condensation takes place, then the layer will gradually assume an adiabatic temperature lapse rate while its water vapor content per unit mass $(w)$ approaches a constant value. This development results from the effect of turbulence in carrying water vapor and potential temperature in the direction of the gradient of these elements. The above result may be expressed in the following law.

III. The characteristic curve for a tborougbly stirred unsaturated layer limited above and below by planes through which no transport of heat or water vapor takes place, reduces to a point.

A stable air layer characterized by a number of inversions and a rapid decrease of water vapor content upward, obviously has an elongated characteristic curve. To obtain a picture of the stratification of a given air column from its characteristic curve one may interpolate, from the original data, the pressures, temperatures, and relative humidities for $0.5,1,2,3 \ldots \mathrm{km}$. elevation above sea level and convert these values into water vapor contents $(w)$ and partial potential temperatures $\left(\theta_{d}\right)$. It is then possible to mark, on the characteristic curve, the points corresponding to the above standard elevations. These points shall be referred to below as standard characteristic points. The preceding discussion may be summarized in the following law:

IV. The more crowded the standard characteristic points, the more homogeneous (stirred) the air mass; the further apart these points are, the more pronounced is the stratification.

It is necessary to consider the effect of stirring also in those cases when condensation occurs. If the layer under consideration is limited above and below by planes through which no transport of water vapor or realized heat takes place, stirring will first bring about condensation in a narrow layer. next to the top. Within this layer a water vapor gradient upward $\left(\frac{d w}{d z}<0\right)$ is established which, because of the turbulent stirring, gradually spreads downward into the unsaturated strata. Thus an upward flow of water vapor and therefore also of latent heat is established.

${ }^{8}$ Helland-Hansen, B. and Nansen, F., The Eastern North Atlantic, Geofysiske Publikasjoner, Vol. IV, No. 2, OsIo, 1925. For additional references see Defant, A., Dynamische Ozeanographie, p. 95, Einfiibrung in die Geopbysik, III, Springer, Berlin, 1929. 
The condensation and precipitation of liquid water at the upper boundary is attended by a liberation of latent heat and therefore also by an increase of the partial potential temperature. Thus a downward gradient of the partial potential temperature and consequently a downward flow of realized heat is established. In the particular case under discussion complete equilibrium is not reached until so much liquid water has left the system that the water vapor barely reaches saturation at the top and $w$ and $\theta_{d}$ have constant values throughout the whole column.

However, long before this equilibrium is reached a practically steady state develops in which the upward flow of latent heat is approximately equal to and balanced by the turbulent downward transport of realized heat. Per unit area and time the upward flow of latent heat has the value $-c L \frac{d q}{d z}, c$ representing the "eddy conductivity" for water vapor of the air layer under discussion and $q$ as before the specific humidity. This value is obtained by multiplying the well-known expression for the transport of water vapor ${ }^{9}$ in the latent heat of water vapor (considered constant). The transport of latent heat is, under the conditions here assumed, constant from the lower boundary of the layer under consideration and up to the condensation level.

The turbulent flow of realized heat is of a more complicated nature. On account of the release of latent heat above, the air column must have a stable stratification. The constant stirring of the layer then requires a steady supply of work which is continually being transformed into heat. This heat is added to the downward flow of heat emanating from the condensation level. Therefore, the heat transport varies somewhat from level to level and has its maximum below. A detailed analysis of this question is beyond the scope of the present paper; it has been treated by L. F. Richardson. ${ }^{10}$ Equalizing the downward flow of realized heat at the condensation level with the upward flow of latent heat and using Richardson's expression for the eddy-flux of heat we find, just below the condensation level,

$$
c \cdot c_{p} \frac{T}{\theta} \frac{d \theta}{d z}=-L \cdot c \cdot \frac{d q}{d z}
$$

In this equation the two c's introduced represent the eddy conductivities for heat and water vapor respectively. It is generally assumed that these coefficients are identical; they may then be dropped from the equation just given. If $T$ is regarded as constant $\left(T=T_{0}\right)$ and if furthermore, the small differences between $\theta$ and $\theta_{d}$ and between $w$ and $q$ are disregarded we find, in the vicinity of the condensation level and, with a fair degree of accuracy, throughout the whole air column below,

$$
\frac{d \log \theta_{d}}{d z}+\frac{L}{c_{p} T_{o}} \frac{d w}{d z}=0
$$

or

$$
\theta_{d} \mathrm{e}^{\frac{L w}{c_{p} T_{o}}}=\text { constant }=\theta_{E}
$$

\footnotetext{
${ }^{9}$ Richardson, L. F., Weather Prediction by Numerical Process, pp. 65-71, Cambridge University Press, 1922.

${ }^{10}$ Richardson, op. cit. in fn. 9, pp. 70-71.
} 
V. The equivalent-potential temperature remains approximately constant also witbin the lower unsaturated layer, the water vapor content decreasing upward and the partial potential temperature increasing upward in such a fashion that the upward flow of latent beat and the downward flow of realized beat balance each other.

Verifications of this law will be found in chapter III.

We have thus found, on theoretical grounds, that thoroughly stirred strata, regardless of their degree of saturation, necessarily are characterized by a fairly constant equivalent potential temperature, i.e., their characteristic curves must follow the $\theta_{E}$-lines in the equivalent-potential temperature diagram.

In case of a saturated air layer in pseudoadiabatic equilibrium it is easily shown that the standard characteristic points are distributed in a definite fashion along the characteristic curves (here coinciding with $\theta_{E}$-lines). That such must be the case follows from the fact that during a pseudoadiabatic expansion there is a definite relation between pressure, (partial) potential temperature and water vapor content. Combining this relation with the hydrostatic equation we may eliminate the pressure and insert instead the altitude.

The relation between pressure and partial potential temperature referred to above makes it possible for us to enter the isobaric lines corresponding to pseudoadiabatic equilibrium on the equivalent-potential temperature diagram. This has been done on the diagram reproduced in Plate I. At first the isotherms were calculated with the aid of (19). For each isotherm the value of the left member of this equation was determined. Then $\theta_{d}$ was determined for different values of the moisture content $(W)$. Having entered the isotherms on the equivalent-potential temperature diagram, the isobars are easily plotted by means of Poisson's equation,

$$
\theta_{d}=T \cdot\left(\frac{P}{p_{d}}\right)^{k}
$$

the factor $\left(\frac{P}{p_{d}}\right)^{k}$ being constant along any isobar. As a check, certain isobars were re-calculated from

$$
W=\frac{622}{p_{d}} e_{m}
$$

$e_{m}$ being a function of the temperature alone. Both isobars and isotherms are printed in red on the diagram.

To obtain the distribution of the standard characteristic points it is convenient to start from the expression for the variation in geopotential on the temperature-entropy diagram ${ }^{11}$, namely

$$
g d z=c_{p} T \frac{d \theta}{\theta}-c_{p} d T
$$

in which expression the partial potential temperature may be introduced from

$$
\theta_{d}=\theta\left(1+\frac{m_{d}}{m_{w}} w\right)^{k}
$$

11 Shaw, Sir Napier, Manual of Meteorology, Volume III, The Physical Processes of Weather, p. 298, Cambridge
ersity Press, 1930. University Press, 1930. 
Thus

$$
g d z=c_{p} T \frac{d \theta_{d}}{\theta_{d}}-c_{p} d T-T \frac{R}{m_{w}} \frac{d w}{1+\frac{m_{d}}{m_{w}} w},
$$

the third term in the right number usually being small in comparison with the two first terms. To eliminate $d T$ we may use the differential form of the equation for pseudoadiabatic equilibrium

$$
\frac{d \theta_{d}}{\theta_{d}}+d\left(\frac{L w}{c_{p} T}\right)=0
$$

Here $T$ has been introduced instead of $T_{o}$ since we are dealing with a saturated atmosphere. Assuming $L$ and $c_{p}$ to be constant, this gives

$$
\frac{d T}{T}=\frac{d w}{w}+\frac{c_{p} T}{L w} \frac{d \theta_{d}}{\theta_{d}}
$$

Therefore,

$$
g d z=c_{p} \cdot T\left[\frac{d \theta_{d}}{\theta_{d}}-\frac{c_{p} T}{L w} \frac{d \theta_{d}}{\theta_{d}}-\frac{d w}{w}-\frac{R}{m_{w} c_{p}} \frac{d w}{1+\frac{m_{d}}{m_{w}} w}\right]
$$

or, after some substitutions,

$$
g d z=c_{p} T\left[\frac{d \theta_{d}}{\theta_{d}} \frac{\log \theta_{E}-\log \theta_{d}-1}{\log \theta_{E}-\log \theta_{d}}-\frac{d w}{w} \frac{1+\frac{m_{d}}{m_{w}}(1+k) w}{1+\frac{m_{d}}{m_{w}} w}\right]
$$

The air being saturated, one may eliminate $T$ from the above formula by means of (27).

The above expression for the geopotential may be simplified if it is remembered that, very nearly,

$$
\frac{1+\frac{m_{d}}{m_{w}}(1+k) w}{1+\frac{m_{d}}{m_{w}} w}=1+\frac{R}{m_{w} c_{p}} w \approx 1
$$

Thus, approximately,

$$
g d z=c_{p} T\left[\frac{d \theta_{d}}{\theta_{d}} \frac{\log \theta_{E}-\log \theta_{d}-1}{\log \theta_{E}-\log \theta_{d}}-\frac{d w}{w}\right] .
$$

VI. If an air column is in pseudoadiabatic equilibrium its standard characteristic points are separated by definite intervals which may be once for all entered upon the equivalent-potential temperature diagram.

\section{StABILITY}

It is customary to indicate the stability of a given air layer by the behaviour of a small atmospheric element which has been lifted, adiabatically or pseudoadiabatically, from its equilibrium position, while the atmosphere as a whole remains at rest. In this way one is led to distinguish between three different degrees of stability. 
(1) Absolute Instability. The temperature lapse rate is greater than the dry-adiabatic $\left(\frac{g}{c_{p}}\right)$. In this case, regardless of the moisture content of the air but assuming that viscous forces may be neglected, any small impulse is sufficient to set a particle of air into motion at an increasing speed away from its original equilibrium position.

(2) Conditional Instability. The temperature lapse rate is less than the dry-adiabatic and higher than the condensation- or pseudoadiabatic lapse rate. In this case the atmosphere is stable with respect to impulses tending to displace air particles downward. If the relative humidity is high a sufficiently strong impulse upward may set an air particle in flight away from its original position. The magnitude of the impulse required decreases with increasing relative humidity and varies also with the values of the actual and condensation-adiabatic lapse rates at the point under consideration. Conditional instability is of no significance in case of very dry air since then the impulses required to upset the equilibrium are very great.

(3) Absolute Stability. The temperature lapse rate is less than the condensation adiabatic (pseudoadiabatic).

In the second and third cases the potential temperature increases with elevation, in the first case it decreases upward. It is, of course, possible to tell from the characteristic curve if the potential temperature (strictly speaking the partial potential temperature) decreases or increases with elevation, but it is not always possible to say whether the atmosphere is conditionally unstable or absolutely stable.

It is, however, feasible to discuss from another point of view the stability of a given air column by means of its characteristic curve. Assuming a normal vertical distribution of the water vapor content, that is, a decrease of $w$ with altitude, we may distinguish between the three following cases.

1. The characteristic curve slopes down towards the lower left corner of the equivalent-potential temperature diagram. In this case the partial potential temperature decreases with elevation. This case represents absolute instability as described above.

2. The characteristic curve ascends to the left but its slope is less than that of the pseudoadiabatic lines; thus the equivalent potential temperature $\left(\theta_{E}\right)$ decreases with elevation. In this case a layer of finite depth, lifted by convection adiabatically, and eventually pseudoadiabatically towards higher levels, must gradually become absolutely unstable. The process is illustrated in figure 1 . We may term this case convective instability. It is possible to estimate, from the equivalent-potential temperature diagram, the decrease in pressure required to bring about absolute instability. Convective instability may or may not be accompanied by conditional instability.

Figure 1 is part of an equivalent-potential temperature diagram on which has been plotted part of the characteristic curve for Broken Arrow on December 13, 1929. The layer to be analyzed extends from the $925 \mathrm{mb}$. to the $797 \mathrm{mb}$. isobaric surface (these values refer to the partial pressures of the dry air). The atmosphere is saturated from the base up to the $880 \mathrm{mb}$. level. At the top (797 mb.) the relative humidity is about 72 per cent. The whole layer is characterized by convective instability since the equivalent-potential temperature drops from about 334.5 at the lower boundary to about 227.5 at the upper boundary.

The top point of the layer falls on the $740 \mathrm{mb}$. isobar in the diagram. This indicates that the partial pressure of the dry air must be reduced adiabatically to $740 \mathrm{mb}$. in order to bring about condensation at the upper boundary of the layer under consideration. 
This may be accomplished by a bodily lifting of the whole layer. Such lifting would reduce the pressures at the lower boundary and at the intermediate point by approximately the same amount as at the top, namely $57 \mathrm{mb}$. (The last statement is strictly true only with reference to the total pressure-dry air pressure plus partial pressure of water vaporand then only when the removal of mass by precipitation may be neglected.) Since the lower part of the layer is saturated, the lifting results in a displacement of the corresponding characteristic points along the pseudoadiabatic lines to the proper pressures, $823 \mathrm{mb}$. and $868 \mathrm{mb}$. The characteristic point for the top surface remains stationary.

In its new position the layer is saturated throughout. It is easy to see that the difference in partial potential temperature between the two boundaries has decreased.

Additional bodily and pseudoadiabatic lifting will displace the characteristic curve of the layer in the manner indicated by the various positions on the diagram. Finally, when the top surface reaches the $300 \mathrm{mb}$. level, the partial potential temperature of the

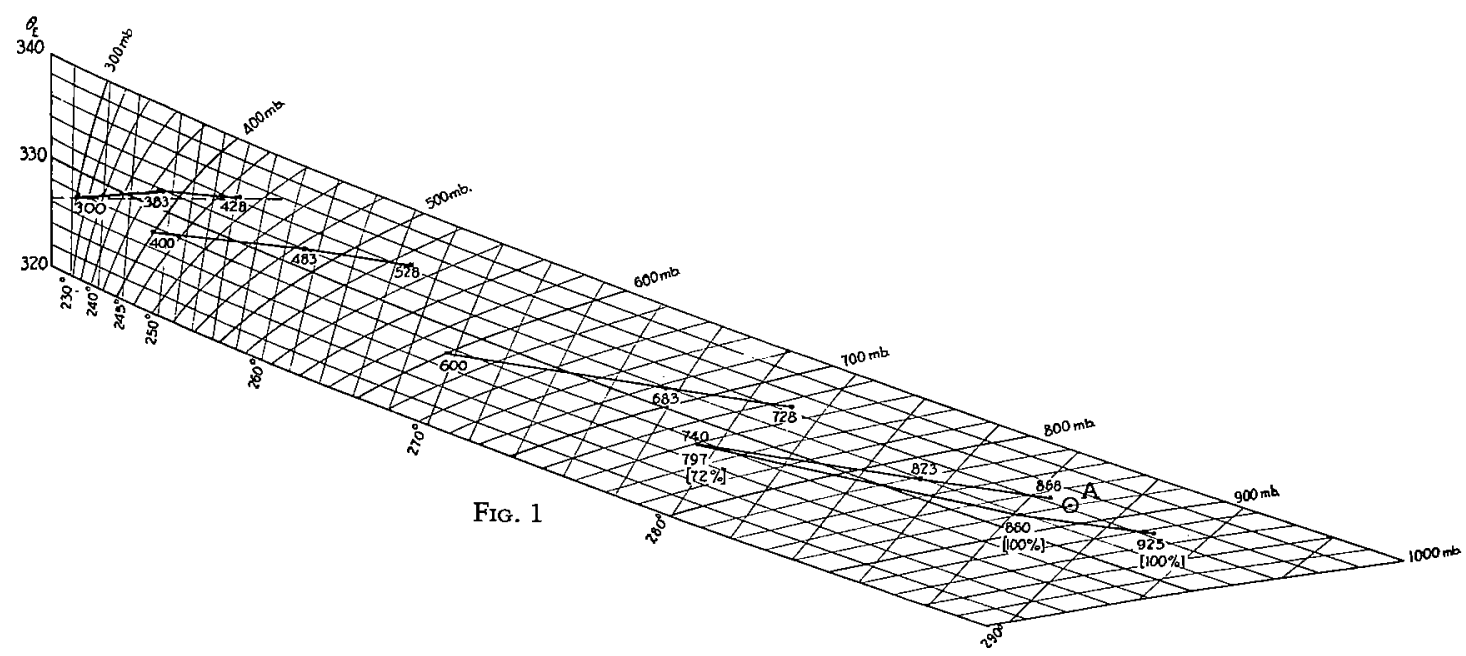

upper boundary is lower than that of the intermediate point and about the same as that of the lower boundary, that is, even if its saturated state be distegarded, the layer is now absolutely unstable. The broken line on the diagram represents a constant partial potential temperature line.

A saturated layer is absolutely unstable whenever the equivalent-potential temperature decreases with elevation. This is easily shown graphically. At first it may be pointed out that the actual pressure in any point on a characteristic curve always must be equal to or higher than the value of pressure on the printed isobar going through the point in question, since the latter indicates the pressure at the level to which the point must be lifted in order to reach condensation. In the case of saturation, actual pressures and those indicated on the equivalent-potential temperature diagram are identical.

If we now return to the lower part of the layer analyzed in figure 1 , it is easily seen that a small particle of air from the lower boundary ( $925 \mathrm{mb}$.), if displaced pseudoadiabatically to the $880 \mathrm{mb}$. level (point $\mathrm{A}$ ), will have a higher partial potential temperature than the one prevailing in the surrounding atmosphere at this level. This is evident from the fact that the characteristic point of the surroundings (marked 880 ) and 
that of the moving particle (A) are located on the same isobar, which ascends in a uniform fashion from left to right.

The characteristic curve in figure 2 was obtained from part of the ascent at San Diego on April 3, 1930. Also here the atmosphere is characterized by convective instability. However, in this case the decrease in equivalent-potential temperature with elevation is due to an extremely rapid decrease in $w$, which is not quite offset by a rapid increase in the potential temperature. The atmosphere is dry and warm at higher levels and therefore absolutely stable. This is easily verified by the diagram.

If an element from the bottom layer ( $897 \mathrm{mb}$.) is lifted adiabatically it will not reach saturation until the pressure drops to about $851 \mathrm{mb}$. Thus, at the $867 \mathrm{mb}$. level its characteristic point still remains in its original position, while the characteristic point for the surroundings shows a decidedly higher potential temperature. If the same element is lifted all the way to the top surface $(780 \mathrm{mb}$.) its characteristic point is displaced to $A$, where the potential temperature still is far below that of the surroundings. It is also easy to establish the fact that one would have to lift the layer a considerable distance in order to "realize" its convective instability.

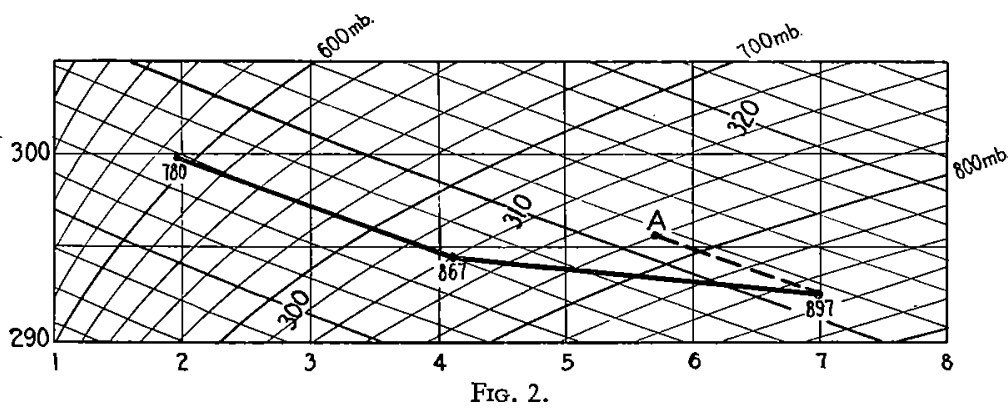

3. The characteristic curve ascends to the left and its slope is steeper than that of the $\theta_{E}$-lines, so that the equivalent potential temperature increases with elevation. In this case, a horizontal layer of finite thickness which is lifted at first adiabatically and beyond the condensation point pseudoadiabatically to higher levels must eventually become absolutely stable. We may term this case convective stability.

It is easy to prove that a layer must be absolutely stable - even without lifting whenever the equivalent-potential temperature increases with elevation in a monotone fashion, i.e. it may be shown that convective stability carries with it absolute stability. The proof may be found in figure 3. The line $A B C$ represents the observed characteristic curve, the equivalent-potential temperature increasing upward. We make the assumption that each point on the characteristic curve corresponds to one fixed level in the atmosphere; thus we exclude those cases in which the atmosphere contains finite strata of constant water vapor content and constant partial potential temperature. The actual pressure in point $A$ must be equal to or more than $800 \mathrm{mb}$, the latter value representing the pressure on the printed isobar through $A$. If a unit particle of air at the level corresponding to point $A$ is lifted adiabatically its characteristic point will at first remain in $A$. The characteristic point of the surrounding atmosphere is displaced along the curve $A B C$, that is, towards higher partial potential temperatures. Thus, for displacements not reaching the condensation level, the moving element will always have a lower partial potential temperature and therefore a lower actual temperature than the surroundings 
and the element will return to its original position. If the particle is lifted beyond the condensation level, the continued displacement takes place pseudoadiabatically and the characteristic point of the particle moves along the $\theta_{E}$-line through $A$. If the particle is lifted to the $700 \mathrm{mb}$. level, its characteristic point will be displaced to $D$. The printed isobar through $D$ intersects the characteristic curve in $B$. In point $B$, the actual pressure must be equal to or higher than $700 \mathrm{mb}$. Thus, the characteristic point of the $700 \mathrm{mb}$. level in the resting atmosphere must be located in $B$ or somewhere above $B$ on the observed characteristic curve, say in $D^{\prime}$. But the partial potential temperature, and therefore the actual temperature, is obviously lower in $D$ than in $B$ and a fortiori lower than in $D^{\prime}$. Thus the displaced element will have a tendency to drop back to its original level, that

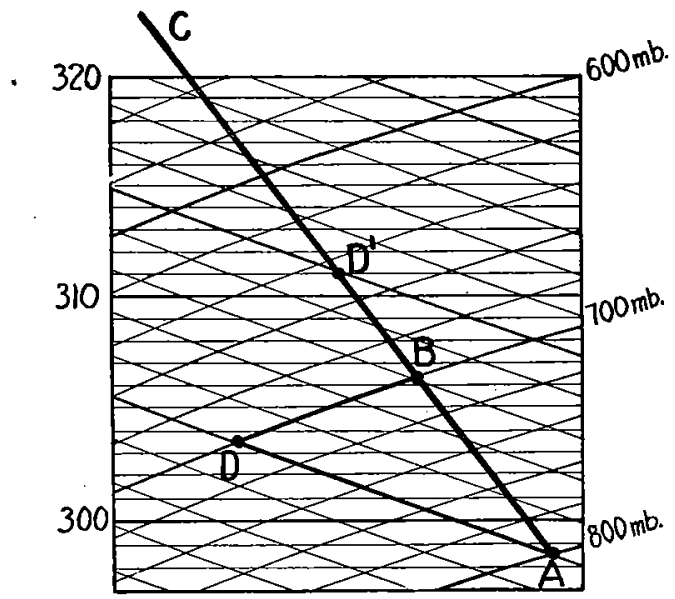

Fig. 3

is, the atmosphere is absolutely stable. This absolute stability within the layer under consideration cannot be destroyed by adiabatic or pseudoadiabatic lifting or lowering or by cross-section changes of the air column.

VII. The characteristic curve permits us to recognize absolute instability $\left(\frac{d \theta}{d z}<0\right)$, convective instability $\left(\frac{d \theta_{E}}{d z}<0\right)$, and convective stability $\left(\frac{d \theta \mathrm{E}}{d z}>0\right)$. Convective stability carries with it absolute stability, regardless of the state of saturation of the air column, and this absolute stability cannot be destroyed by any orderly isentropic process performed with the air. If a layer in a state of convective instability is lifted solidly to bigber levels it will eventually become absolutely unstable.

\section{Fronts and Inversions in the Characteristic Curves}

The moisture in the atmosphere has its origin at the earth's surface. Within an air mass moisture is carried to higher levels by turbulence (convection). This upward flow of moisture continues only as long as the water vapor gradient is directed upward. It is therefore obvious that turbulent transport alone never can produce a maximum of the water vapor content somewhere in the free atmosphere. 
The removal of moisture from the atmosphere through precipitation usually takes place at fairly high levels. No rapid removal on a large scale occurs in strata adjacent to the ground. Thus we may expect that within a given air mass the water vapor content decreases gradually with elevation, that is, the characteristic curve should run from right to left on the equivalent-potential temperature diagram.

In a front, the moist surface air of a warmer current rests on a wedge of somewhat colder and drier air. A characteristic curve obtained from a sounding through a front may therefore be expected to exhibit a well-marked maximum somewhere in midair. At a warm front the moist air ascends from the surface layer of the warm sector over a wedge of colder air. Since this ascent takes place in an approximately pseudoadiabatic fashion, it means that the equivalent potential temperature in the front itself must have the value characteristic of the surface air in the warm sector, provided the ascending air really originates at the surface.

Subsidence inversions, on the other hand, are the result of the sinking and spreading out of air from higher levels over cushions of stagnant air next to the ground. They are accompanied by marked drops in relative and also specific humidity. An air mass containing subsidence inversions will therefore have a normal characteristic curve with a more or less regular decrease of the water vapor content.

VIII. Characteristic curves enable us to distinguish between fronts and inversions. A characteristic curve obtained from a sounding through a front is wedge-shaped and shows a well-marked vapor content maximum somewhere in midair. In a warm front the equivalent-potential temperature at the point of maximum water vapor content has the value characteristic of the surface air in the following warm sector. Characteristic curves from air masses containing subsidence inversions displav no maximum but run in a regular fashion from right to left on the equivalent-potential temperature diagrams.

Verifications of this law may be found in Chapter III. 


\section{Chapter II}

\section{THE EQUIVALENT-POTENTIAL TEMPERATURE AND THE TOTAL ENERGY RELEASED BY CONVECTION}

In recent years two methods have been developed to make possible the forecasting of convection from estimates of the potential energy and heat, realized or latent, available in the atmosphere. These estimates are obtained by a study of temperature-entropy or temperature-log pressure diagrams. ${ }^{12}$ In both cases one determines graphically the amount of energy set free through the isentropic vertical displacement of an arbitrarily selected unit atmospheric element, while the atmosphere as a whole is assumed to be at rest. This energy is represented by an area on the diagram. It is important to bear in mind the restriction to unit elements, since, in consequence of this restriction, the results obtained necessarily must depend upon the original position and properties of the chosen air particle. This is particularly noticeable in those cases when the surface air layer has absorbed large quantities of heat and moisture from the ground while the free atmosphere is dry and fairly stable. Under such circumstances either diagram would indicate a large amount of available energy ("positive area") for surface points, but any other element, representing air forced to ascend against stabilizing forces, would show a "negative area.", Thus, in order to form a complete picture of the energy released through convection it is necessary to study the displacement of a number of elements originating at various levels throughout the ait column.

Another situation in which the consideration of a single point might tend to produce misleading results, occurs when a cold and fairly dry surface layer is overrun by warm, moist air characterized by conditional instability. During the winter season it often happens that a shallow layer of transitional polar air of Canadian or maritime origin stagnates over the Southeastern states, while a strong southwest wind at the one or two kilometer level indicates overrunning by Gulf air. In this case a study restricted to the displacement of surface elements alone would fail to disclose the pronounced instability of the upper air; although this instability often leads to the development of vigorous warm-front thunder storms.

A fair estimate of the thickness and relative importance of the various strata which contribute to the maintenance of convection is of particular importance whenever one considers conditions over a large area since it then may be assumed that the convection currents within the region in question necessarily must be fed by the available potential and internal energy within the same space and by the latent heat of the water vapor therein.

This does not apply in case of convergent airflow and fairly stationary conditions, since convective kinetic energy then may be supplied by air drawn in from the sides. Then the indication of available energy (positive area) by even a single reference point may be important enough to warrant consideration.

Even if judicious use is made of the thermodynamic methods referred to above, the problem of determining the chances of convection is by no means solved. Although the entropy diagram may fail to indicate the existence of available energy at any level, it is still possible that a portion of the atmosphere may be convectively unstable (see page 20).

12 Shaw, op. cit. in fn. 11, Chapter VII; also Refsdal, A., Der Feuchtlabile NiederschIag, Geofysiske Publikasjoner, Vol. V, No. 12, Oslo, 1930. 
that is, this portion of the atmosphere, if lifted solidly, will gradually acquire a lapse rate steeper than the condensation-adiabatic, and eventually steeper than the dry-adiabatic so that finally spontaneous convection will set in within the layer. Such a possibility must be considered particularly in connection with warm fronts, since the overrunning Gulf air generally is characterized by convective instability. This steepening of the lapse rate within the lifted layer is accentuated if the flow takes place in such a fashion that the horizontal cross section. of the moving layer decreases, that is, if the stream lines in a vertical plane converge upward. Obviously the nature of convection is not such that a large body of air is lifted solidly to higher levels, but it is equally plain that we can not hope to find a dependable criterion for convection on the highly artificial assumption that one small element at a time is lifted while the rest of the atmosphere remains in equilibrium.

It is evident that a method is needed which would make it possible to calculate the total amount of energy available for convection or, if this should not be feasible, would enable us to estimate the chances of convection from a consideration of the stability of the air column as a whole rather than of arbitrarily chosen elements thereof.

In this connection the classical investigation by Margules ${ }^{13}$ of the total available kinetic energy of certain atmospheric systems naturally suggests itself as a pattern along which a method of attack might be developed. Margules, it may be recalled, computes the total potential and internal energy in a system in which colder air rests side by side with, or above, warmer air, the two masses being separated by a thin wall. After removal of the wall, the system assumes a stable equilibrium position with the air arranged in horizontal strata so that the potential temperature increases upward. The difference between the total energy in the original and in the final stage represents the available energy.

For the moment we may restrict ourselves to the case of a dry atmosphere. In order to apply Margules' method to the problem of local heat convection, we would then have to calculate the total (potential plus internal) energy of an air column of arbitrary temperature distribution and then repeat the calculation after a rearrangement without mixing of the individual infinitesimal strata according to their potential temperature. The difference between the two results would represent the total convective energy.

The basic assumption upon which this method rests; namely, that the individual, infinitely thin strata rearrange themselves witbout mixing, may be acceptable in case of atmospheric systems of the order of magnitude of the average extratropical cyclone, but we know from experience that local convection eventually results in a fairly thorough mixing of a portion of the lower atmosphere. To make the discussion more definite, let us consider a dry air column in unstable (superadiabatic) equilibrium between the ground and the elevation $h$; above this height the lapse rate is stable. A slight disturbing force will then bring about overturning of the lower unstable layer. A tendency towards a rearrangement of the individual strata undoubtedly exists but the potential (and internal) energy set free during this rearrangement and fitst appearing as convective (turbulent kinetic) energy is used to thoroughly mix the various strata participating in the overturning, stirring also part of the upper, stable atmosphere. This stirring and mixing will go on until all the convective energy is consumed.

Thus an air column having a superadiabatic lapse rate between the ground and the elevation $h$, if permitted to overturn, will rearrange itself, not according to the potential

${ }^{13}$ Margules, M., Über die Energie der Stürme, Jabrbucb d.k.k. Zentralanst. f. Met. und Geodyn., Wien, 1903. 
temperature of the individual strata but in such a fashion that a constant potential temperature is established from the ground and up to an elevation $H(>b)$; above this level no change will take place with the exception of a slight vertical displacement of the upper atmosphere as a whole. We may assume, as a first approximation, that all the energy originally set free in the final stage has been consumed in the building up of an adiabatic lapse rate through the upper, stable strata. Hence, the total (potential and internal) energy must be the same in the final as in the original stage.

The total energy in the final stage will depend upon the depth $(H)$ and the potential temperature $(\theta)$ of the convective layer. There are infinitely many pairs of values of $\theta$ and $H$ which satisfy the energy requirement stated above. Energy considerations alone will therefore not enable us to determine the value of $H$. However, from these infinitely many pairs of $\theta$ and $H$-values it is possible to single out one pair by impressing the additional requirement that in its final stage the system must have a maximum of entropy. This condition may at first appear contradictory to the preceding discussion, since the convective layer then necessarily would be isothermal. We must therefore supplement our condition by the additional assumption that a minute fraction of the energy originally liberated still remains in existence as convective (turbulent kinetic) energy, keeping the air in adiabatic equilibrium (thoroughly stirred) against the temperature-equalizing influence of molecular heat conduction. Considering the slowness of the latter process in the atmosphere the amount of convective energy required for this purpose is obviously very slight.

The problem thus consists in determining the final depth $H_{f}$ and the final potential temperature $\theta_{f}$ of a dry air layer in convective equilibrium, having the same total energy as before its overturning and having an entropy maximum compatible with adiabatic equilibrium.

Before we attempt to include in our discussion the effect of water vapor, we shall formulate the above problem analytically and show how it may be solved.

In the initial stage the potential energy of an air column extending from the ground up to an arbitrary elevation $b$ is,

$$
P E=\int_{0}^{b} g z \rho d z=-b \cdot p h+\int_{0}^{b} p d z .
$$

The internal energy is

$$
I E=\int_{0}^{b} c_{v} T \rho d z=\frac{m c_{v}}{R} \int_{0}^{b} p d z
$$

and thus the total energy

$$
E^{\prime}=-b \cdot p_{b}+\frac{m c_{p}}{R} \int_{0}^{b} p d z=-b \cdot p_{b}+\frac{c_{p}}{g} \int_{p_{b}}^{p_{0}} T d p .
$$

We may now choose for $p_{b}$ the value of the pressure at the level to which the final convective layer will reach. Beyond this elevation, the internal structure of the atmosphere will remain undisturbed by the overturning. In our energy calculations it is therefore permissible to substitute, for this upper atmosphere, a weight $p_{b}$. Since the upper atmosphere may be displaced upward or downward as a solid during the convective 
process, it is necessary to include in the total energy of the system the potential energy of this weight, namely $b \cdot p_{b}$. By adding this term to $E$ we find the total energy of our system to be

$$
E=\frac{c_{p}}{g} \int_{p b}^{p_{0}} T d p
$$

and this quantity must remain the same before and after the convective process.

To simplify. the following calculations it is advisable to introduce a new variable

$$
x=\frac{p}{p_{0}} .
$$

Then

$$
\frac{E}{\frac{c p p_{0}}{g}}=U=\int_{x b}^{1} T d x
$$

Introducing the potential temperature and choosing $P(=1000 \mathrm{mb}$.) as standard pressure we find

$$
U=l \int_{x_{b}}^{1} \theta x^{k} d x
$$

The specific entropy $s$ of dry air is calculated from

$$
s=c_{p} \log T-\frac{R}{m} \log \frac{p}{P}=c_{p} \log \theta
$$

when referred to $T=1$ and $p=P$. The total entropy is

$$
S=\int_{0}^{b} s \rho d z=\frac{1}{g} \int_{p_{b}}^{p_{o}} s d p=\frac{c_{p} \cdot p_{o}}{g} \int_{x b}^{1} \log \theta \cdot d x .
$$

Thus

$$
\frac{S}{\frac{c_{p} p_{0}}{g}}=Z=\int_{x b}^{1} \log \theta \cdot d x .
$$

In the final stage the potential temperature is constant with elevation and equal to $\theta_{f}$. By inserting this constant value in (7) we find that the final energy is proportional to

Since

$$
U_{f}=l \theta_{f} \cdot \frac{1}{k+1}\left[1-x_{b}^{k+1}\right]
$$

$$
U_{f}=U
$$

it follows, from (7), that

$$
\theta_{f}=\frac{k+1}{1-x_{b}^{k+1}} \int_{\left(x_{b}\right)}^{(1)} \theta d\left(\frac{x^{k+1}}{k+1}\right)
$$

From (10) it follows that the final value of the entropy is proportional to

$$
Z_{f}=\left(1-x_{b}\right) \log \theta_{f}
$$


By substitution we find

$$
Z_{f}=\left(1-x_{b}\right) \log \left[\frac{k+1}{1-x_{b}^{k+1}} \int_{\left(x_{b}\right)}^{(1)} \theta d\left(\frac{x^{k+1}}{k+1}\right)\right] .
$$

We must now introduce our second requirement. If the entropy of the undisturbed air above the convective layer be indicated by $S_{a}$, we have before the overturning

$$
S^{\text {total }}=S+S_{a}
$$

after the overturning

$$
S_{f}^{\text {total }}=S_{f}+S_{a}=S_{f}+S^{\text {total }}-S .
$$

Now, $S_{f}^{\text {total }}$ must have a maximum value. Since $S^{\text {total }}$ is constant, we maysubstitute the condition that $S_{f}-S$ or $Z_{f}-Z$ must have a maximum value.

But $Z_{f}-Z$ has the value

$$
\Delta=Z_{f}-Z=\left(1-x_{b}\right) \log \left[\frac{k+1}{1-x_{b}^{k+1}} \int_{\left(x_{b}\right)}^{(1)} \theta d\left(\frac{x^{k+1}}{k+1}\right)\right]-\int_{x_{b}}^{1} \log \theta \cdot d x .
$$

In this expression $\theta$ represents the original potential temperature and $\Delta$ is therefore a function of $x_{b}$ alone. Thus, if $\Delta$ is to have a maximum value, the two conditions

$$
\left(\frac{d \Delta}{d x}\right)_{x=x_{b}}=0, \quad\left(\frac{d^{2} \Delta}{d x^{2}}\right)_{x=x_{b}}<0
$$

must be fulfilled.

Introducing a new variable

$$
\beta=\frac{1-x^{k+1}}{k+1},
$$

we may write

$$
\Delta=\left(1-x_{b}\right) \log \frac{1}{\beta_{b}} \int_{0}^{\beta_{b}} \theta d \beta-\int_{x_{b}}^{1} \log \theta d x .
$$

Differentiation gives

$$
\frac{d \Delta}{d x_{b}}=-\log \frac{\int_{0}^{\beta_{b}} \theta d \beta}{\beta_{b} \theta_{b}}-\frac{(k+1) x_{b}{ }^{k}\left(1-x_{b}\right)}{1-x_{b}^{k+1}}\left[\frac{\beta_{b} \theta_{b}}{\int_{0}^{\beta_{b}} \theta d \beta}-1\right]
$$

$$
\frac{d \Delta}{d x_{b}}=\log M-r(M-1)
$$

$r$ and $M$ being abbreviations for

$$
\begin{aligned}
r & =\frac{(k+1) x_{b}^{k}\left(1-x_{b}\right)}{1-x_{b}^{k+1}}, \\
M & =\frac{\beta_{b} \theta_{b}}{\int_{0}^{\beta_{b}} \theta d \beta} .
\end{aligned}
$$


It is easily seen that $\frac{d \Delta}{d x_{b}}$ is equal to zero if

$$
M=\frac{\beta_{b} \theta_{b}}{\int_{0}^{\beta_{b}} \theta d \beta}=1 .
$$

It may be shown that the particular solution just given then and then only represents an entropy maximum when the condition

$$
\left(\frac{d \theta}{d x}\right)_{x=x_{b}}<0
$$

is fulfilled. ${ }^{14}$ From this inequality may be derived another,

$$
\left(\frac{d \theta}{d z}\right)_{z=b}>0
$$

14 Equation (19) may possibly have other roots than $M=1$. By introducing the symbols $M$ and $r$ we may give this equation the form

(19b) $\quad \log M-r(M-1)=0$,

or, after division by $M-1$,

$$
\text { (19c) } \frac{\log M}{M-1}=r \text {. }
$$

It is easy to see that $r$ is a positive quantity, increasing in a monotone fashion from $r=0$ for $x=0$ to $r=1$ for $x=1$. Thus $r$ is always less than 1 (except at the ground, $x=1$ ). Also $M$ is, by definition, positive. When $M$ is smaller than $1, \frac{\log M}{M-1}$ is greater than 1 , demonstrating that there are no roots $M<1$. The left member of (19c) becomes smaller than 1 for $M-$ values in excess of 1 . Thus there may possibly exist roots $M>1$. From a comparison of (13), which may be written

$$
\theta_{f}=\frac{1}{\beta_{b}} \int_{0}^{\beta_{b}} \theta d \beta,
$$

with the definition for $M$,

$$
M=\frac{\beta_{b} \theta_{b}}{\beta_{b} \theta d \beta}
$$

or

$$
\theta_{b}=M \frac{1}{\beta_{b}} \int_{0}^{\beta_{b}} \theta d \beta
$$

it follows, for roots $M>1$, that

$$
\theta_{b}>\theta f
$$

indicating an inversion at the top of the convective layer. I have been unable to carry out a general discussion of the possible roots to equation (19c) but in special, representative cases it may be shown that such roots, if they exist, must correspond to entropy minima or secondary maxima. 
Thus we find the obvious result that the convective overturning never is brought to a halt in a region where the potential temperature still drops with elevation, but necessarily must extend into the upper, stable atmosphere, where the potential temperature increases upward.

It is evident from (13) that the final potential temperature will be given by

$$
\theta_{f}=\frac{1}{\beta_{b}} \int_{0}^{\beta_{b}} \theta d \beta
$$

or, if (25) is considered,

$$
\theta_{f}=\theta_{b}
$$

This result means that there is a continuous transition in temperature from the convective layer to the upper, undisturbed air column. This result does not hold if the presence of moisture in the atmosphere is taken into consideration, as will be shown below.

It is obviously impossible to treat the effect of water vapor in an exact fashion, since the amount of heat released through condensation will depend upon the particular way in which the overturning takes place. The solution given below is therefore not rigid.

Let us assume at first that the air column is not very far from saturation. If the latent heat is released through pseudoadiabatic processes similar to those described in chapter I, the total energy is given, at least approximately, by the expression

$$
E=\frac{c_{p}}{g} \int_{p b}^{p_{0}} T_{E} d p=\frac{c_{p} p_{o}}{g} \int_{x_{b}}^{1} T_{E} d x
$$

that is, the total energy of a moist air column may be computed by the same formula as that of a corresponding dry column provided we insert the equivalent temperature instead of the actual temperature. The expression (30) may be obtained by adding the total heat of condensation,

$$
\int_{0}^{b} L q \rho d z=\frac{1}{g} \int_{p b}^{p_{0}} L w d p
$$

to the total energy given by (4), making use of the fact that, approximately,

$$
T+\frac{L w}{c_{p}}=T_{E}
$$

If the entropy of liquid water be neglected the total entropy of the moist air column is given by

$$
S=\int_{0}^{b} c_{p} \log \theta_{E} \cdot \rho d z=\frac{c_{p}}{g} \int_{p_{b}}^{p_{o}} \log \theta_{E} \cdot d p=\frac{c_{p} p_{o}}{g} \int_{x_{b}}^{1} \log \theta_{E} \cdot d x .
$$

Thus also the expression for the entropy agrees formally with the corresponding formula in a dry air column, the only difference being that we now have to introduce the equivalentpotential temperature in place of the ordinary potential temperature. From the fact that 
the relation between equivalent temperature and equivalent-potential temperature is identical with the relation between temperature and potential temperature (I, 30), it follows that the problem may be solved by exactly the same method as when no moisture is present, provided the temperature distribution is assumed to be given by $T_{E}$ instead of by $T$.

If the relative humidity of the air column is low, the solution will require several steps. At first the dry-adiabatic overturning at the air column should be investigated. If the lowest temperature obtained during this overturning (presumably the end temperature at the top of the convective layer) still exceeds the highest temperature at which any adiabatically expanded particle of the original column would reach condensation, it is reasonable to assume that the latent heat will not be called into play. If the preceding condition is not fulfilled we may assume that during the convective process some strata will reach condensation and that their latent heat thus will be made available for convection. We then have to attack the problem anew, this time by means of the equivalent temperature but otherwise proceeding in the way indicated above.

To obtain the actual temperature distribution after equilibrium has been reached, we may assume that during the convective upheaval no appreciably lower temperatures occur than in the final stage and that a minimum amount of water vapor condenses. Then the final air column barely reaches saturation at its upper boundary. Since we know the pressure at this level we are in a position to calculate the water vapor content $w$ and the partial potential temperature $\theta_{d}$ at this point. As a result of the stirring, both of the above quantities must be constant all the way down to the ground, thereby also satisfying the condition that in the final stage the equivalent-potential temperature remains constant throughout. The actual determination of $w$ and $\theta_{d}$ at the top of the convective layer is best done by means of the equivalent-potential temperature diagram in Plate I. Since the pressure $\left(p_{b}\right)$ at the upper boundary is known and the water vapor is saturated at this point, we can find $w$ and $\theta_{d}$ by determining the point of intersection between the isobar $p h$ and the constant $\theta_{E}$-line characteristic of the equilibrium stage. In general it may be assumed that the relative humidity is less than 100 per cent in the air immediately above the convective layer. Since the equivalent-potential temperature is continuous at the boundary between the two columns (see page 31) and the water vapor is saturated just below this point, it follows that at the same level the partial potential temperature must increase by a finite amount. Thus, in the case of convection with condensation the theory indicates that there must be a temperature inversion at the upper boundary of the convective layer and this inversion must bave such a value that the equivalent-potential temperature and therefore also the equivalent temperature remains continuous.

To facilitate the practical application of the preceding results a special chart was constructed with the potential temperature (or equivalent-potential temperature) as ordinate and $\beta$ (see equation 20) as linear abscissa (figure 4). On the latter axis distances corresponding to $x=1.00,0.99,0.98$, etc., were set off. Thus it is possible to plot $\theta$ as a function of $\beta$, if we know the distribution of $\theta$, the potential temperature, with pressure $\left(x=\frac{p}{p_{0}}\right)$. In the typical case of an atmosphere which is unstable in the surface layers and stable at higher elevations, $\frac{d \theta}{d \beta}$ is negative for small values of $\beta$, then gradually reaches zero and finally becomes positive for large values of $\beta$. 
To obtain the solution $M=1$ we may write equation (25) in the form

$$
\beta_{b} \theta_{b}=\int_{0}^{\beta_{b}} \theta d \beta .
$$

The left member represents the area of a rectangle with $\beta_{b}$ and $\theta_{b}$ as sides. The right side measures the area enclosed by the observed curve, the $\beta$-axis and the two vertical lines $\beta=0$ and $\beta=\beta_{b}$. Thus, to obtain the desired solution, we must draw a horizontal line in such a fashion that the two areas $A B C A$ and $C D E C$ equal each other. The point $E$ obviously represents the upper boundary of the convective layer. The procedure outlined above remains the same also when the plotted curve represents the equivalent-potential temperature distribution.

It is as yet impossible to say whether the theory developed in this chapter leads to a useful criterion for the occurrence or non-occurrence of thunderstorms. Any such criterion would have to be derived statistically. Among quantities which conceivably may be correlated with the occurrence of thunderstorms, one of the most promising would seem to be the difference between the total amount of water vapor in the theoretically determined convective stage and the amount of water vapor contained within the corresponding part of the original air column. As yet no such statistical study has been made, partly because of lack of time and partly because of the lack of suitable material. The preceding discussion, then, does not pretend to offer any criterion of a higher degree of reliability than the thermodynamic diagrams already in use, but it points out a method by means of which a dependable criterion possibly may be derived.

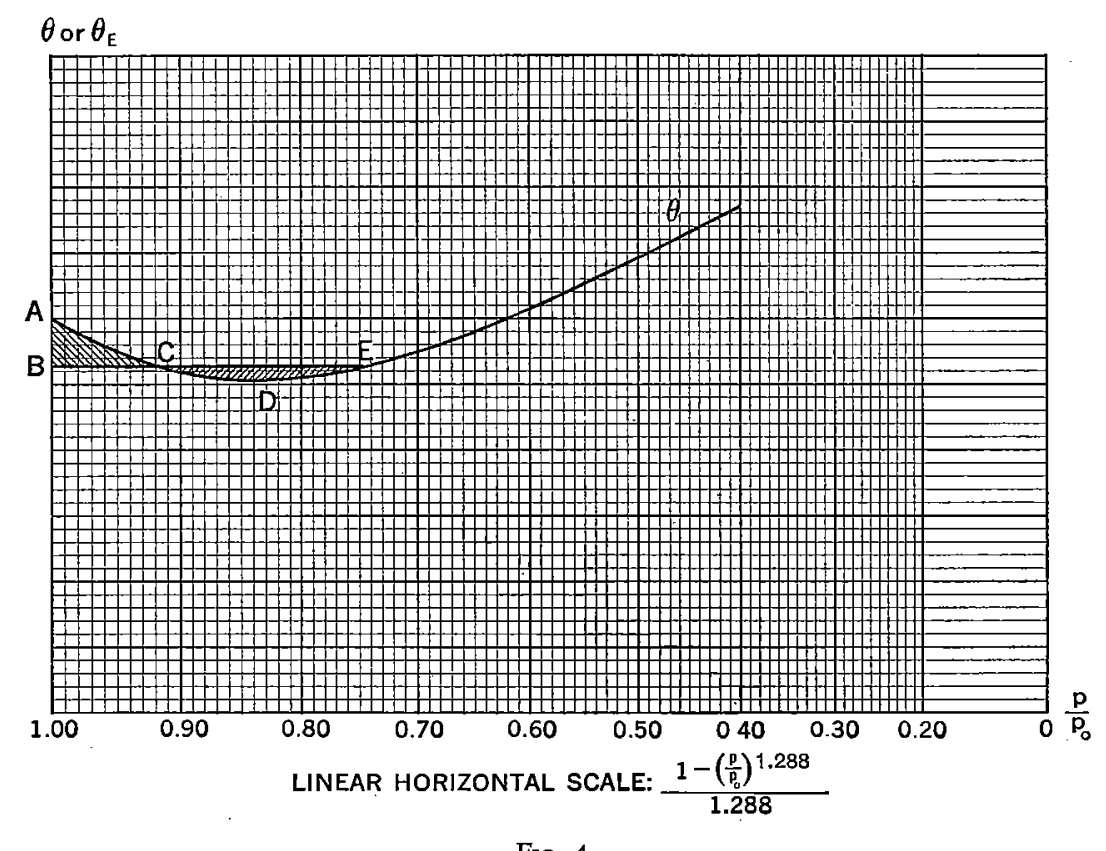

FIG. 4 


\section{Chapter III}

\section{ON THE USE OF CHARACTERISTIC CURVES IN AIR MASS ANALYSIS}

$I^{\prime}$ $\mathbf{N}$ THE first chapter some of the properties of the characteristic curve were derived from purely theoretical considerations. Whenever possible these properties were stated in non-mathematical terms. Below a small number of synoptic charts for North America will be discussed in an effort to verify the results of the preceding theoretical treatment. These charts, reproduced in Plates II to VIII, were selected from the daily working charts of the Massachusetts Institute of Technology. The original analysis by Dr. H. C. Willett has been changed only in a few minor details. To save space, morning charts only ( 8 a.m. 75th Meridian Time) have been included.

On each plate is reproduced an equivalent-potential temperature diagram, showing the characteristic curves at the various aerological stations during the period under discussion. The individual stations are indicated by their initials and the date of the sounding by a subscript. The following initials are used:

\begin{tabular}{|c|c|c|}
\hline$B$ for Broken Arrow & $D$ for Due West & $E$ for Ellendale \\
\hline$G$ for Groesbeck & $R$ & enter \\
\hline$S$ for Seattle & $S D$ & Diego \\
\hline
\end{tabular}

The location of each station is indicated on the synoptic charts by the symbol $\otimes$ and the initial of the station.

Practically all the characteristic curves are based on morning soundings, usually made around 7 or 8 a.m. Eastern Standard Time. Exceptions are indicated by the letters p.m. after the subscript. Ascents only are used.

Plate IIa (December 12, 1929) shows a well-marked warm sector of Gulf air ( $T_{g}$-air) extending from the Gulf Coast north and northeastward towards the southern part of Illinois. The warm sector proper is characterized by an almost constant temperature of $64^{\circ} \mathrm{F}$. It is surrounded by transitional air masses of continental (Canadian) origin, which have a fairly steep temperature gradient northward.

On the following day the warm sector has advanced further towards the northeast through Indiana and Ohio. It is partly encircling a stagnant body of transitional polar air over the Southeastern states. The northern and western boundary of the advancing current of Gulf air is well marked by a strong temperature discontinuity, but the southeastern boundary, in a region of very slight air movement, is less distinct.

During these two days the Gulf air passes over the stations at Groesbeck, Broken Arrow and Royal Center. It is reasonable to assume that the subsiding remnant of transitional air over the Southeast occupies only a shallow layer next to the ground and that the Gulf air therefore should be found at high levels along the Middle Atlantic Coast.

The equivalent-potential temperature diagram brings out the great similarity between the characteristic curves of Groesbeck for the two days under discussion and the curve of Broken Arrow for the thirteenth. No sounding was obtained at the latter station on the twelfth. All three curves indicate an exceedingly high moisture content at the surface (around 13 grams), decreasing rapidly with altitude. All curves show a high equivalent- 
potential temperature which decreases slightly with elevation (convective instability). The decrease in water vapor content is not uniform but interrupted by slight increases. A study of the original data reveals that these increases coincide with slight temperature inversions. These inversions may be interpreted as remnants of old fronts or as the result of a relative motion of different strata of Gulf air, one layer slowly sliding up over the one next below. There are not enough data available for us to make a decision between these explanations.

On the twelfth, Royal Center was still in the transitional air but fairly close to the warm front. This is also indicated by the characteristic curve $\left(R_{12}\right)$, which is clearly $V$-shaped. The moisture content increases from about 5.4 grams at the ground to a maximum of about 9.8 grams. The upper part of the characteristic curve shows the same convective instability (upward decreasing equivalent-potential temperature) that was found in the curves from Groesbeck and Broken Arrow. At the point of maximum water vapor content ( $470 \mathrm{~m}$. above ground) the equivalent-potential temperature is about $319.5^{\circ} \mathrm{A}$ or only slightly less than the corresponding values within the warm sector itself $\left(320^{\circ}-330^{\circ} \mathrm{A}\right)$. Thus it is reasonable to assume that the sounding at Royal Center actually penetrates the warm front and that the upper part of the characteristic curve represents Gulf air. The water vapor content at the ground ( 5.4 grams) is slightly higher than what might normally be expected in transitional continental air; it may be attributed to evaporation from rain which has previously fallen through the thin wedge of transitional air. The original record indicates that throughout the flight the wind at the surface remains light and southeasterly. At $470 \mathrm{~m}$. the wind is SW $12.9 \mathrm{~m} . \mathrm{p} . \mathrm{s}$. Dense fog and mist prevail. Thus there can be little doubt that the ascent penetrates the warm front.

On the thirteenth the map indicates that the boundary of the Gulf current must be in the immediate vicinity of Royal Center. This is verified by the characteristic curve $\left(R_{13}\right)$ in which the wedge of transitional air has disappeared almost completely. The maximum water vapor content is about 10.4 grams and the equivalent-potential temperature at this point is $322^{\circ}$. The upper part of the characteristic curve is practically identical with the curve for the preceding day. Both curves show a slightly lower equivalent-potential temperature than the soundings taken in the warm sector proper. The surface wind is SW 4 m.p.s.

The sounding for Due West for the thirteenth has a characteristic curve $\left(D_{13}\right)$ quite similar to that for Royal Center the day before. The moisture content is fairly low at the surface ( 5.6 grams) and then increases to a maximum of 9.5 grams (at $590 \mathrm{~m}$. above ground). At this point the equivalent-potential temperature is about $318.5^{\circ} \mathrm{A}$, indicating the presence of Gulf air. This conclusion is supported by the fact that the temperature increases from $5.9^{\circ} \mathrm{C}$ at the ground to $13.3^{\circ} \mathrm{C}$ at $590 \mathrm{~m}$. The upper part of the characteristic curve has the typical $\theta_{d}$ - and $w$ - values of the Gulf air in the warm sector itself, but by this time the warm current seems to have lost its characteristic convective instability, since the curve now closely parallels the pseudoadiabatic line $320^{\circ} \mathrm{A}$. This change is probably the result of increased stirring as the air current passes over the mountainous region in the southeast. The wind is SW to WSW at all levels.

The characteristic curves obtained for the Gulf air during this period agree well with the curves given by Earl and Turner. ${ }^{15}$

\footnotetext{
${ }^{15}$ Earl and Turner, op. cit. in fn. 1.
} 
Plates III and IV represent the synoptic situation December 16-19, 1929. During this period a current of Gulf air moves northward along the Mississippi River, then gradually bends northeastward in a narrow stream. Its advance is checked by a body of transitional air along the Atlantic Coast. Finally a strong and deep current of fresh continental polar air $\left(P_{c}\right.$-air $)$ invades the Mississippi Valley and gradually pushes the Gulf air off the continent.

The equivalent-potential temperature diagrams for the same period are reproduced in the plates. The characteristic curves from Groesbeck for the 16th and 17th show high moisture content at the surface (between 12 and 13 grams) and convective instability. The curve for Due West on the 18th, when the $T_{g}$-air has reached the Atlantic coast, is quite similar except that the convective instability now has been somewhat reduced. In this case both ascent (a) and descent (b) were plotted. The morning chart for this date (December 18) still seems to indicate the presence of a shallow layer of transitional air next to the ground at Due West. However, at the time of the descent (10 a.m.-11 a.m.) this layer apparently has been washed away by the Gulf air, as no trace of it is found in the characteristic curve.

On the eighteenth, Royal Center is obviously located in the advancing polar air current but not very far from the northern boundary of the warm sector where warm air is advancing at higher levels. The characteristic curve $\left(R_{18}\right)$ shows an increase of the water vapor content at high levels but apparently the sounding does not penetrate the warm front. The original record carries the following note. "10 Nb. NNE throughout flight. Altitude of Nb. base about $550 \mathrm{~m}$. at $7.28 \mathrm{a} . \mathrm{m}$. and increased to about $700 \mathrm{~m}$. at 9.04 a.m." The top elevation reached during the flight was $1860 \mathrm{~m}$. above ground. At this elevation the relative humidity was 100 per cent and the wind direction was indicated as NNE.

Broken Arrow and Groesbeck are now submerged in the polar air and their characteristic curves present striking evidence to this effect. The moisture content at the surface is between one and two grams and the curves are almost vertical.

The characteristic curves for the 19th are typical of fresh continental polar air in winter time. The water vapor content is less than one gram, and the characteristic curves are vertical, demonstrating the marked stability of the continental polar air. The curve for Ellendale extends down to very low temperatures. Apparently the air is heated on its way from Ellendale to Groesbeck, but the movement is too rapid to permit much of an increase in the water vapor content, showing that the water vapor content is a far more conservative property than the potential temperature. The characteristic curves obtained for this cold wave stand in excellent agreement with similar curves presented by Earl and Turner. ${ }^{16}$

The situation presented in Plates V and VI covers the period January 7-10, 1930 and is similar to the ones already mentioned. A narrow stream of Gulf air, followed by a powerful current of continental polar air, moves slowly northeastward from the Mississippi Valley towards Lake Erie. Also in this case the advance of the warm air is checked by a stagnant body of transitional air over the southeastern part of the United States. Finally occlusion takes place and the polar air spreads eastward towards the Atlantic coast.

The characteristic curves for this period are reproduced in Plate VI. The curve

${ }^{16}$ Earl and Turner, op. cit. in fn. 1. 
from Groesbeck for the ninth is particularly interesting. At the surface the moisture content is in the vicinity of 3 grams. It then increases to a maximum value of 7.5 grams (at $975 \mathrm{~m}$. above the ground). At this point the equivalent-potential temperature is about $312^{\circ}$, which seems to approach the characteristic value for Gulf air on this occasion. According to the original record the sky remained overcast during the flight, the base of the stratus cloud deck being about $425 \mathrm{~m}$. above the ground. The surface wind was NNW at the surface and NW at the top of the flight. Thus it would seem that the ascent barely missed reaching into the warm air.

The sounding at Broken Arrow on the same day also shows an increase of moisture content with elevation. From the synoptic chart it may be seen that Broken. Arrow is too far from the front for the sounding to reach into the warm air. The water vapor content at the ground is typical of a fresh continental polar current at this latitude. The equivalent-potential temperature at the top of the flight $(1470 \mathrm{~m}$. above ground) is about $293^{\circ}$, which is considerably below the values characteristic of the Gulf air. At this level the wind direction is WNW, while the surface wind is N. A study of the original record indicates the existence of a strong temperature inversion within the cold air $\left(-13.3^{\circ} \mathrm{C}\right.$ at $370 \mathrm{~m}$. above ground, $-1.1^{\circ} \mathrm{C}$ at $1100 \mathrm{~m}$.). Since this inversion is accompanied by an increase in water vapor content, one might easily be inclined to mistake it for the boundary between polar and Gulf air. The equivalent-potential temperatures demonstrate very definitely that such is not the case, but that the inversion must be due to relative motion of different strata of polar air (secondary front). The existence of secondary fronts is indicated also by the strong northward temperature gradient at the surface within the polar air.

The sounding at Due West for the eighth indicates the existence of transitional air at the surface, with typical Gulf air above and this conclusion is verified by the original record. At 8.26 a.m. the surface wind is $S E$, the wind at $470 \mathrm{~m}$. above the ground SSW. At the end of the flight $(10.47 \mathrm{a} . \mathrm{m}$.) the surface wind has changed to SW. This change in wind direction would seem to indicate that the Gulf air has reached the surface, but this conclusion is contradicted by the value of the water vapor content at the surface (about 9.3 grams) which is somewhat too low for Gulf air proper at the surface. One must therefore assume that the transitional air remains over Due West. This is borne out by the characteristic curve and synoptic chart for the next day.

The curves from Ellendale and Royal Center for the tenth are typical of a fresh current of continental polar air. The curve for Due West still shows transitional air at the surface (up to an elevation of $1015 \mathrm{~m}$. above the ground) and Gulf air above. At Groesbeck the surface air is plainly of polar origin. At $1150 \mathrm{~m}$. above the ground the wind is easterly and the water vapor content increases to 3.76 grams. This high value is probably due to previous absorption of moisture from precipitation falling through the cold wedge.

The period February 23-26 (Plates VII and VIII) represents another example of Gulf air invading the Mississippi Valley, but this time on a larger scale than in any of the previous examples. On the twenty-third the warm air covers the entire Gulf coast of the United States with the exception of Florida and extends northeastward as far as Louisville, Ky. The following day the warm sector, advancing slowly northward, has reached Springfield, Ill. On the twenty-fifth, the warm air extends to Cleveland northward and Washington, D.C., eastward. Finally, on the twenty-sixth, a current of Pacific polar air gradually forces the Gulf air eastward out over the ocean. On this day the boundary between the 
Gulf air and the polar air extends from the Mississippi Delta towards the southern part of Ohio.

It is particularly noteworthy that during this development no stagnation of transitional air takes place over the Southeast as in the three preceding cases, but the Gulf air is permitted to advance over a very broad front finally covering all the southeastern states.

The characteristic curves for this period show convective instability at all stations within the warm sector. The curve from Due West for the twenty-fifth indicates that there is still a layer of transitional air next to the ground. The original record shows an inversion with a temperature increase from $11.6^{\circ} \mathrm{C}$ at the ground to $17.2^{\circ} \mathrm{C}$ at $350 \mathrm{~m}$. elevation.

The curve from Groesbeck for the twenty-fifth deserves particular attention. It shows an almost constant potential temperature up to the second point $(290 \mathrm{~m}$. above the ground), from then on a constant equivalent-potential temperature of about $303^{\circ}$ and finally, from $780 \mathrm{~m}$. to $1420 \mathrm{~m}$. above the ground, a strong increase in moisture content and equivalent-potential temperature. The middle part agrees fairly well with the curve from the same station for the following day (Pacific polar air) whereas the surface and top points have values fairly characteristic of Gulf air. The synoptic chart shows that Groesbeck is located in the immediate vicinity of the front between the Gulf air and a small body of fairly warm transitional Pacific polar air. It seems probable that the upper part of the curve indicates a boundary between Pacific polar air and over-running Gulf air, but this Pacific air does not extend down to the ground as may be seen from the high water vapor content at the surface. One must therefore conclude that the transitional Pacific air advances over a thin layer of Gulf air. This view is supported by the observed wind directions. At the surface the wind is W $7.2 \mathrm{~m} . p . s$., at $360 \mathrm{~m}$. above the ground it is WNW $10.2 \mathrm{~m}$.p.s. It then shifts to NNW $6.1 \mathrm{~m}$.p.s. at $780 \mathrm{~m}$. and NNW $5.7 \mathrm{~m} . p . s$. at $860 \mathrm{~m}$. above the ground. At still higher elevations the wind backs to $\mathrm{NW}$, having the value NW $3.0 \mathrm{~m}$.p.s. at $1420 \mathrm{~m}$.

Broken Arrow on the same occasion is close to the boundary between the Gulf air and the Pacific polar air. The characteristic curve plainly indicates Gulf air. The absence of over-running in this case probably should be ascribed to the greater difference in temperature between the Gulf air and the fresh Pacific polar air.

The curve of Royal Center for the twenty-sixth is fairly characteristic of Pacific polar air. The surface water vapor content is generally around four or five grams and the characteristic curve roughly parallel to the pseudoadiabatic lines. Unfortunately there is no morning ascent at Broken Arrow on this day; with the few ascents at hand on this occasion it is impossible to study the properties of the Pacific air in detail.

During the ninth and tenth of March, 1930, a strong current of Pacific polar air invaded the United States and gradually spread eastward, reaching in turn every aerological station of the country. Part of the Pacific air current returned westward over the California coast but the greater part of the current continued eastward towards the Atlantic coast. This current was not homogeneous, but consisted of several different bodies of Pacific air, separated by weak, occluded fronts. The scantiness of observations from the western part of the country and the profoundly disturbing influence of the mountains make it impossible to carry out a detailed analysis, and to separate the different bodies of Pacific polar air. For this reason the synoptic charts for the period in question have not been reproduced. However, due to the maritime history of this air current, it is probably permissible to disregard such differences as may have existed between 
its various parts and consider it as a fairly homogeneous Pacific polar air current. The characteristic curves are reproduced in figure 5 . The agreement between the different curves is striking, especially if the great difference in time and distance between soundings is considered. Judging from this case, the Pacific polar air is much richer in water vapor than the Canadian polar air, which is to be expected in view of its maritime origin and higher temperature. The same result was obtained by Earl and Turner.

While the Canadian polar air during the winter season always is highly stable, the characteristic curves for the Pacific polar air seem to run fairly parallel to the pseudoadiabatic lines, indicating a thorough stirring of the air. Additional material will be presented below to strengthen this conclusion.

It is instructive to notice the differences in stability between the three principal air masses discussed above.

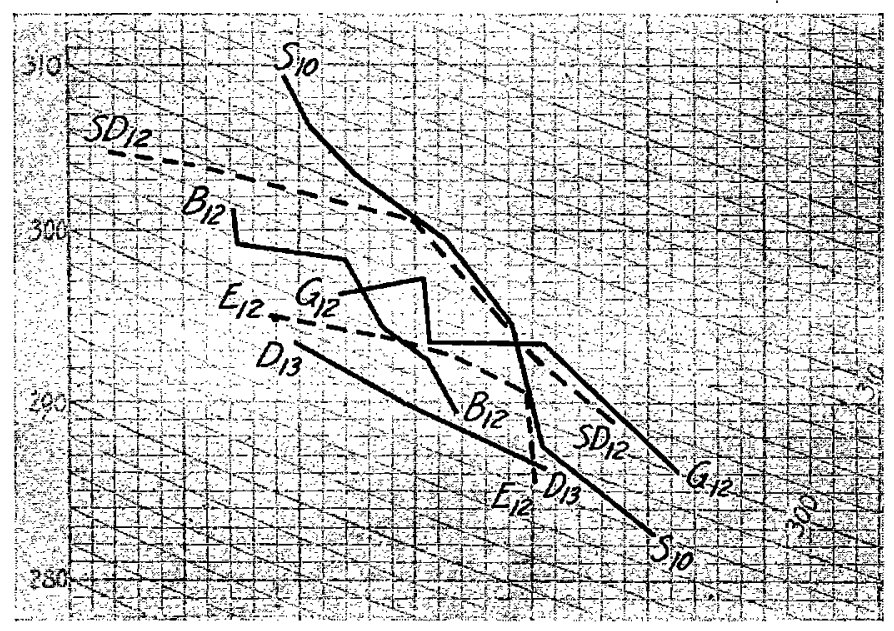

FIG. 5.

The currents of continental polar air over the central part of the United States have travelled only a short distance from the region in which their predominant characteristics were acquired, namely the extreme northern part of Canada. Similarly, the Gulf air observed in the Mississippi Valley probably acquired its most important properties only five hundred or a thousand miles further south. It is probable that the changes wrought in those air masses during so short a journey from their source are fairly insignificant except in the surface strata. Therefore, the temperature and moisture distribution within these two types of air masses is determined primarily by the meteorological conditions prevailing at their sources, i.e., over the Gulf of Mexico and in the northern part of Canada. It is especially the intensity of the solar radiation, the surface temperature of the Gulf and the area and the temperature of the snow cover in northern Canada which determine the properties of the Gulf air and the polar air at their sources and, in view of the foregoing, also in the United States. This explains the dryness and the pronounced stability of the polar air and also the high vapor content and convective instability of the Gulf air. 
The Pacific polar air, on the other hand, has travelled thousands of miles across the ocean from its source (Siberia or the Arctic Sea). During this long journey it has been heated from below, absorbed moisture and gradually reached convective equilibrium. This condition, typical also of the maritime polar air of Northwestern Europe, ${ }^{17}$ is strikingly illustrated by Plates IX and X, in which the equivalent-potential temperatures over San Diego and Seattle have been plotted against elevation for each day during several months. It is evident that at both these stations the equivalent-potential temperature remains fairly constant with elevation. In view of the preceding discussion this result is reasonable enough when Seattle is considered. At San Diego, however, the air motion is much more sluggish and a strong inversion with humidity decrease often separates a westerly surface current from a strong easterly current above. Under those circumstances it is remarkable that the decrease in water vapor content and the increase in potential temperature should be such as to make the equivalent-potential temperature practically constant with elevation. This would seem to indicate that the surface air and the upper current must be of the same origin and that they have described nearly identical paths across the ocean but eventually reach San Diego by slightly different routes, the surface air moving down along the coast while the upper strata make a detour over the continent, eventually reaching Southern California from the east or northeast.

The corresponding curves for February, 1930, from Ellendale, Broken Arrow, Royal Center and Groesbeck (Plate XI) have a very different appearance. At Ellendale the equivalent-potential temperature increases in an astonishingly regular way with elevation, indicating pronounced stability. The same is true with respect to most of the curves from Broken Arrow, although a few show marked convective instability. At Groesbeck the proportion of stable to unstable curves is more even.

If the curves for Seattle and Groesbeck are compared it is seen that at Seattle the individual curves are all practically alike, whereas at Groesbeck the curves vary greatly from day to day. Considering the fact that Groesbeck must be regarded as a fairly maritime station, it becomes evident that the continentality in itself does not determine the distribution of the equivalent-potential temperature with elevation. The continentality simply determines. the frequency of the occurrence of different types of air masses, and these variations in frequency are reflected in the equivalent-potential temperature curves. Between northern Canada (source of the $P_{c}$-air) and the Gulf of Mexico (source of the $T_{g}$-air) there is a distance of about two thousand miles only. Groesbeck, located between the two, is exposed to frequent invasions of air from both sources, which accounts for the extreme variability of its equivalent-potential temperatures.

It may be concluded that air mass analysis on the West Coast of North America is beset with great difficulties, since the individual air masses, due to long travel over the ocean, have become thoroughly modified and, therefore, differ very slightly from one another (the only exception being some very infrequent outbreaks of Canadian polar air which cross the mountains and reach the Pacific). This conclusion has been borne out also by a synoptic investigation of the weather phenomena of California. ${ }^{18}$

In the Middle West and to a lesser degree along the Atlantic seaboard, two of the principal air masses, Gulf air and Canadian air, often have just left their source; their

17 Troeger, H., Ein konservatives meteorologisches Element, Annalen der Hydrographie und maritimen Meteorologit, Vol. 58, p. 358, Berlin, 1930.

${ }^{18}$ Byers, H. R., Characteristic Weather Phenomena of California, Massacbusetts Institute of Tecbnology Meteorological Papers, Vol. I, No. 2, Cambridge, Mass., 1931. 
characteristic properties are, therefore, determined by the meteorological conditions in the region where they originate. Since these regions are so utterly different, it is obvious that air mass analysis by the methods here outlined must be far more successful when applied to that part of the continent which is located east of the Rocky Mountains than when applied to the Pacific Coast states. It is true that the air masses of the Middle West, on account of their continental paths, may vary greatly in their surface characteristics from day to day, more, perhaps, than one and the same type of Pacific air would vary, but the sources of the principal Middle West air masses differ so fundamentally between themselves that there can be no difficulty in classifying these air masses. This is not true with regard to the West Coast, where, on account of their length, the trajectories mean almost everything and the sources very little in determining the characteristics of the air masses, and where, therefore, the differences between the several types of air currents are very small, even though they may be fairly consistent.

The conclusions formulated above stand in sharp contrast to the ideas set forth by $\mathrm{R}$. H. Weightman in a recent publication. ${ }^{19}$ Weightman writes: "It is doubted by those who have considered the situation that the same advantageous results as in western Europe can be obtained from air mass analysis over areas where predominatingly continental climate obtains. However, such analysis would undoubtedly be profitable along our western coasts." Weightman accepts the Polar front theory as a fairly accurate description of the structure of extratropical cyclones. This theory necessarily presupposes the existence of well-defined air masses, distinguisbable by certain dynamically significant conservative or nearly conservative properties. Otherwise little meaning could be attached to the fronts. Air mass analysis developed as a substitute for front analysis with the growing realization that at the ground fronts often may be obscured by a number of disturbing influences. Air mass analysis may become difficult when the majority of the cyclones considered are occluded and the trajectories of the air masses involved are very long. This, however, is not the case in the central and eastern parts of the United States since, according to the same writer, "between 40 and 50 per cent of our LOWS develop over the continent, or waters contiguous thereto." The LOWS of the Pacific coast, on the other hand, are nearly all occluded. 
IV. TABLES AND PLATES. 
TABLE A

Pressure of Saturated Water Vapor (Over Plank Water Surface)

The pressures are given in $\mathrm{mm}$. $\mathrm{Hg}$.

\begin{tabular}{c|c|c|c|c|c|c|c|c|c|c}
\hline${ }^{\circ} \mathbf{C}$ & $\mathbf{0}$ & $\mathbf{1}$ & $\mathbf{2}$ & $\mathbf{3}$ & $\mathbf{4}$ & $\mathbf{5}$ & $\mathbf{6}$ & $\mathbf{7}$ & $\mathbf{8}$ & 9 \\
\hline+20 & 17.535 & $\mathbf{1 8 . 6 5 0}$ & 19.827 & 21.068 & 22.377 & 23.756 & 25.209 & 26.739 & 28.349 & 30.043 \\
\hline+10 & 9.209 & 9.844 & 10.518 & 11.231 & 11.987 & 12.788 & 13.634 & 14.530 & 15.477 & 16.477 \\
\hline+0 & 4.579 & 4.926 & 5.294 & 5.685 & 6.101 & 6.543 & 7.013 & 7.513 & 8.045 & 8.609 \\
\hline-0 & 4.579 & 4.256 & 3.952 & 3.669 & 3.404 & 3.158 & 2.928 & 2.712 & 2.509 & 2.320 \\
\hline-10 & 2.143 & 1.979 & 1.826 & 1.684 & 1.551 & 1.429 & 1.315 & 1.208 & 1.109 & 1.018 \\
\hline-20 & 0.934 & 0.856 & 0.783 & 0.716 & 0.654 & 0.596 & 0.543 & 0.494 & 0.449 & 0.408 \\
\hline-30 & 0.371 & 0.337 & 0.307 & 0.279 & 0.253 & 0.230 & 0.208 & 0.188 & 0.170 & 0.154 \\
\hline-40 & 0.139 & 0.125 & 0.113 & 0.102 & 0.092 & 0.083 & 0.075 & 0.067 & 0.060 & 0.054 \\
\hline \hline
\end{tabular}

The above values are taken from M. Robitzsch : Die Verwertung der durch aerologische Vetsuche gewonnenen Feuchtigkeitsdaten zur Diagnose der jeweiligen atmosphärischen Zustände, Die Arbeiten des Preussischen Aeronautischen Observatoriums XVI. Band, Wissenschaftliche Abhandlungen, Heft C, Braunschweig, 1928.

TABLE B

Partial Potrential Temperaturs Correctron

Add the tabulated quantities to the potential temperature in order to obtain the partial potential temperature $(\Delta \theta d=\theta d-\theta)$

\begin{tabular}{|c|c|c|c|c|c|c|c|c|c|c|c|}
\hline $\boldsymbol{w} \theta$ & 253 & 263 & 273 & 283 & 293 & 303 & 313 & 323 & 333 & 343 & 353 \\
\hline o & 0.0 & 0.0 & 0.0 & 0.0 & 0.0 & 0.0 & 0.0 & 0.0 & 0.0 & 0.0 & 0.0 \\
\hline 1 & 0.1 & 0.1 & 0.1 & 0.1 & 0.1 & 0.1 & 0.1 & 0.1 & 0.2 & 0.2 & 0.2 \\
\hline 2 & 0.2 & 0.2 & 0.3 & 0.3 & 0.3 & 0.3 & 0.3 & 0.3 & 0.3 & 0.3 & 0.3 \\
\hline 3 & & 0.4 & 0.4 & 0.4 & 0.4 & 0.4 & 0.4 & 0.4 & 0.5 & 0.5 & 0.5 \\
\hline 4 & & 0.5 & 0.5 & 0.5 & 0.5 & 0.6 & 0.6 & 0.6 & 0.6 & 0.6 & 0.7 \\
\hline 5 & & 0.6 & 0.6 & 0.7 & 0.7 & 0.7 & 0.7 & 0.7 & 0.8 & 0.8 & 0.8 \\
\hline 6 & & & 0.8 & 0.8 & 0.8 & 0.8 & 0.9 & 0.9 & 0.9 & 1.0 & 1.0 \\
\hline 7 & & & 0.9 & 0.9 & 0.9 & 1.0 & 1.0 & 1.0 & 1.1 & 1.1 & 1.1 \\
\hline 8 & & & 1.0 & 1.0 & 1.1 & 1.1 & 1.2 & 1.2 & 1.2 & 1.3 & 1.3 \\
\hline 9 & & & 1.1 & 1.2 & 1.2 & 1.3 & 1.3 & 1.3 & 1.4 & 1.4 & 1.5 \\
\hline 10 & & & 1.3 & 1.3 & 1.3 & 1.4 & 1.4 & 1.5 & 1.5 & 1.5 & 1.6 \\
\hline 11 & & & 1.4 & 1.4 & 1.5 & 1.5 & 1.6 & 1.6 & 1.7 & 1.7 & 1.8 \\
\hline 12 & & & & 1.6 & 1.6 & 1.7 & 1.7 & 1.8 & 1.8 & 1.9 & 1.9 \\
\hline 13 & & & & 1.7 & 1.7 & 1.8 & 1.9 & 1.9 & 2.0 & 2.0 & 2.1 \\
\hline 14 & & & & 1.8 & 1.9 & 1.9 & 2.0 & 2.1 & 2.1 & 2.2 & 2.3 \\
\hline 15 & & & & 1.9 & 2.0 & 2.1 & 2.2 & 2.2 & 2.3 & 2.4 & 2.4 \\
\hline 16 & & & & 2.1 & 2.1 & 2.2 & 2.3 & 2.4 & 2.4 & 2.5 & 2.6 \\
\hline 17 & & & & 2.2 & 2.3 & 2.4 & 2.4 & 2.5 & 2.6 & 2.7 & 2.7 \\
\hline 18 & & & & 2.3 & 2.4 & 2.5 & 2.6 & 2.7 & 2.7 & 2.8 & 2.9 \\
\hline 19 & & & & 2.5 & 2.5 & 2.6 & 2.7 & 2.8 & 2.9 & 3.0 & 3.1 \\
\hline 20 & & & & 2.6 & 2.7 & 2.8 & 2.9 & 2.9 & 3.0 & 3.1 & 3.2 \\
\hline
\end{tabular}


TABLE C

Equivalent-Potential Temperature Correction

Add the tabulated quantities to the partial potential temperature in order to obtain the equivalent-potential temperature $(\Delta \theta E=\theta E-\theta d)$

\begin{tabular}{|c|c|c|c|c|c|c|c|c|c|c|c|c|c|c|c|c|c|c|c|c|c|}
\hline d & 248 & 253 & 258 & 263 & 268 & 273 & 278 & 283 & 288 & 293 & 298 & 303 & 308 & 313 & 318 & 323 & 328 & 333 & 338 & 343 & 348 \\
\hline .0 & 0.0 & 0.0 & 0.0 & 0.0 & 0.0 & 0.0 & 0.0 & 0.0 & 0.0 & 0.0 & 0.0 & 0.0 & 0.0 & 0.0 & 0.0 & 0.0 & 0.0 & 0.0 & 0.0 & 0.0 & 0. \\
\hline .2 & 0.5 & 0.6 & 0.6 & 0.6 & 0.6 & 0.6 & 0.6 & 0.6 & $\begin{array}{l}5 \\
\end{array}$ & 0.7 & 0.7 & 0.7 & 0.7 & 0.7 & 0.7 & 0.8 & 0.8 & 0.8 & 0.8 & 0.8 & 0. \\
\hline 4 & 1.0 & 1.1 & 1.1 & 1.1 & 1.2 & 1.2 & 1.2 & 1.2 & 1.3 & 1.3 & 1.3 & 1.3 & 1.4 & 1.4 & 1.4 & 1.5 & 1.5 & 1.5 & I.5 & 1.5 & 1. \\
\hline 6 & 1.5 & 1.6 & 1.6 & 1.7 & 1.7 & 1.7 & 1.8 & 1.8 & \begin{tabular}{|l|}
3 \\
\end{tabular} & 1.9 & 1.9 & 2.0 & 2.0 & 2.0 & 2.1 & 2.1 & 2.2 & 2.2 & 2.2 & 2.3 & 2.3 \\
\hline 8 & 2.0 & 2.1 & 2.1 & 2.2 & 2.2 & 2.3 & 2.3 & 2.4 & $\begin{array}{l}4.4 \\
\end{array}$ & 2.5 & 2.5 & 2.6 & 2.6 & 2.7 & 2.7 & 2.8 & 2.8 & 2.9 & 2.9 & 3.0 & 3.0 \\
\hline 0 & 2.5 & 2.5 & 2.6 & 2.7 & 2.7 & 2.8 & 2.9 & 2.9 & 3.0 & 3.0 & 3.1 & 3.2 & 3.2 & 3.3 & 3.4 & 3.4 & 3.5 & 3.6 & 3.6 & 3.7 & 3.8 \\
\hline 2 & & 3.0 & 3.1 & 3.2 & 3.2 & 3.3 & 3.4 & 3.5 & 3.5 & 3.6 & 3.7 & 3.8 & 3.8 & 3.9 & 4.0 & 4.1 & 4.2 & 4.2 & 4.3 & 4.4 & 4.5 \\
\hline 4 & & 3.5 & 3.6 & 3.7 & 3.7 & 3.8 & 3.9 & 4.0 & 4.1 & 4.2 & 4.3 & 4.3 & 4.4 & 4.5 & 4.6 & 4.7 & 4.8 & 4.9 & 5.0 & .1 & 5.2 \\
\hline 1.6 & & 3.9 & 4.0 & 4.1 & 4.2 & \begin{tabular}{|l|}
4.3 \\
\end{tabular} & 4.4 & 4.5 & 4.6 & 4.7 & 4.8 & 4.9 & 5.0 & 5.1 & 5.2 & 5.3 & 5.4 & 5.6 & 5.7 & 5.8 & 5.9 \\
\hline 8 & & & 4.5 & 4.6 & 4.7 & 4.8 & 5.0 & 5.1 & 2 & 5.3 & 5.4 & 5.5 & 5.6 & 5.7 & 5.9 & 6.0 & 6.1 & 6.2 & 6.3 & 6.4 & 6.5 \\
\hline 0 & & & 5.0 & 5.1 & 5.2 & 5.3 & 5.5 & 5.6 & 5.7 & 5.8 & 6.0 & 6.1 & 6.2 & 6.4 & 6.5 & 6.6 & 6.7 & 6.9 & 7.0 & 7.1 & 7.2 \\
\hline 5 & & & 6.1 & 3 & 6.4 & 6.6 & 6.7 & 6.9 & 7.1 & 7.2 & 7.4 & 7.5 & 7.7 & 7.8 & 8.0 & 8.1 & 8.3 & 8.5 & 8.6 & 8.7 & 8.9 \\
\hline .0 & & & & 7.4 & 7.6 & 7.8 & 8.0 & 8.2 & 4 & 8.5 & 8.7 & 8.9 & 9.1 & 9.3 & 9.4 & 9.6 & 9.8 & 10.0 & 10.2 & 10.4 & 10.6 \\
\hline 3.5 & & & & 8.6 & 8.8 & 9.0 & 9.2 & 9.4 & 9.6 & 9.9 & 10.1 & 10.3 & 10.5 & 10.7 & 10.9 & 11.2 & 11,4 & 11.6 & 11.8 & 12.0 & 12.2 \\
\hline 4.0 & & & & & 0 & 10.2 & 10.5 & 10.7 & .9 & 11.2 & 11.4 & 11.7 & 11.9 & 12.1 & 12.4 & 12.6 & 12.9 & 13.1 & 13.4 & 13.6 & 13.9 \\
\hline 4.5 & & & & & .1 & 1 & 11.7 & 11.9 & 12.2 & 12.5 & 12.8 & 13.0 & 13.3 & 13.6 & 13.9 & 14.2 & 14,4 & 14.7 & 15.0 & 15.3 & 15.6 \\
\hline
\end{tabular}


TABLE C (continued)

\begin{tabular}{|c|c|c|c|c|c|c|c|c|c|c|c|c|c|c|c|c|c|c|c|c|}
\hline$\theta_{d}$ & 271 & 273 & 275 & 277 & 279 & 281 & 283 & 285 & 287 & 289 & 291 & 293 & 295 & 297 & 299 & 301 & 303 & 305 & 307 & 309 \\
\hline 5.0 & 12.5 & 12.6 & 12.7 & 12.8 & 13.0 & 13.1 & 13.21 & 13.31 & 13.4 & 13.5 & 13.7 & 13.8 & 13.9 & 14.0 & 14.1 & 14.3 & 14.4 & 14.5 & 14.6 & 14.8 \\
\hline 5.5 & 13.6 & 13.8 & 13.9 & 14.0 & 14.2 & 14.3 & 14.41 & 14.61 & 14.7 & 14.8 & 15.0 & 15.1 & 15.2 & 15.4 & 15.5 & 15.6 & 15.7 & 15.9 & 16.0 & 16.1 \\
\hline 6.0 & & 14.9 & 15.1 & 15.2 & 15.4 & 15.5 & 15.61 & 15.81 & 15.9 & 16.1 & 16.2 & 16.4 & 16.5 & 16.7 & 16.8 & 17.0 & 17.1 & 17.2 & 17.4 & 17.5 \\
\hline 6.5 & & 16.1 & 16.2 & 16.4 & 16.6 & 16.7 & 16.91 & 17.01 & 17.2 & 17.3 & 17.5 & 17.7 & 17.8 & 18.0 & 18.1 & 18.3 & 18.4 & 18.6 & 18.8 & 18.9 \\
\hline 7.0 & & & 17.4 & 17.6 & 17.8 & 17.9 & 18.1 & 18.31 & 18.4 & 18.6 & 18.8 & 18.9 & 19.1 & 19.3 & 19.4 & 19.6 & 19.8 & 20.0 & 20.1 & 20.3 \\
\hline 7.5 & & & 18.6 & 18.8 & 18.9 & 19.1 & 19.31 & 19.51 & 19.7 & 19.8 & 20.0 & 20.2 & 20.4 & 20.6 & 20.8 & 20.9 & 21.1 & 21.3 & 21.5 & 21.6 \\
\hline 8.0 & & & & 20.0 & 20.1 & 20.3 & 20.52 & 20.72 & 20.9 & 21.1 & 21.3 & 21.5 & 21.7 & 21.9 & 22.1 & 22.2 & 22.5 & 22.6 & 22.8 & 23.0 \\
\hline 8.5 & & & & & 21.3 & 21.6 & 21.72 & 22.02 & 22.1 & 22.3 & 22.5 & 22.8 & 23.0 & 23.2 & 23.4 & 23.6 & 23.8 & 24.0 & 24.2 & 24.4 \\
\hline 9.0 & & & & & 22.5 & 22.7 & 23.0 & 23.1 & 23.4 & 23.6 & 23.8 & 24.0 & 24.2 & 24.4 & 24.7 & 24.9 & 25.1 & 25.3 & 25.5 & 25.8 \\
\hline 9.5 & & & & & & 23.9 & 24.2 & 24.4 & 24.6 & 24.8 & 25.1 & 25.3 & 25.5 & 25.7 & 26.0 & 26.2 & 26.4 & 26.7 & 26.9 & 27.1 \\
\hline 10.0 & & & & & & 25.1 & 25.4 & 25.6 & 25.9 & 26.1 & 26.3 & 26.6 & 26.8 & 27.0 & 27.3 & 27.5 & 27.8 & 28.0 & 28.2 & 28.5 \\
\hline 10.5 & & & & & & 26.3 & 26.6 & 26.8 & 27.1 & 27.3 & 27.6 & 27.8 & 28.1 & 28.3 & 28.6 & 28.8 & 29.1 & 29.3 & 29.6 & 29.8 \\
\hline 11.0 & & & & & & & 27.8 & 28.0 & 28.3 & 28.5 & 28.8 & 29.1 & 29.4 & 29.6 & 29.9 & 30.1 & 30.4 & 30.7 & 30.9 & 31.2 \\
\hline 11.5 & & & & & & & 29.0 & 29.2 & 29.5 & 29.8 & 30.1 & 30.4 & 30.6 & 30.9 & 31.2 & 31.5 & 31.7 & 32.0 & 32.3 & 32.6 \\
\hline 12.0 & & & & & & & 30.2 & 30.5 & 30.8 & 31.0 & 31.3 & 31.6 & 31.9 & 32.2 & 32.5 & 32.8 & 33.0 & 33.3 & 33.6 & 33.9 \\
\hline 12.5 & & & & & & & & 31.7 & 32.0 & 32.3 & 32.6 & 32.9 & 33.2 & 33.5 & 33.8 & 34.1 & 34.4 & 34.7 & 35.0 & 35.3 \\
\hline 13.0 & & & & & & & & 32.9 & 33.2 & 33.5 & 33.8 & 34.1 & 34.4 & 34.8 & 35.1 & 35.4 & 35.7 & 36.0 & 36.3 & 36.6 \\
\hline 13.5 & & & & & & & & 34.1 & 34.5 & 34.7 & 35.1 & 35.4 & 35.7 & 36.0 & 36.4 & 36.7 & 37.0 & 37.3 & 37.7 & 38.0 \\
\hline 14.0 & . & & & & & & & & 35.7 & 36.0 & 36.3 & 36.7 & 37.0 & 37.3 & 37.7 & 38.0 & 38.3 & 38.7 & 39.0 & 39.4 \\
\hline 14.5 & & & & & & & & & 36.9 & 37.2 & 37.5 & 37.9 & 38.3 & 38.6 & 39.0 & 39.3 & 39.7 & 40.0 & 40.4 & 40.7 \\
\hline 15.0 & & & & & & & & & 38.1 & 38.5 & 38.8 & 39.2 & 39.5 & 39.9 & 40.3 & 40.6 & 41.0 & 41.3 & 41.7 & 42.1 \\
\hline 15.5 & & & & & & & & & 39.3 & 39.7 & 40.1 & 40.4 & 40.8 & 41.2 & 41.6 & 41.9 & 42.3 & 42.7 & 43.0 & 43.4 \\
\hline 16.0 & & & & & & & & & & 40.9 & 41.3 & 41.7 & 42.1 & 42.5 & 42.9 & 43.2 & 43.6 & 44.0 & 44.4 & 44.8 \\
\hline 16.5 & & & & & & & & & & 42.2 & 42.6 & 43.0 & 43.4 & 43.8 & 44.2 & 44.6 & 45.0 & 45.3 & 45.7 & 46.2 \\
\hline 17.0 & & & & & & & & & & 43.4 & 43.8 & 44.2 & 44.6 & 45.0 & 45.5 & 45.9 & 46.3 & 46.7 & 47.1 & 47.5 \\
\hline 17.5 & & & & & & & & & & 44.7 & 45.1 & 45.5 & 45.9 & 46.3 & 46.8 & 47.2 & 47.6 & 48.0 & 48.5 & 48. \\
\hline 18.0 & & & & & & & & & & 45.9 & 46.3 & 46.8 & 47.2 & 47.6 & 48.1 & 48.5 & 48.9 & 49.4 & 49.8 & 50. \\
\hline 18.5 & & & & & & & & & & & 47.6 & 48.0 & 48.5 & 48.9 & 49.4 & 49.8 & 50.3 & 50.7 & 51.1 & 51. \\
\hline 19.0 & & & & & & & & & & & 48.9 & 49.3 & 49.8 & 50.2 & 50.7 & 51.1 & 51.6 & 52.1 & 52.5 & 53. \\
\hline 19.5 & & & & & & & & & & & 50.1 & 50.6 & 51.0 & 51.5 & 52.0 & $\mid 52.4$ & 52.9 & $\mid 53.4$ & $\mid 53.9$ & 54 \\
\hline
\end{tabular}


TABLE C (concluded)

\begin{tabular}{|c|c|c|c|c|c|c|c|c|c|c|c|c|c|c|c|c|c|c|c|c|}
\hline$\Rightarrow \theta_{d}$ & $=311$ & 313 & 315 & 317 & 319 & 321 & 323 & 325 & 327 & 329 & 331 & 333 & 335 & 337 & 339 & 341 & 343 & 345 & 347 & 349 \\
\hline 5.0 & 14.9 & 15.0 & 15.1 & 15.3 & 15.4 & 15.5 & 15.6 & 15.7 & 15.9 & 16.0 & 16.1 & 16.2 & 16.4 & 16.5 & 16.6 & 16.7 & 16.9 & 17.0 & 17.1 & 17.2 \\
\hline 5.5 & 16.3 & 16.4 & 16.5 & 16.7 & 16.8 & 17.0 & 17.1 & 17.2 & 17.4 & 17.5 & 17.6 & 17.8 & 17.9 & 18.1 & 18.2 & 18.3 & 18.4 & 18.6 & 18.7 & 18.9 \\
\hline 6.0 & 17.7 & 17.8 & 18.0 & 18.1 & 18.3 & 18.4 & 18.6 & 18.7 & 18.8 & 19.0 & 19.1 & 19.3 & 19.4 & 19.6 & 19.7 & 19.9 & 20.0 & 20.2 & 20.3 & 20.5 \\
\hline 6.5 & 19.1 & 19.2 & 19.4 & 19.5 & 19.7 & 19.9 & 20.0 & 20.2 & 20.3 & 20.5 & 20.6 & 20.8 & 21.0 & 21.1 & 21.3 & 21.4 & 21.6 & 21.8 & 21.9 & 22.1 \\
\hline 7.0 & 20.5 & 20.6 & 20.8 & 21.0 & 21.1 & 21.3 & 21.5 & 21.7 & 21.8 & 22.0 & 22.2 & 22.3 & 22.5 & 22.7 & 22.8 & 23.0 & 23.2 & 23.4 & 23.6 & 23.7 \\
\hline 7.5 & 21.8 & 22.0 & 22.2 & 22.4 & 22.6 & 22.8 & 22.9 & 23.1 & 23.3 & 23.5 & 23.7 & 23.9 & 24.0 & 24.2 & 24.4 & 24.6 & 24.8 & 25.0 & 25.2 & 25.3 \\
\hline 8.0 & 23.2 & 23.4 & 23.6 & 23.8 & 24.0 & 24.2 & 24.4 & 24.6 & 24.8 & 25.0 & 25.2 & 25.4 & 25.6 & 25.7 & 25.9 & 26.1 & 26.3 & 26.5 & 26.7 & 26.9 \\
\hline 8.5 & 24.6 & 24.8 & 25.0 & 25.2 & 25.4 & 25.6 & 25.8 & 26.0 & 26.2 & 26.4 & 26.7 & 26.9 & 27.1 & 27.3 & 27.5 & 27.7 & 27.9 & 28.1 & $28.3^{4}$ & 28.5 \\
\hline 9.0 & 26.0 & 26.2 & 26.4 & 26.6 & 26.8 & 27.0 & 27.3 & 27.5 & 27.7 & 27.9 & 28.2 & 28.4 & 28.6 & 28.8 & 29.0 & 29.3 & 29.5 & 29.7 & 29.9 & 30.2 \\
\hline 9.5 & 27.4 & 27.6 & 27.8 & 28.0 & 28.3 & 28.5 & 28.7 & 29.0 & 29.2 & 29.4 & 29.7 & 29.9 & 30.1 & 30.4 & 30.6 & 30.8 & 31.1 & 31.3 & 31.5 & 31.8 \\
\hline 10.0 & 28.7 & 29.0 & 29.2 & 29.4 & 29.7 & 29.9 & 30.2 & 30.4 & 30.6 & 30.9 & 31.2 & 31.4 & 31.6 & 31.9 & 32.1 & & & & & \\
\hline 10.5 & 30.1 & 30.3 & 30.6 & 30.9 & 31.1 & 31.4 & 31.6 & 31.9 & 32.1 & 32.4 & 32.7 & 32.9 & 33.2 & 33.4 & 33.7 & & & & & \\
\hline 11.0 & 31.5 & 31.7 & 32.0 & 32.3 & 32.5 & 32.8 & 33.1 & 33.4 & 33.6 & 33.9 & 34.2 & 34.4 & 34.7 & 35.0 & 35.2 & & & & & \\
\hline 11.5 & 32.8 & 33.1 & 33.4 & 33.7 & 33.9 & 34.2 & 34.5 & 34.8 & 35.1 & 35.4 & 35.7 & 35.9 & 36.2 & 36.5 & 36.8 & & & & & \\
\hline 12.0 & 34.2 & 34.5 & 34.8 & 35.1 & 35.4 & 35.6 & 35.9 & 36.2 & 36.5 & 36.8 & 37.1 & 37.4 & 37.7 & 38.0 & 38.3 & & & & & \\
\hline 12.5 & 35.6 & 35.9 & 36.2 & 36.5 & 36.8 & 37.1 & 37.4 & 37.7 & 38.0 & 38.3 & 38.6 & 38.9 & 39.2 & 39.5 & 39.9 & & & & & \\
\hline 13.0 & 37.0 & 37.3 & 37.6 & 37.9 & 38.2 & 38.5 & 38.9 & 39.2 & 39.5 & 39.8 & 40.1 & 40.4 & 40.8 & 41.1 & 41.4 & & & & & \\
\hline 13.5 & 38.3 & 38.6 & 39.0 & 39.3 & 39.6 & 40.0 & 40.3 & 40.6 & 40.8 & 41.3 & 41.6 & 41.9 & 42.3 & 42.6 & 42.9 & & & & & \\
\hline 4.0 & 39.7 & 40.0 & 40.4 & 40.7 & 41.0 & 41.4 & 41.7 & 42.1 & 42.4 & 42.8 & 43.1 & 43.4 & 43.8 & 44.1 & 44.5 & & & & & \\
\hline 14.5 & 41.1 & 41.4 & 41.8 & 42.1 & 42.5 & 42.8 & 43.2 & 43.5 & 43.9 & 44.3 & 44.6 & 45.0 & 45.3 & 45.7 & 46.0 & & & & & \\
\hline 15.0 & 42.4 & 42.8 & 43.2 & 43.5 & 43.9 & 44.3 & 44.6 & 45.0 & 45.4 & 45.7 & 46.1 & 46.5 & 46.8 & 47.2 & 47.6 & & & & & \\
\hline 15.5 & 43.8 & 44.2 & 44.6 & 44.9 & 45.3 & 45.7 & 46.1 & 46.5 & 46.8 & 47.2 & 47.6 & 48.0 & 48.3 & 48.7 & 49.1 & & & & & \\
\hline 16.0 & 45.2 & 45.6 & 46.0 & 46.3 & 46.7 & 47.1 & 47.5 & 47.9 & 48.3 & 48.7 & 49.1 & 49.5 & 49.9 & 50.3 & 50.7 & & & & & \\
\hline 16.5 & 46.6 & 47.0 & 47.3 & 47.7 & 48.2 & 48.6 & 49.0 & 49.4 & 49.8 & 50.2 & 50.6 & 51.0 & 51.4 & 51.8 & 52.2 & & & & & \\
\hline 17.0 & 47.9 & 48.3 & 48.8 & 49.2 & 49.6 & 50.0 & 50.4 & 50.8 & 51.3 & 51.7 & 52.1 & 52.5 & 52.9 & 53.3 & 53.8 & & & & & \\
\hline 17.5 & 49.3 & 49.7 & 50.1 & 50.6 & 51.0 & 51.4 & 51.9 & 52.3 & 52.7 & 53.1 & 53.6 & 54.0 & 54.4 & 54.9 & 55.3 & & & & & \\
\hline 18.0 & 50.7 & 51.1 & 51.6 & 52.0 & 52.4 & 52.9 & 53.3 & 53.8 & 54.2 & 54.6 & 55.1 & 55.5 & 56.0 & 56.4 & 56.9 & & & & & \\
\hline 18.5 & 52.1 & 52.5 & 53.0 & 53.4 & 53.9 & 54.3 & 54.8 & 55.2 & 55.7 & 56.1 & 56.6 & 57.0 & 57.5 & 58.0 & 58.4 & & & & & \\
\hline 19.0 & 53.5 & 53.9 & 54.4 & 54.8 & 55.3 & 55.8 & 56.2 & 56.7 & 57.2 & 57.6 & 58.1 & 58.6 & 59.1 & 59.5 & 60.0 & & & & & \\
\hline 19.5 & $|54.8|$ & 55.3 & 55.8 & 56.2 & 56.7 & 57.2 & 57.7 & 58.1 & 58.6 & 59.1 & 59.6 & 60.1 & 60.6 & 61.1 & 61.5 & & & & & \\
\hline
\end{tabular}




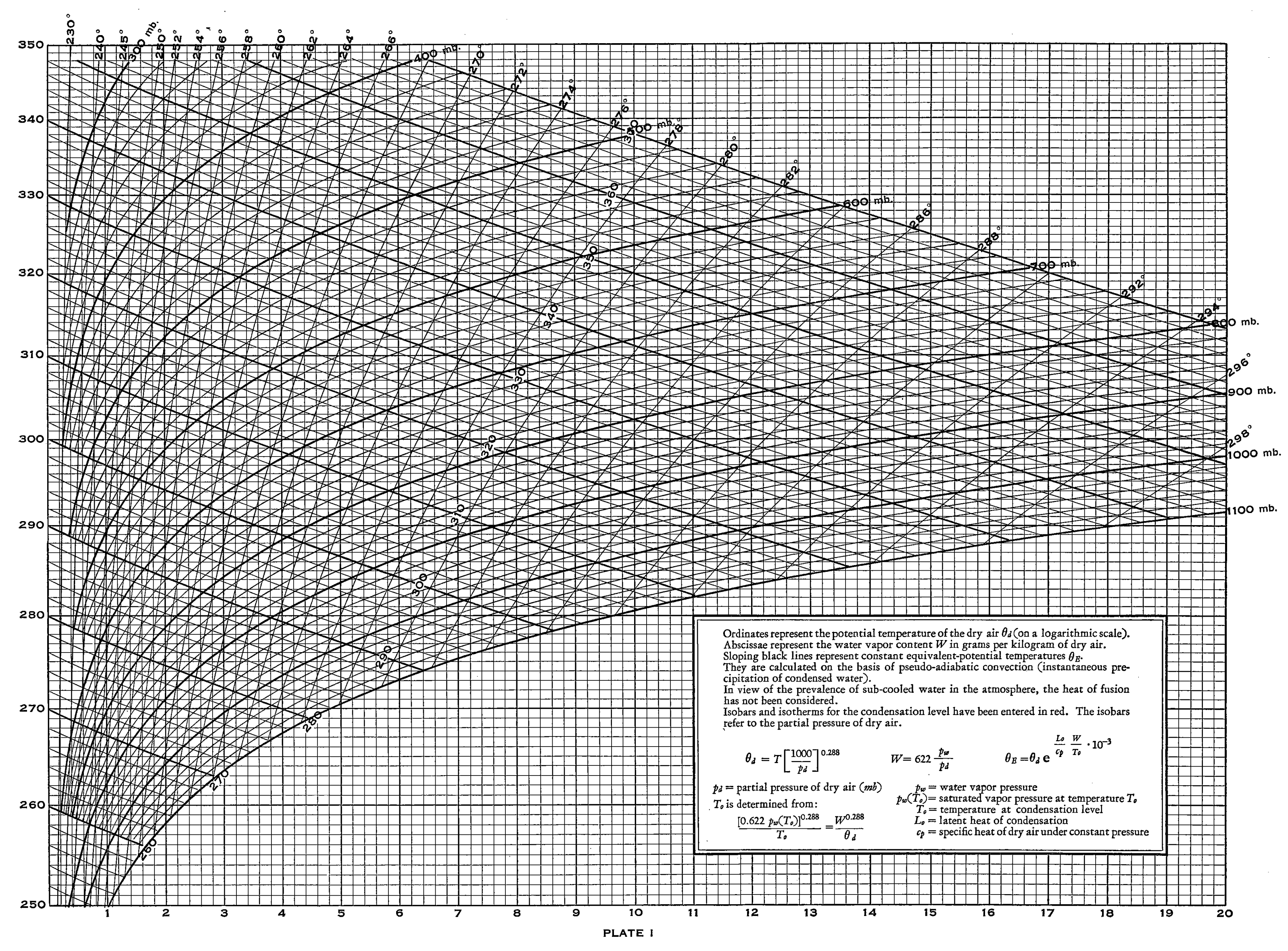




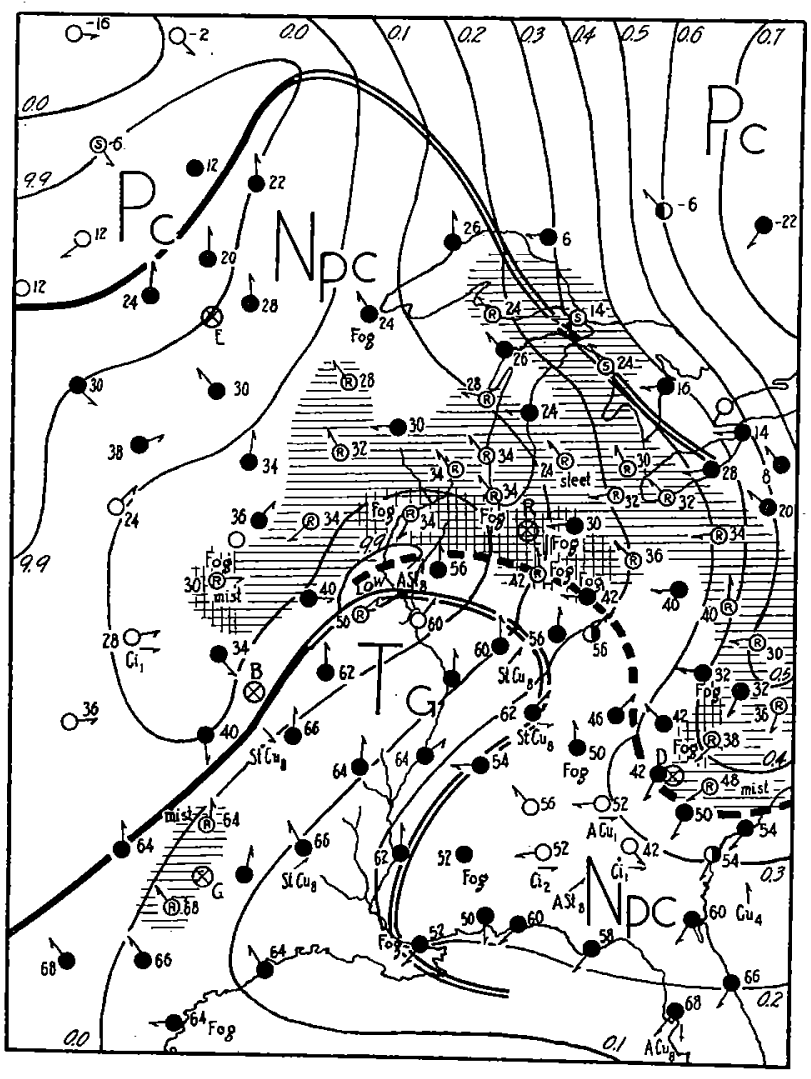

DECEMBER $12,1929,8$ A. M.

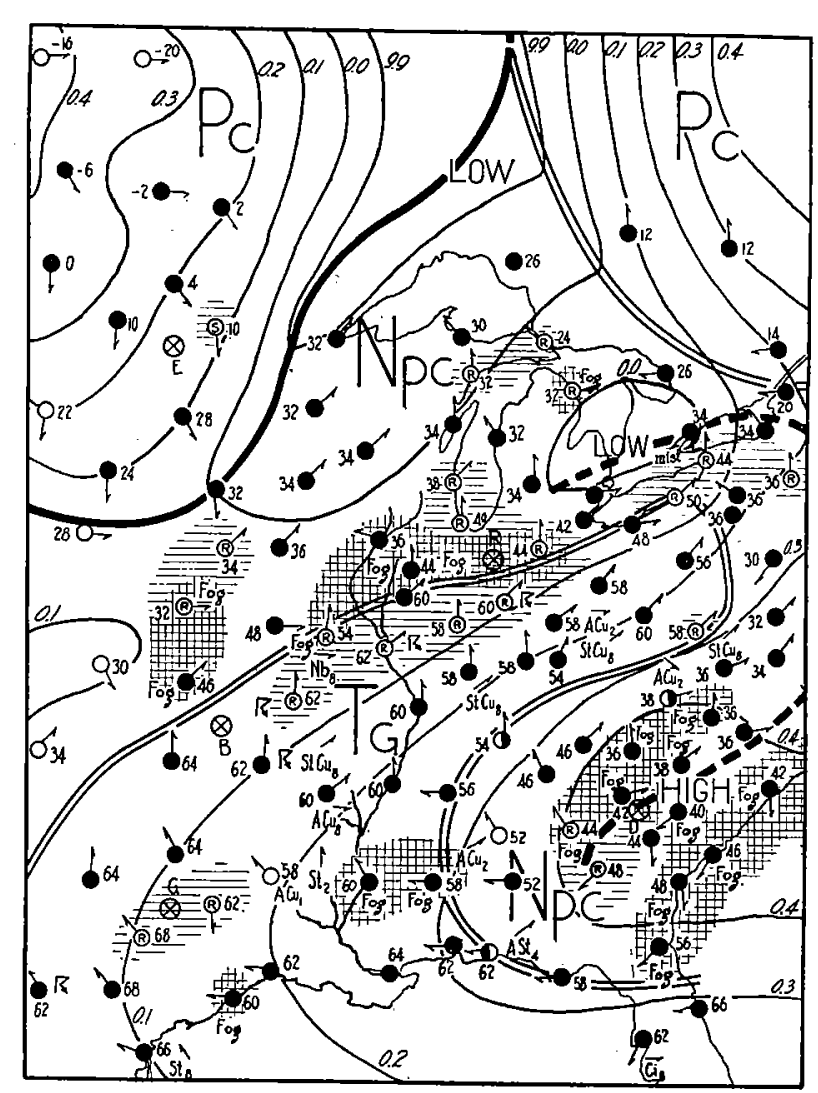

DECEMBERं 13, 1929, 8 A. M.

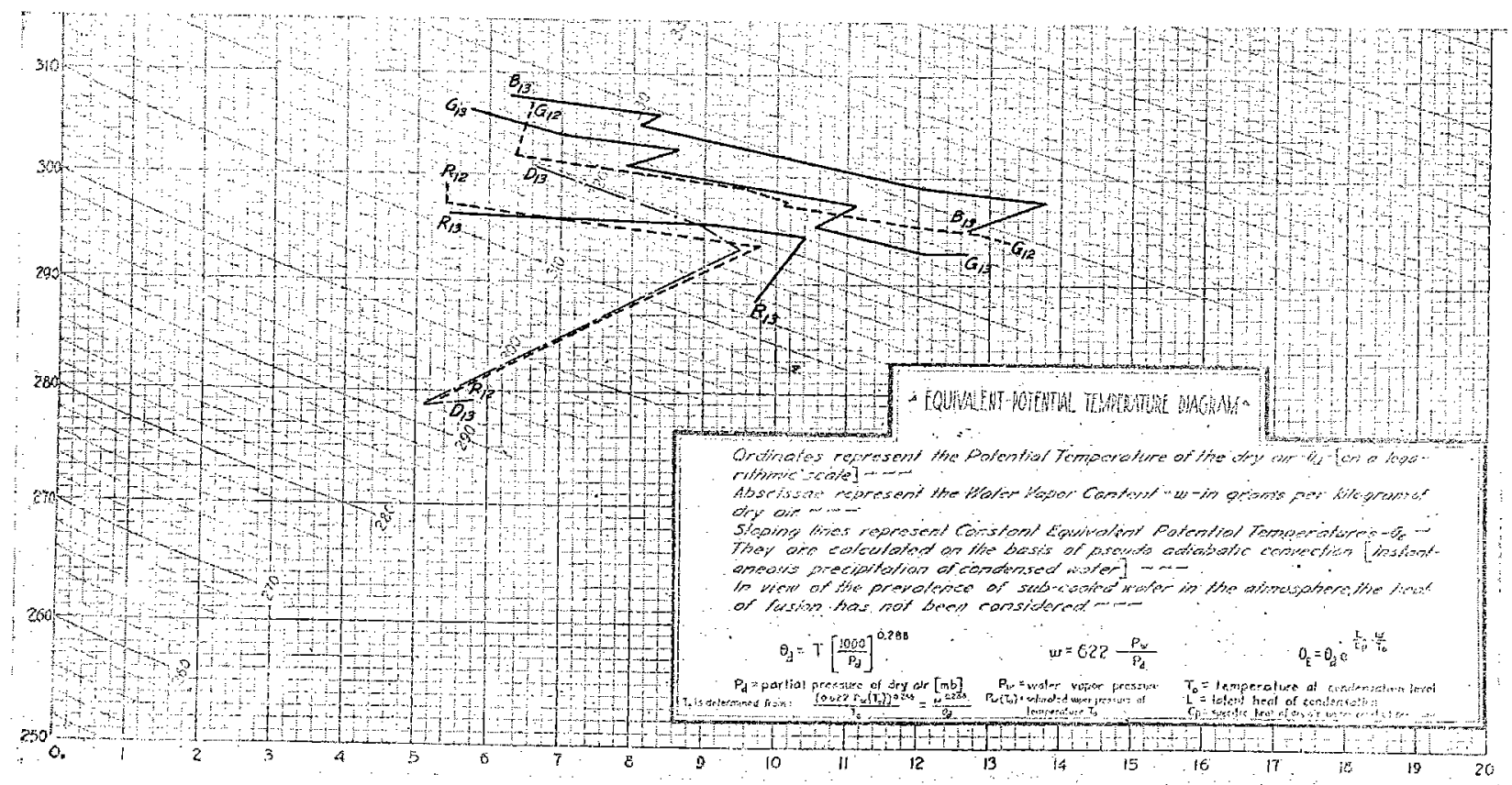

PLATE I] 


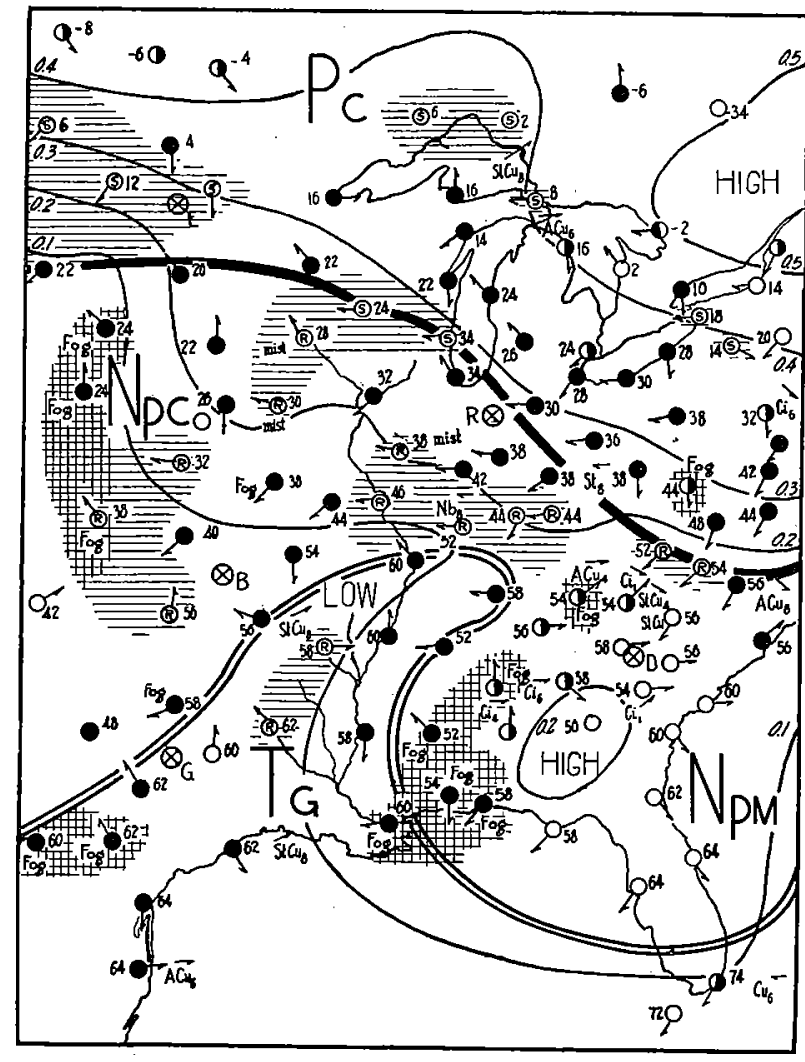

DECEMBER 1.6, 1929, 8 A. M.

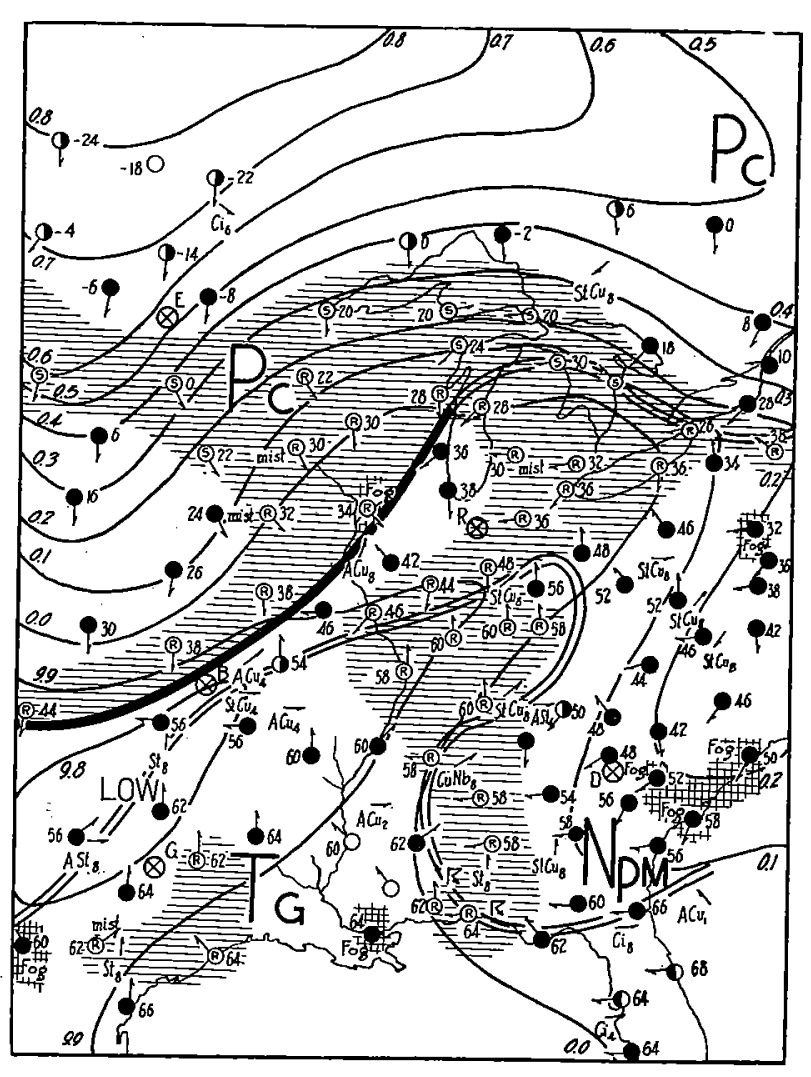

DECEMBER 17, 1929, 8 A. M.

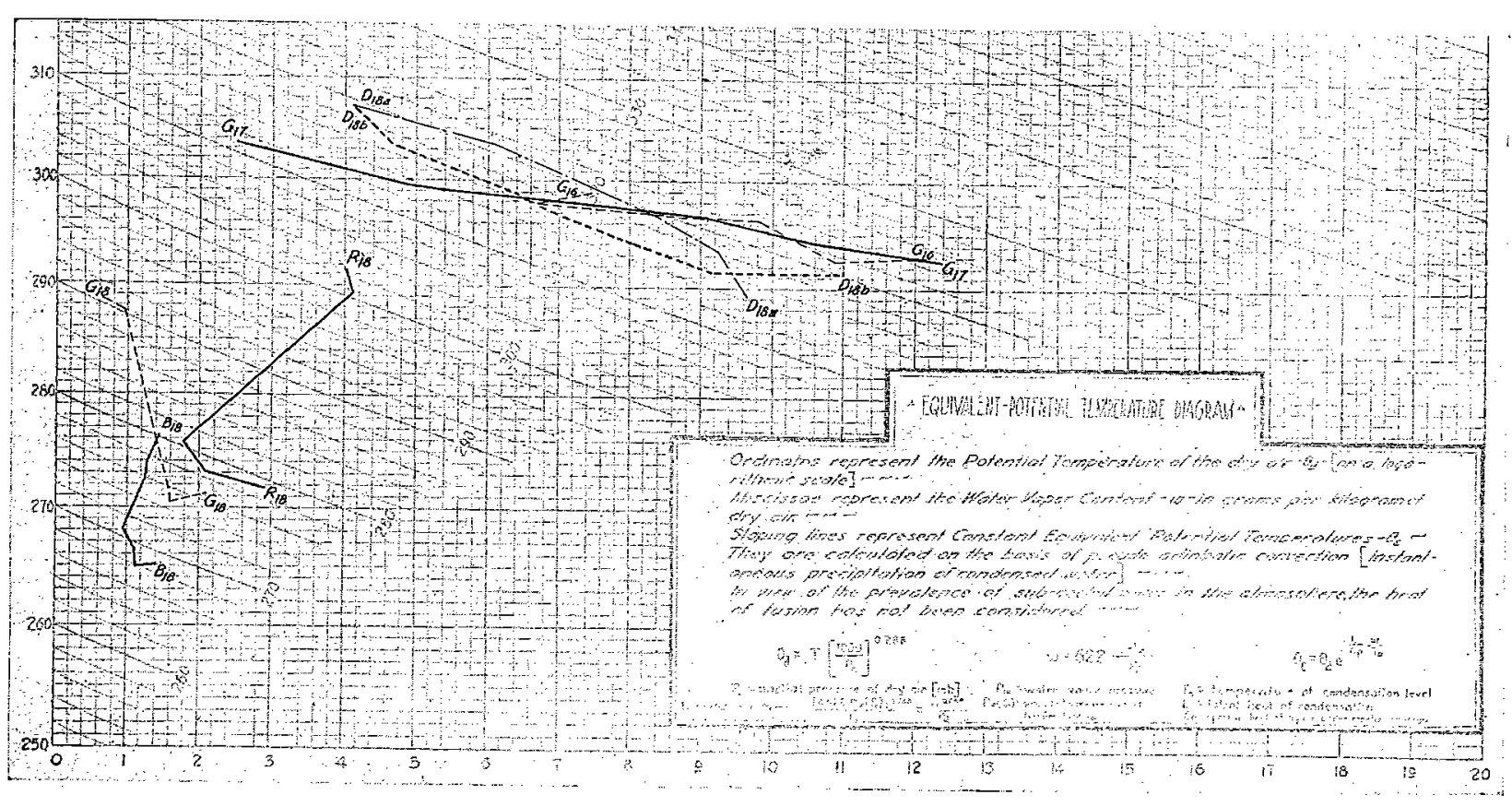

PLATE III 


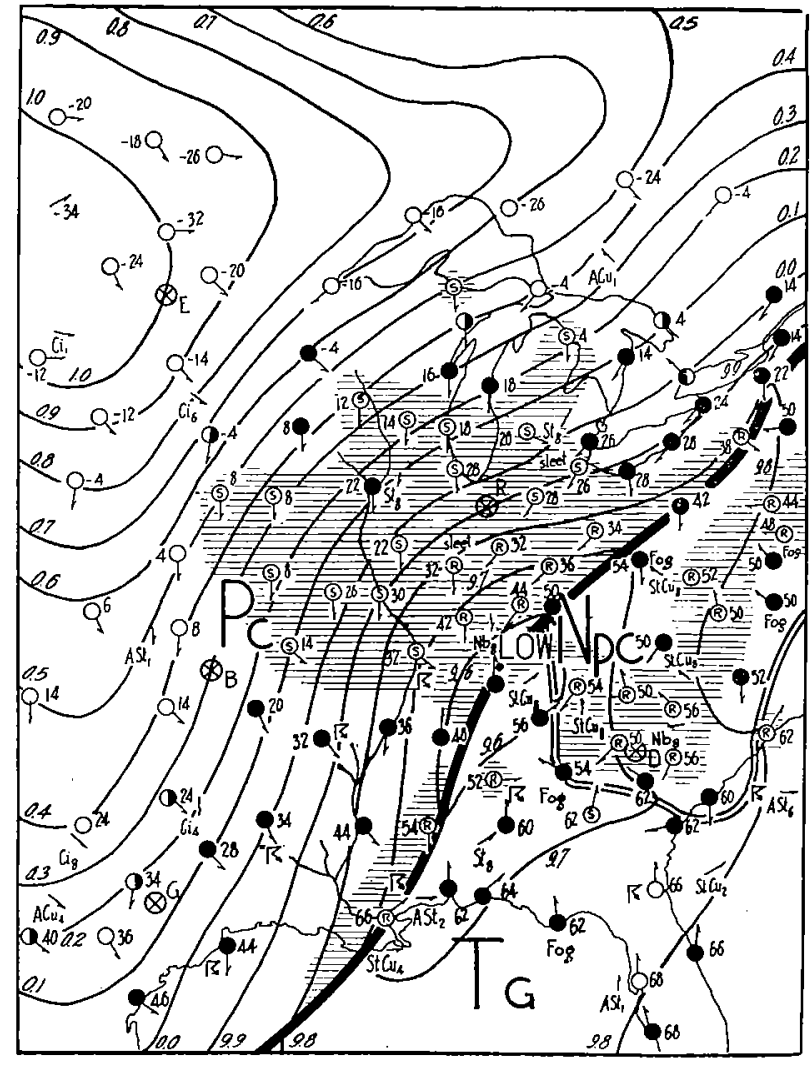

DECEMBER $18,1929,8$ A. M.

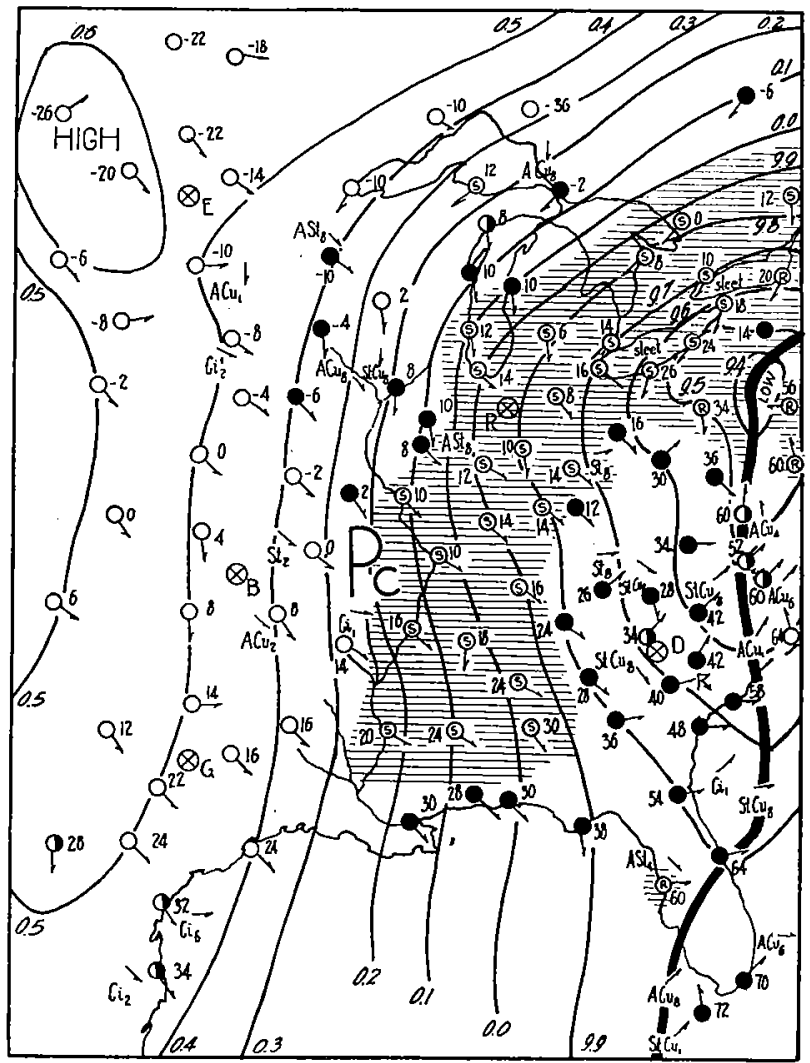

DECEMBER 19, 1929, 8 A. M.

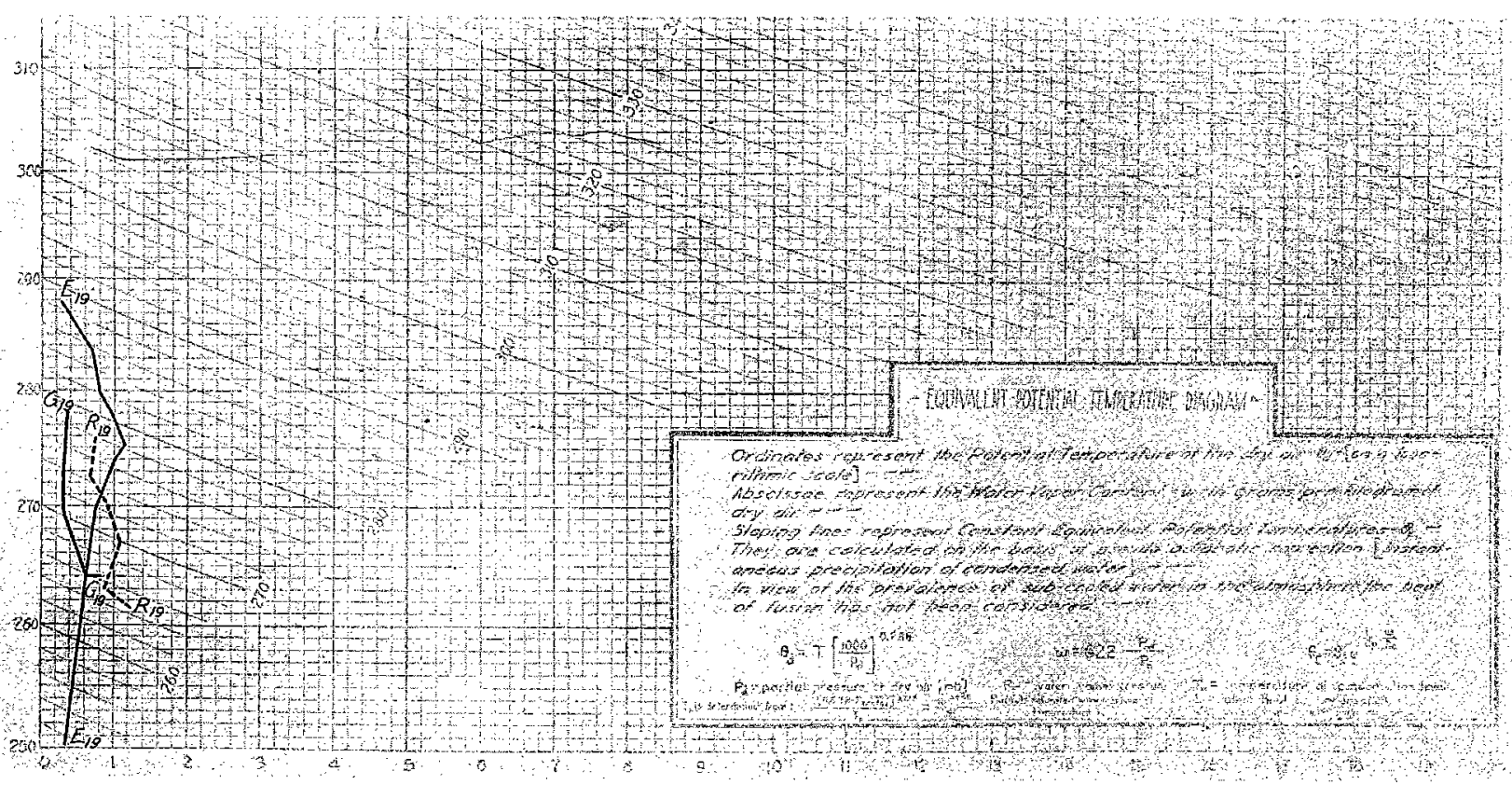

PLATE IV 

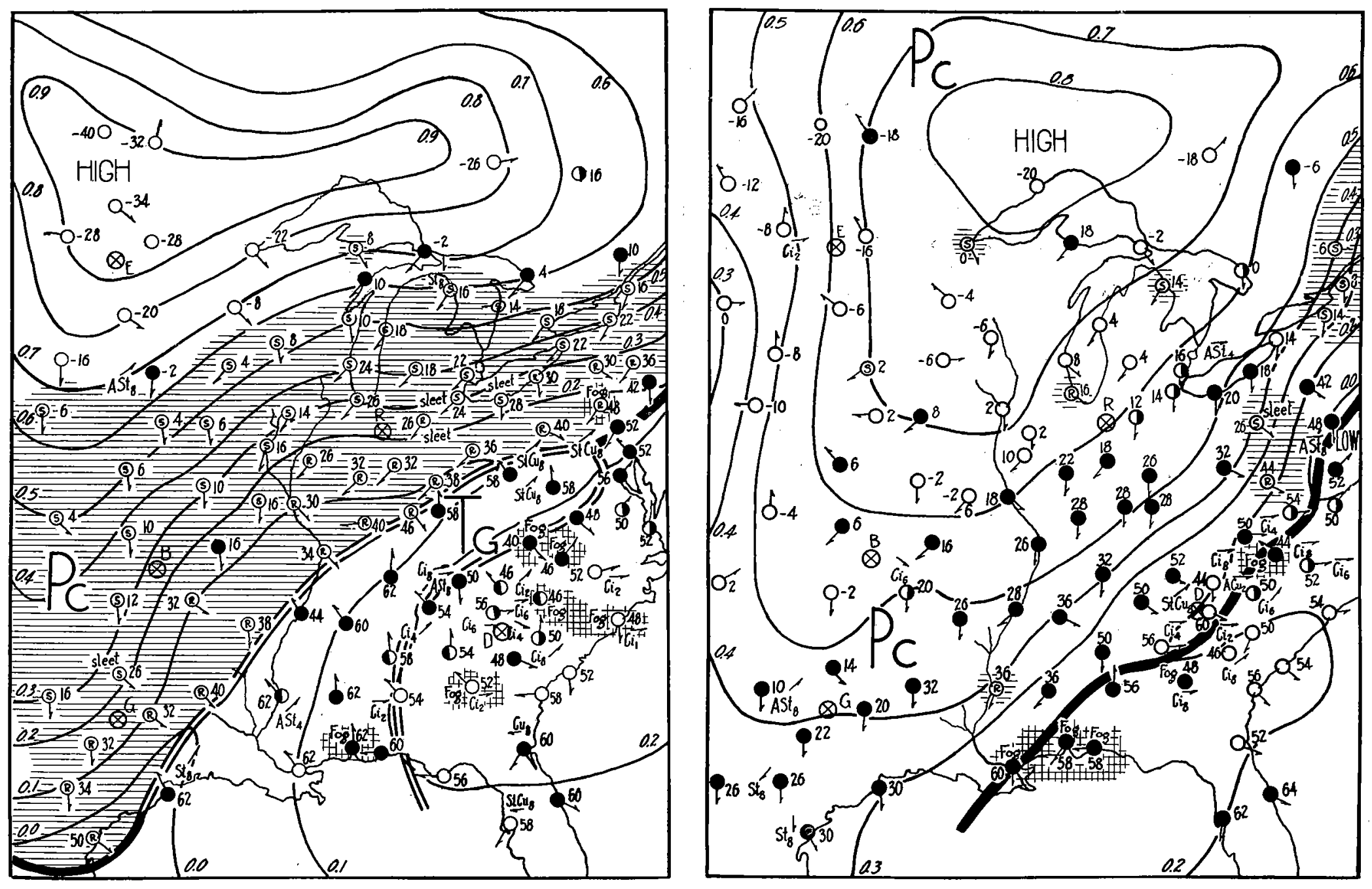

JANUARY 9, 1930, 8 A. M.

PLATE V

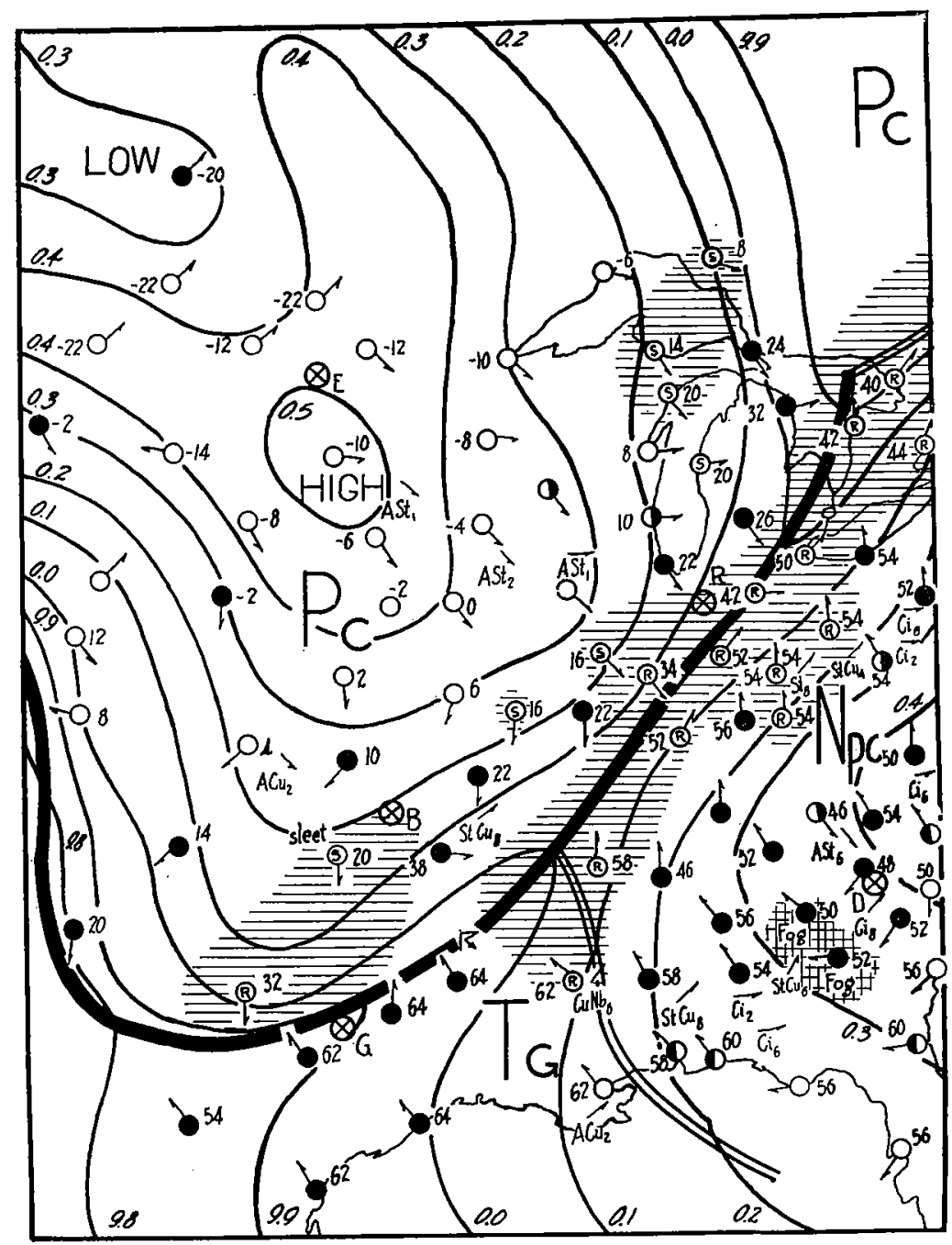

JANUARY 7, 1930, 8 A. M.

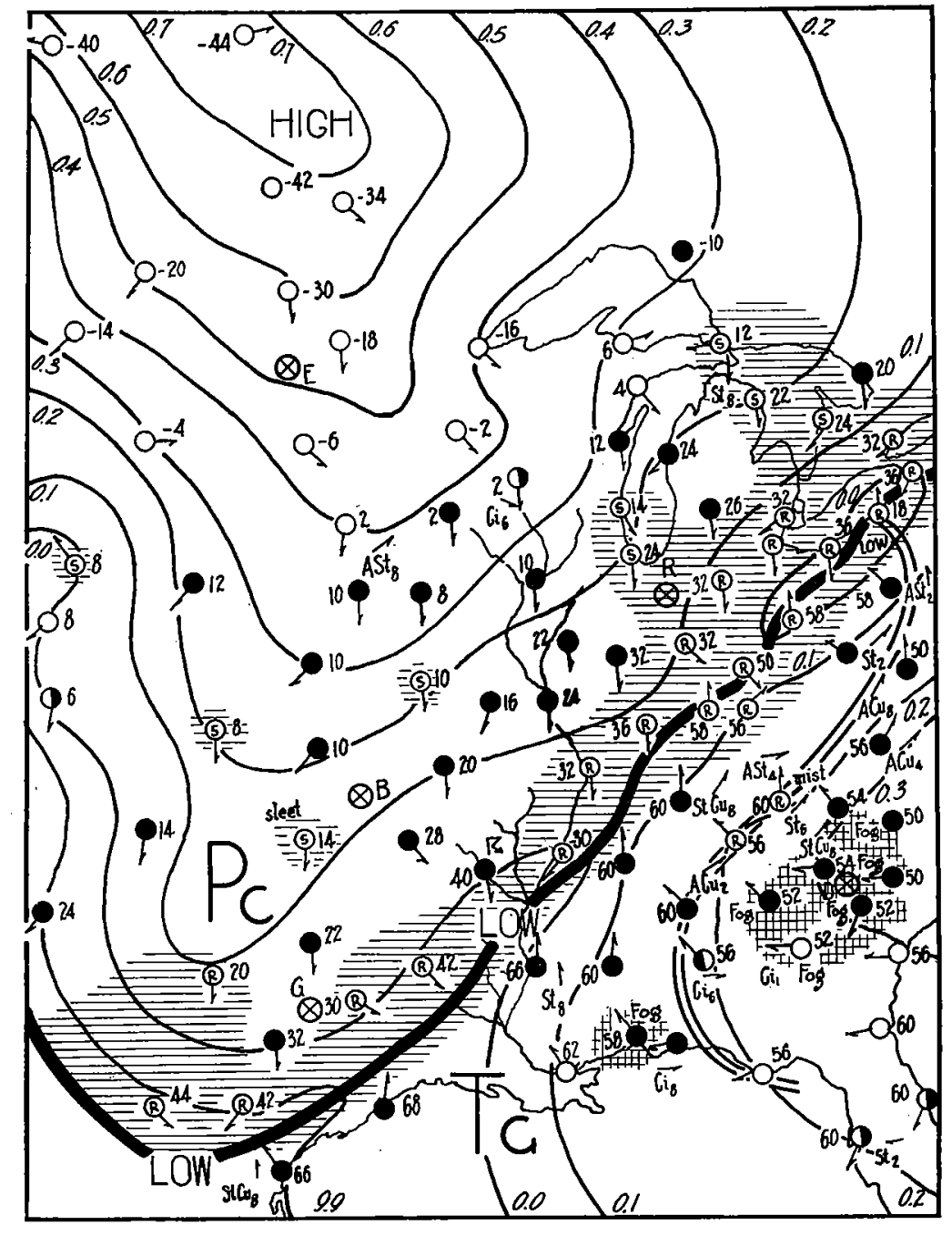

JANUARY $8,1930,8$ A.M. 


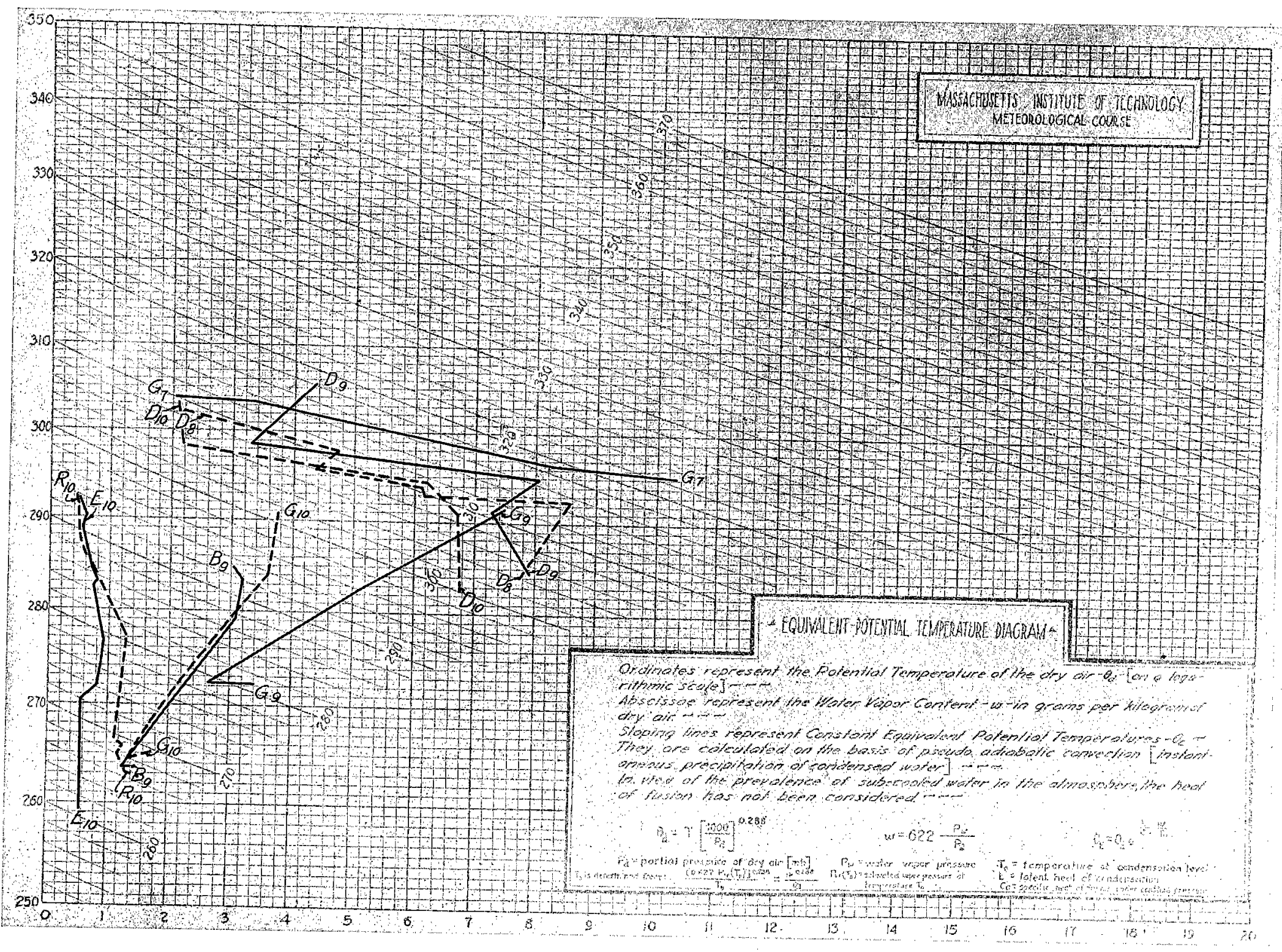

CHARACTERISTIC CURVES, JANUARY 7-10, 1930 


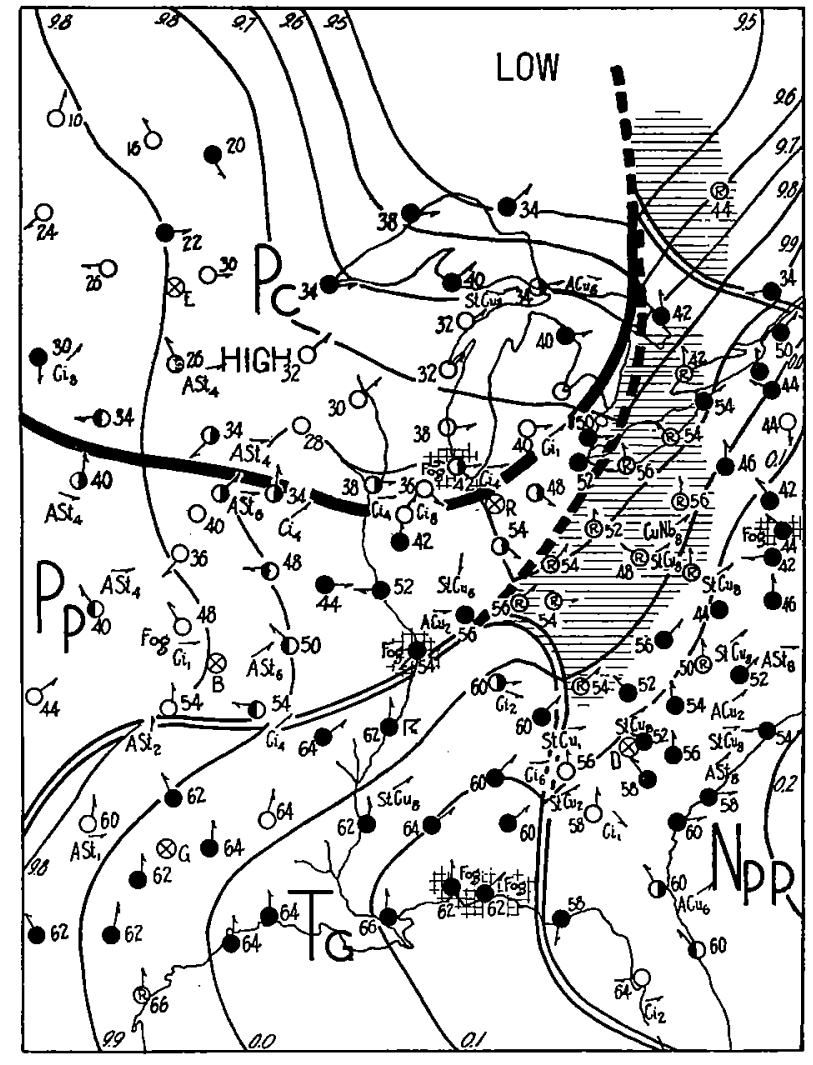

FEBRUARY $23,1930,8$ A. M.

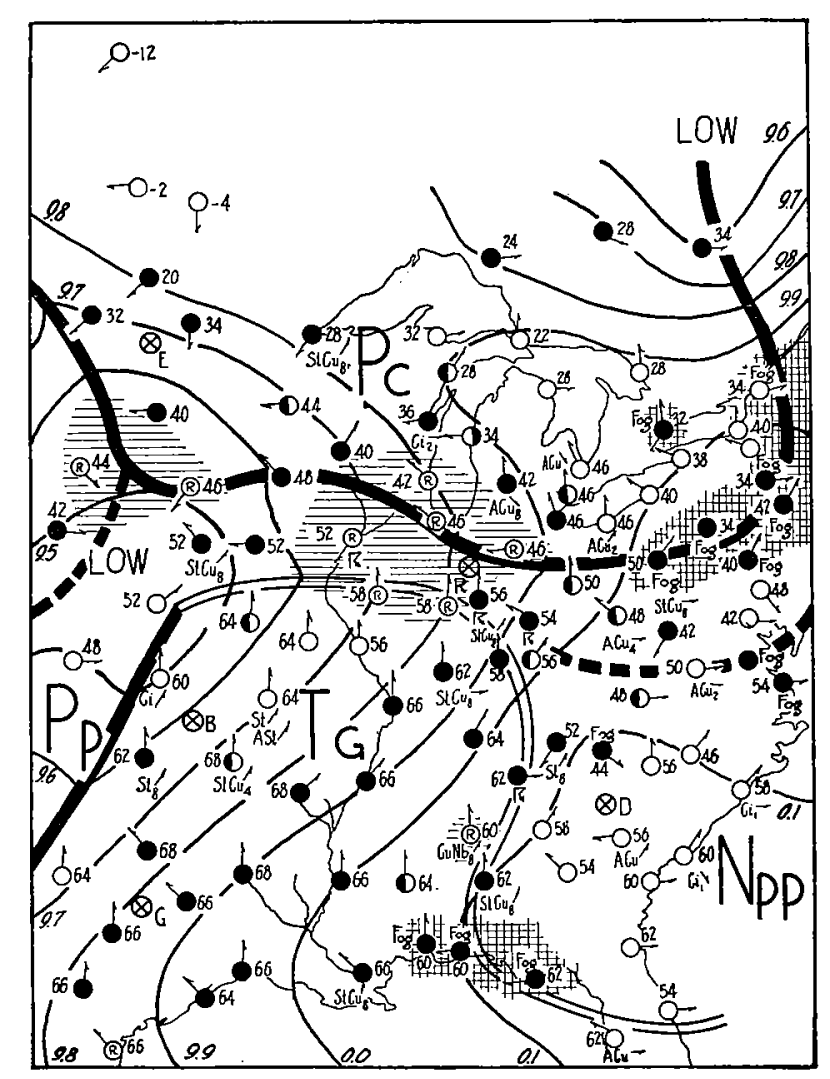

FEBRUARY 24, 1930; 8 A. M.

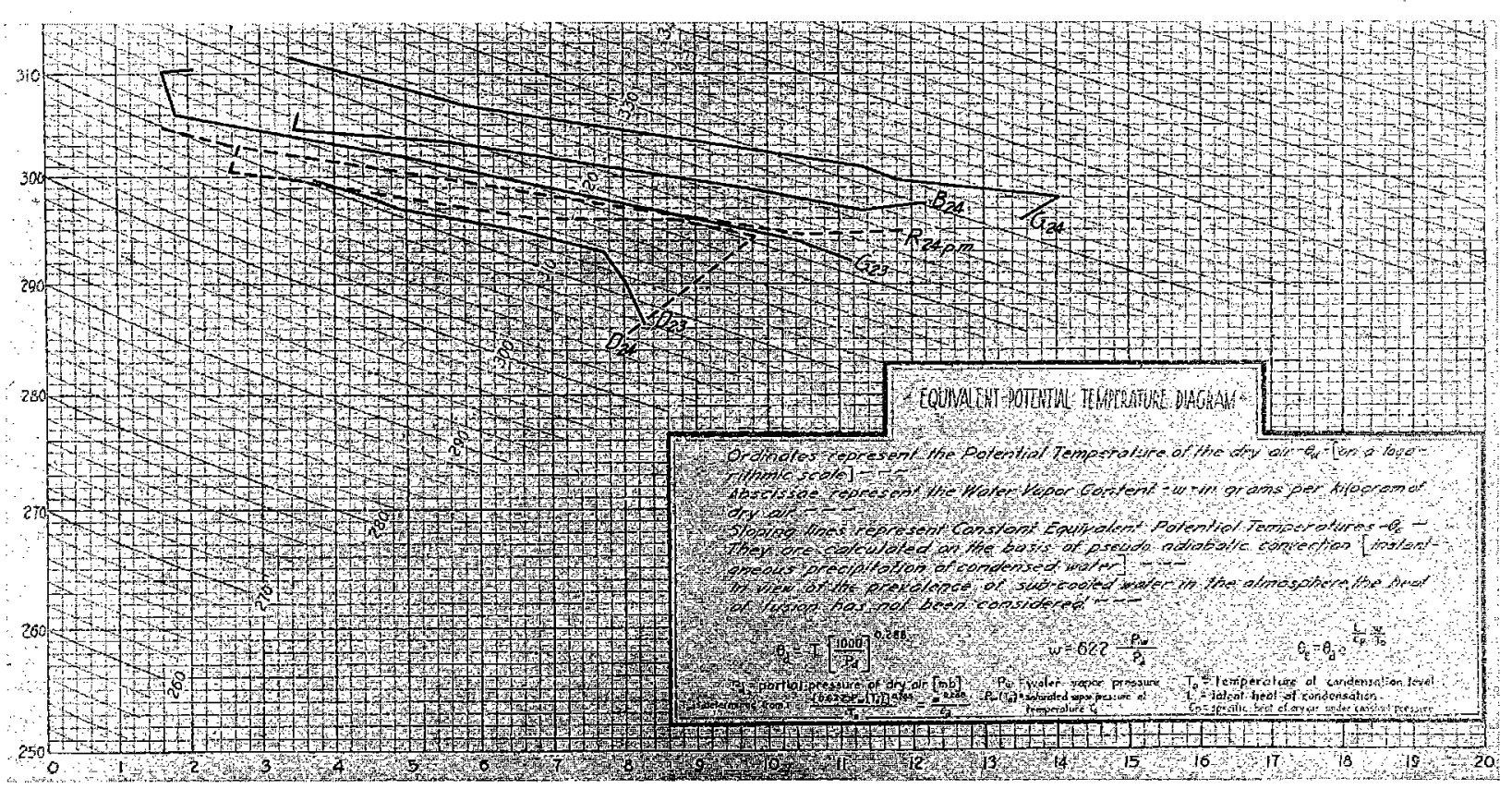




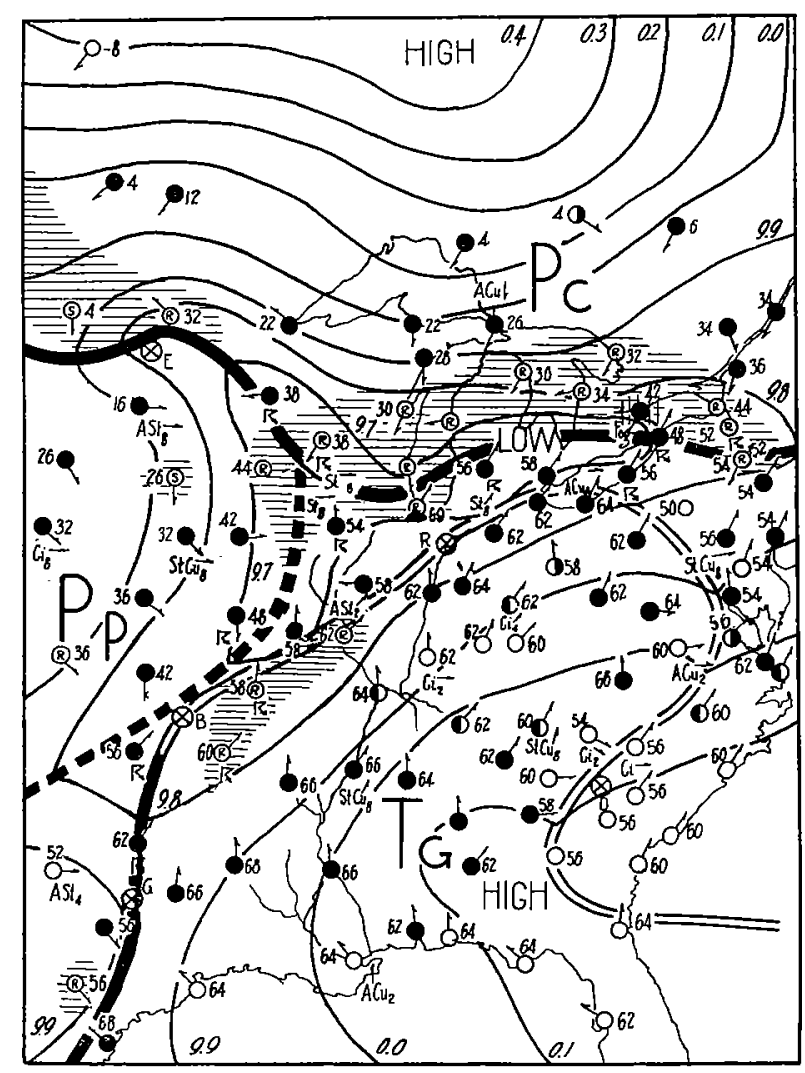

FEBRUARY 25, 1930, 8 A. M.

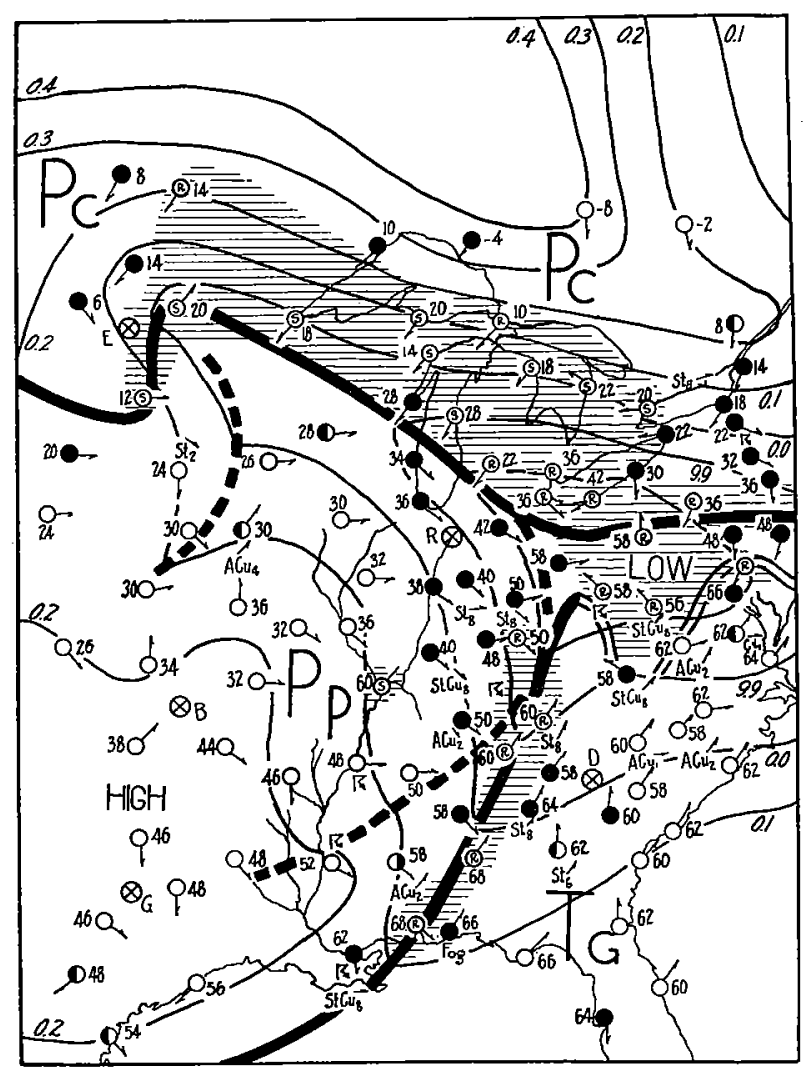

FEBRUARY 26; 1930, 8 A. M.

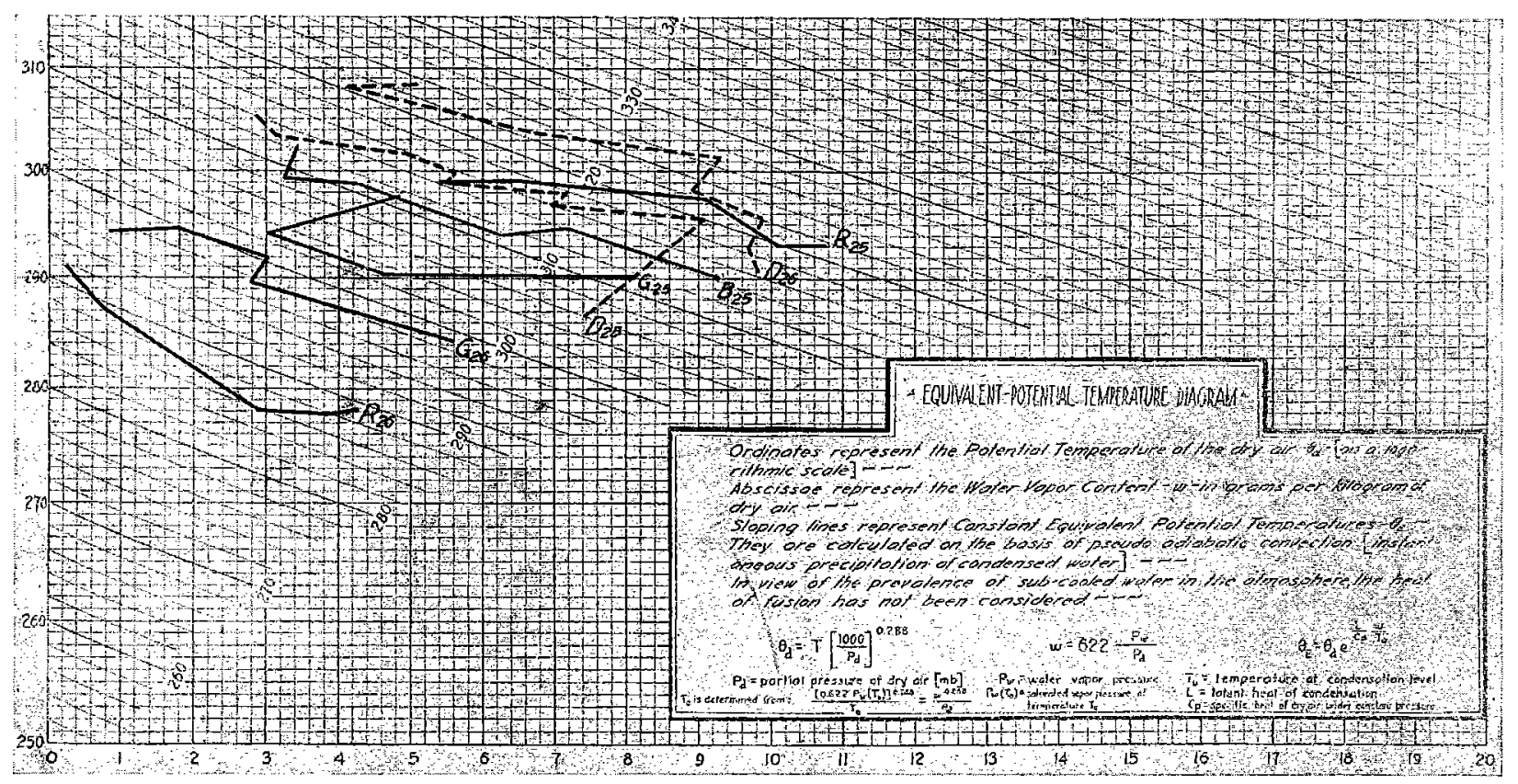

PLATE VIII 

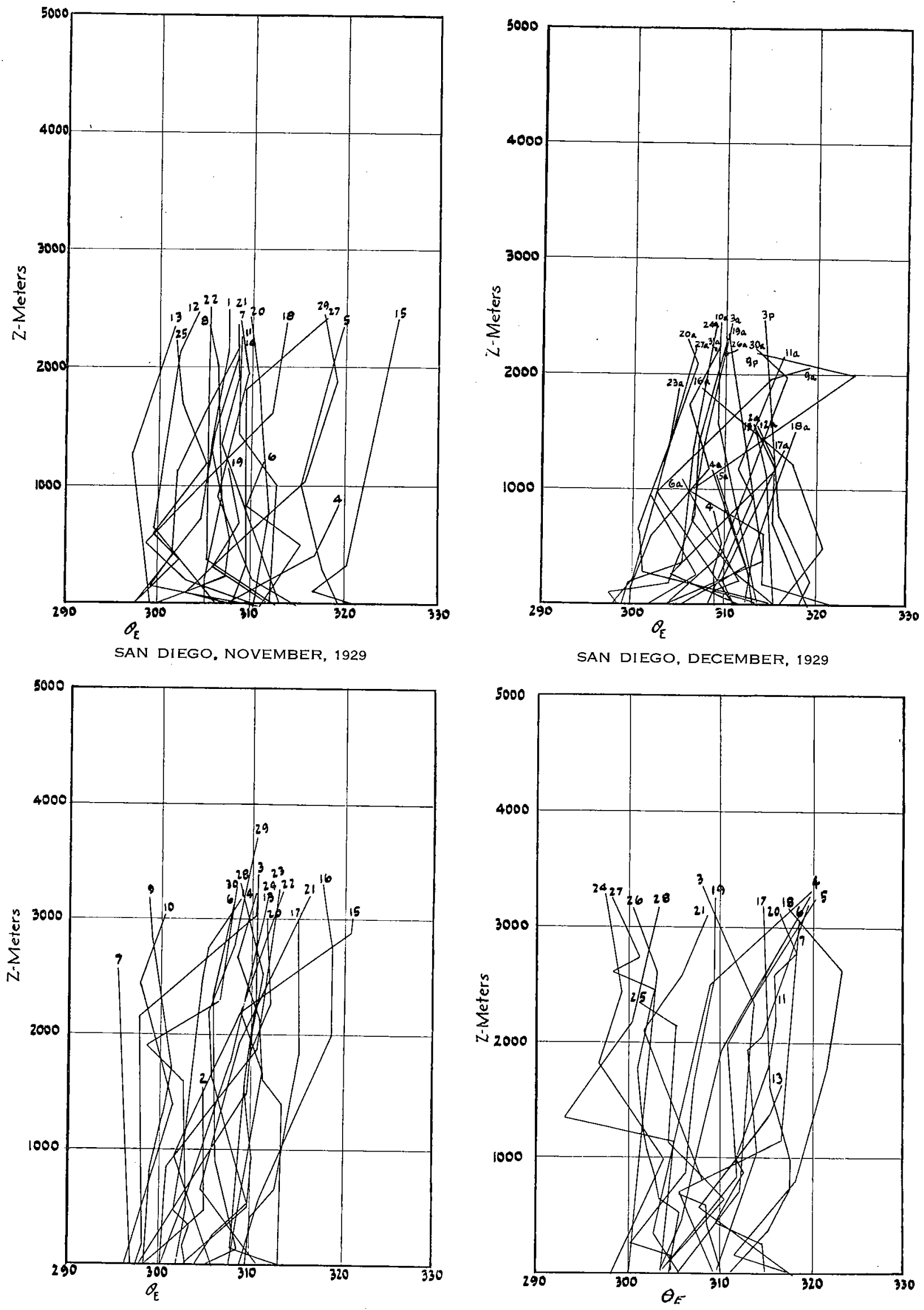

SAN DIEGO, JANUARY, 1930

PLATE IX

SAN DIEGO, FEBRUARY, 1930 

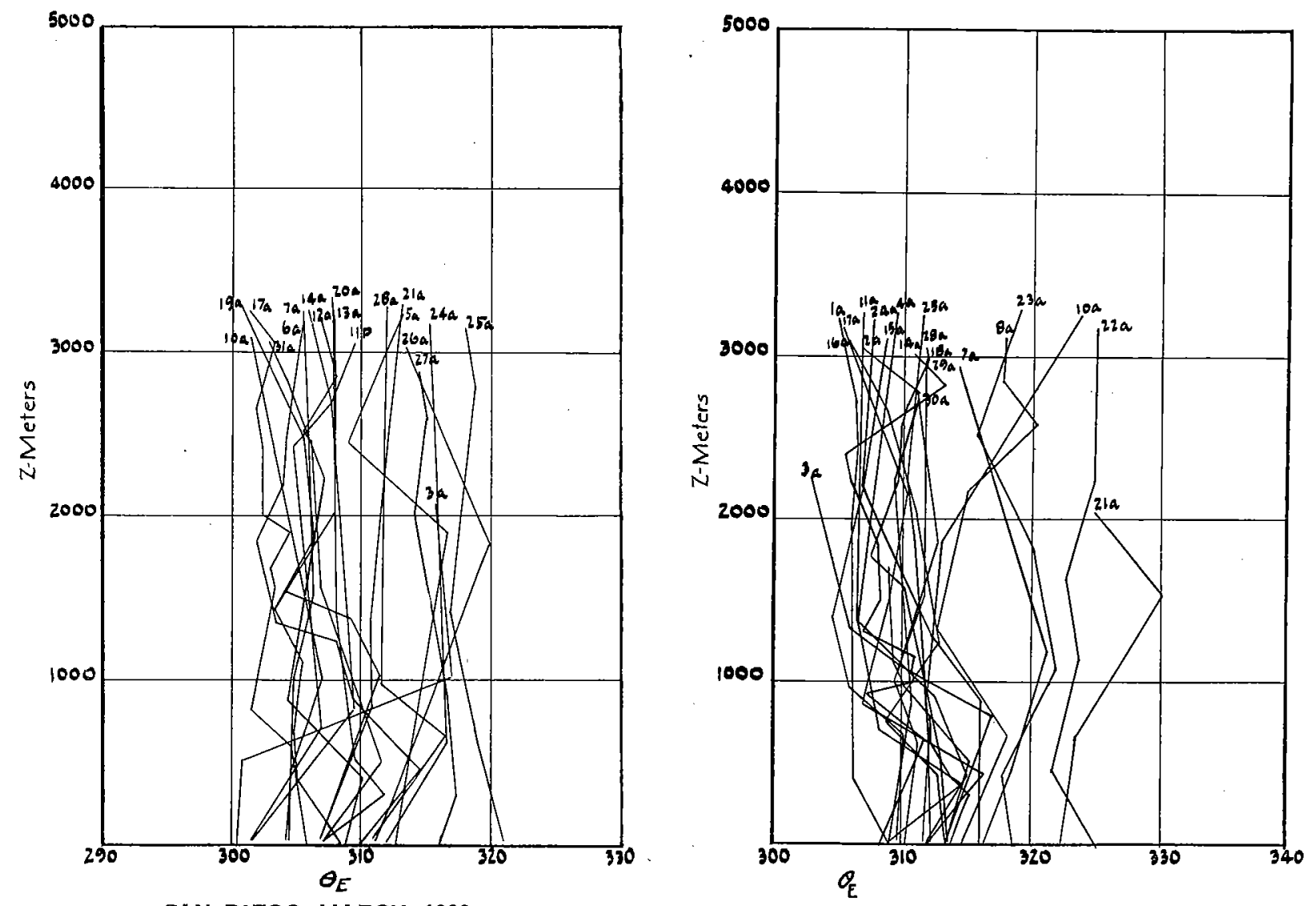

SAN DIEGO, MARCH, 1930
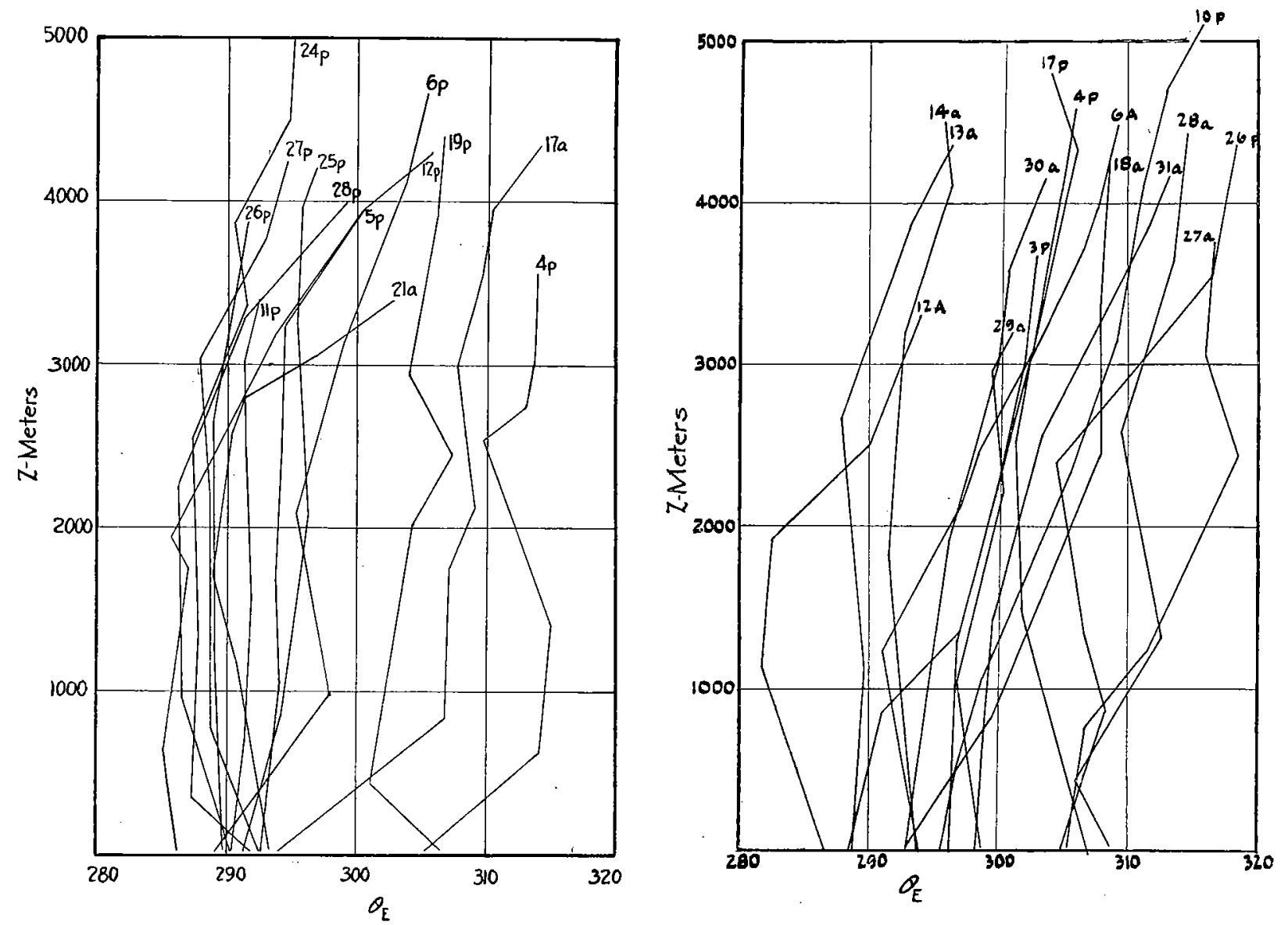

SEATTLE, FEBRUARY, 1930

PLATE $X$ 


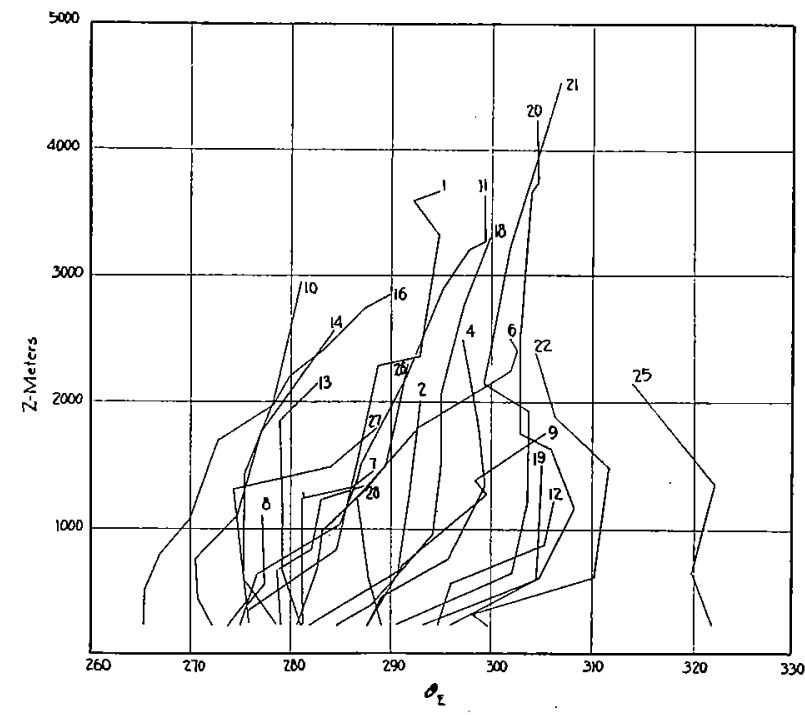

ROYAL CENTER, FEBRUARY, 1930

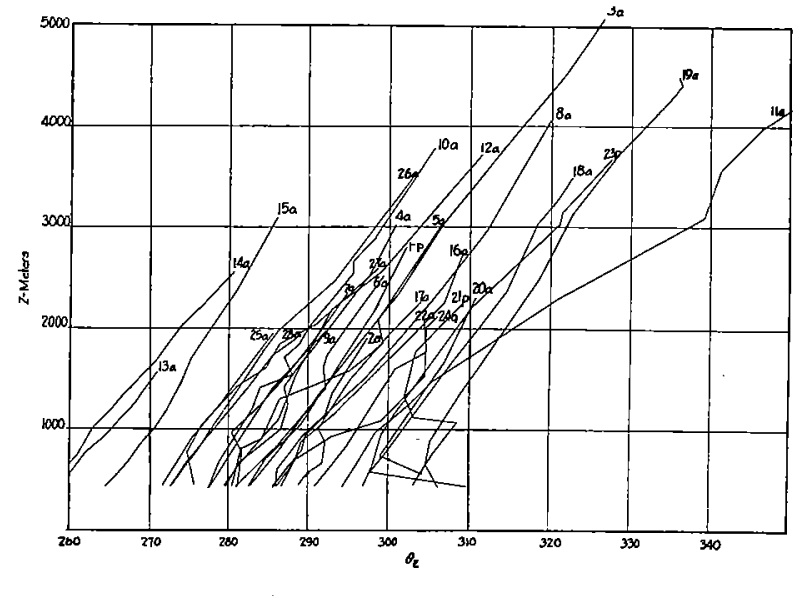

ELLENDALE, FEBRUARY, 1930

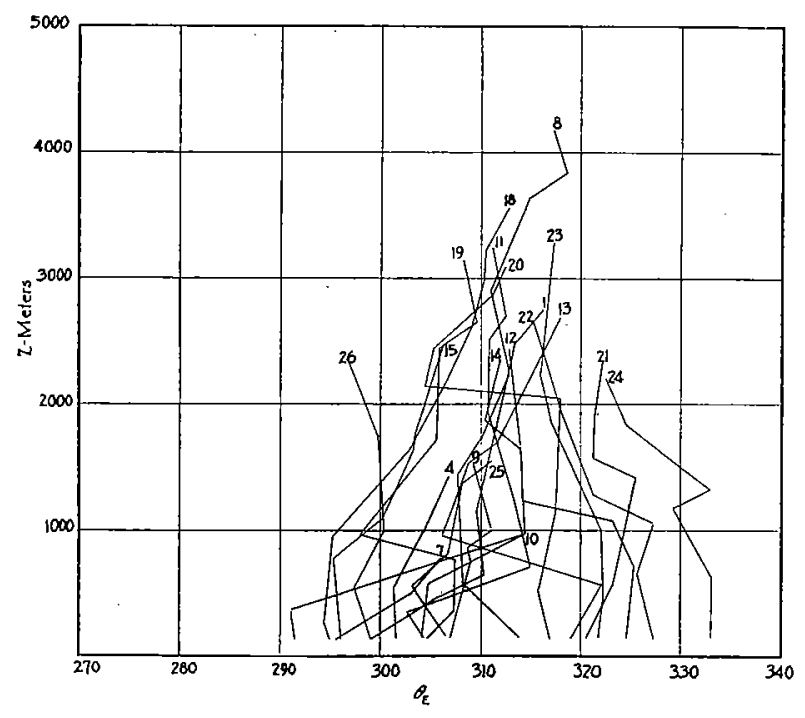

GROESBECK, FEBRUARY, 1930

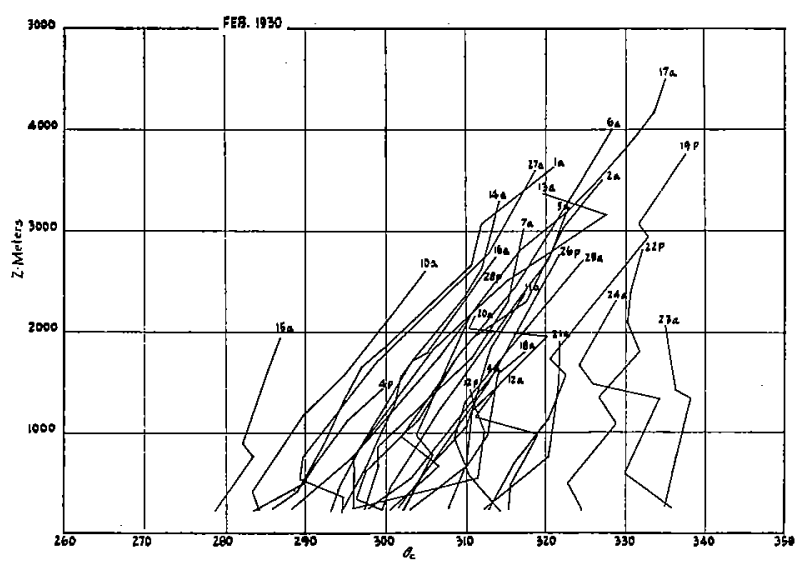

BROKEN ARROW, FEBRUARY, 1930

PLATE XI 